\title{
Reconstrução da Chave Secreta do RSA Multi-primo
}

\author{
Reynaldo Cáceres Villena
}

\author{
DissertaÇÃo APRESEnTADA \\ AO \\ Instituto de Matemática e Estatística \\ DA \\ Universidade DE SÃo PAUlo \\ PARA \\ OBTENÇÃO DO TÍTULO \\ $\mathrm{DE}$ \\ Mestre em CiÊnCIAS
}

Programa: Ciências da Computação

Orientador: Prof. Dr. Routo Terada

Durante o desenvolvimento deste trabalho

o autor recebeu auxílio financeiro da CAPES

São Paulo, Dezembro de 2013 


\section{Reconstrução da Chave Secreta do RSA Multi-primo}

Esta versão da dissertação contém as correções e alterações sugeridas pela Comissão Julgadora durante a defesa da versão original do trabalho, realizada em 23/09/2013. Uma cópia da versão original está disponível no

Instituto de Matemática e Estatística da Universidade de São Paulo.

Comissão Julgadora:

- Prof. Dr. Routo Terada (orientador) - IME-USP

- Prof. Dr. Marco Dimas Gubitoso - IME-USP

- Prof. Dr. Geraldo Lino de Campos - EP-USP 


\section{Agradecimentos}

Ao meu orientador Prof. Dr. Routo Terada que me introduziu à fascinante ciência da criptologia e me auxílio na elaboração deste trabalho.

Aos meus amigos do laboratório de segurança de dados do IME-USP. Dentre eles agradeço especialmente a Denise H. Goya e a Rafael Will M. de Araujo pelas observações importantes no decorrer do desenvolvimento desta dissertação.

A toda minha família; principalmente a meus pais Guillermo C. Nieto e Petronila V. Hinojosa pela ajuda financeira, intelectual e emocional.

À CAPES pelo suporte financeiro. 


\section{Resumo}

Em 2009, N. Heninger e H. Shacham apresentaram um algoritmo de reconstrução que permite recuperar a chave secreta $s k$ do criptossistema RSA básico em tempo polinomial tendo em forma aleatória $27 \%$ dos seus bits. Sabemos que podemos obter uma versão com erros (bits modificados) da chave secreta RSA graças aos ataques cold boot. O algoritmo apresentado por Heninger-Shacham corrige esses erros fazendo uso das relações matemáticas que existe entre as chaves pública e secreta do criptossistema RSA básico. O objetivo deste trabalho é estudar esse algoritmo para implementar e analisar seu análogo para o criptossistema RSA multi-primo. Os resultados obtidos mostram que para reconstruir a chave secreta $s k$ do criptossistema RSA $u$-primos é preciso ter uma fração de bits corretos maior a $2-2^{\frac{u+2}{2 u+1}}$, mostrando assim que a segurança oferecida pelo criptossistema RSA multi-primo $(u \geq 3)$ é maior com relação ao criptossistema $\operatorname{RSA}$ básico $(u=2)$.

Palavras-chave: Reconstrução da chave secreta, criptossistema RSA multi-primo, Ataques ColdBoot. 


\section{Abstract}

In 2009, N. Heninger and H. Shacham presented an algoritm for reconstructing the secret key $s k$ of the basic RSA cryptosystem in polynomial time with a fraction of random bits greater or equal to 0.27 of its bits. We know that secret key with errors $\tilde{s k}$ can be obtained from DRAM using cold-boot attacks. The Heninger and Shacham's algorithm fixes these errors using the redundancy of secret and public key of basic RSA cryptosystem. In this work, the topic is to study this algoritm to implement and analyze its analogous for the multi-prime RSA cryptosystem. Our obtained results show the secret key $s k$ of multi-prime RSA cryptosystem can be reconstructed having a fraction equal or greater than $2-2^{\frac{u+2}{2 u+1}}$ of random bits. therefore the security of multi-prime RSA cryptosystem $(u \geq 3)$ is greater than basic RSA cryptosystem $(u=2)$.

Keywords: Secret key reconstructing, Multi-prime RSA cryptosystem, Cold boot attacks. 


\section{Sumário}

Lista de Abreviaturas $\quad$ xi

Notação e Lista de Símbolos $\quad$ xiii

1 Introdução $\quad 1$

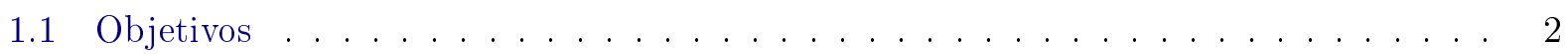

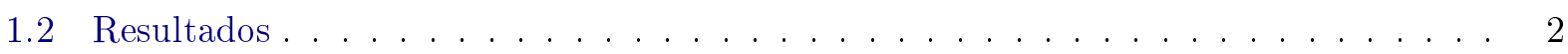

1.3 Organização do Trabalho . . . . . . . . . . . . . . . . . . . . . 2

2 Conceitos $\quad 3$

2.1 Criptografia e Criptografia de Chave Pública . . . . . . . . . . . . . . . 3

2.1 .1 Criptografia de Chave Pública . . . . . . . . . . . . . . . . . . 3

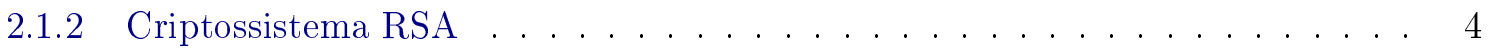

2.1.3 Método de Quisquater-Couvreur . . . . . . . . . . . . . . . . . 5

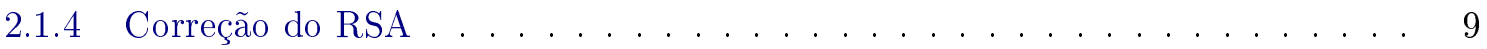

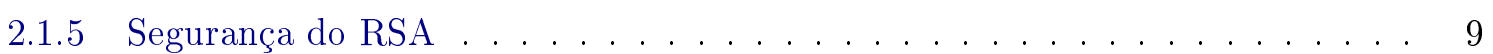

2.2 Public Key Criptography Standard - PKCS . . . . . . . . . . . . . . . . 10

$2.2 .1 \quad \mathrm{PKCS} \# 1 \ldots \ldots \ldots$

2.3 Ataques Side-Channel . . . . . . . . . . . . . . . . . . 11

2.3.1 Ataques Cold-boot . . . . . . . . . . . . . . . 12

2.3.2 Identificação de Chaves RSA na Memória . . . . . . . . . . . . . . . . . 13

3 Algoritmo de Reconstrução da Chave Secreta do Criptossistema RSA 15

3.1 Cálculo de Variáveis Auxiliares . . . . . . . . . . . . . . . . . . . . . 16

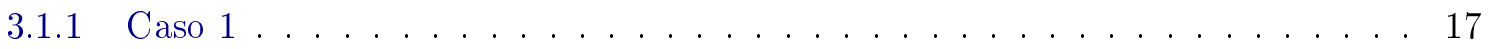

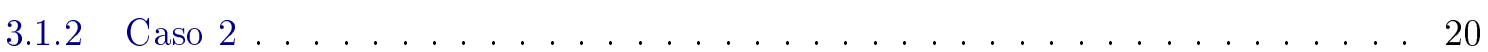

3.2 Correção de Alguns Bits . . . . . . . . . . . . . . . . . . . . . . . 24

3.3 Lema de Hensel - Geração das equações . . . . . . . . . . . . . . . . . . . . . 25

3.4 Algoritmo e seu comportamento . . . . . . . . . . . . . . . . . 28

3.4.1 Complexidade do Algoritmo . . . . . . . . . . . . . . . . . . . . . . 32

3.4 .2 Alguns Resultados . . . . . . . . . . . . . . . . . . . . . 48

4 Implementação e Resultados $\quad 51$

4.1 Implementação . . . . . . . . . . . . . . . . . . . . . . 51

4.2 Resultados para Chaves de 2048 Bits . . . . . . . . . . . . . . . . . . . 52

4.2.1 Resultados dos Experimentos do Criptossistema RSA Básico . . . . . . . . . . 52 
4.2.2 Resultados dos Experimentos do Criptossistema RSA 3-primos . . . . . . . 53

4.2.3 Resultados dos Experimentos do Criptossistema RSA 4-primos . . . . . . . . 53

4.2.4 Comparações . . . . . . . . . . . . . . . . . . . . . . . . . 54

4.3 Experimentos Extras ............................55

4.3.1 Experimentos para chaves de 3072 bits . . . . . . . . . . . . . . . . . 55

4.3.2 Experimentos para chaves de 4096 bits . . . . . . . . . . . . . . . . 55

5 Conclusões $\quad \mathbf{5 9}$

5.1 Conclusões ............................... 59

5.2 Recomendações . . . . . . . . . . . . . . . . . . . . . . . 61

5.3 Trabalhos Futuros e Problemas Abertos . . . . . . . . . . . . . . . . 61

A Teoria de Números $\quad \mathbf{6 3}$

A.1 Divisibilidade e Congruências . . . . . . . . . . . . . . . . . . . . . . . 63

A.1.1 Divisibilidade ......................... 63

A.1.2 Congruência ..............................63

A.1.3 Algoritmo de Euclides Estendido . . . . . . . . . . . . . . . . . . . . . 64

A.1.4 Inversa Multiplicativa Módulo . . . . . . . . . . . . . . . . . . . . . . . . . . . 64

A.1.5 Grupo e Anel . . . . . . . . . . . . . . . . . . . . . . . . 65

A.1.6 A Função Euler e o Teorema de Euler-Fermat . . . . . . . . . . . . . . . . . . 65

A.1.7 Polinômios . . . . . . . . . . . . . . . . . . . . . . . 66

A.2 Equações Módulo $m$. . . . . . . . . . . . . . . . . . . . 67

A.2.1 Equações Lineares Módulo $m$. . . . . . . . . . . . . . . . . . . . . . . . 67

A.2.2 Congruências de Grau 2 . . . . . . . . . . . . . . . . . . . . . . . . 68

A.3 Conceitos Extras . . . . . . . . . . . . . . . . . . . . . . . . . . 69

$\begin{array}{ll}\text { B Probabilidade } & 71\end{array}$

B.1 Experimentos Aleatórios .......................... 71

B.1.1 Introdução . . . . . . . . . . . . . . . . . . . . . . . . . 71

B.1.2 Espaço Amostral e Eventos . . . . . . . . . . . . . . . . . . . . . 71

B.1.3 Probabilidades ......................... 72

$\begin{array}{ll}\text { C Estatística } & 75\end{array}$

C.1 Variável Aleatória . . . . . . . . . . . . . . . . . . . . . . . . . 75

C.1.1 Variável Aleatória ........................... 75

C.2 Função de Probabilidade de uma Variável Aleatória . . . . . . . . . . . . . . . . . . 75

C.3 Distribuição de Probabilidade . . . . . . . . . . . . . . . . . . . 76

C.3.1 Valor Esperado ........................... 76

C.3.2 Variância .............................. 76

C.3.3 Desigualdade de Chebyshev . . . . . . . . . . . . . . . . . . . 76

C.3.4 Momentos ........................... 77

C.4 Distribuição Conjunta de Duas V.A. . . . . . . . . . . . . . . . . . . . . . . 77

C.4.1 Covariância .......................... 77

C.5 Coeficiente de Correlação de Pearson . . . . . . . . . . . . . . . . . . . . . 77

C.6 Funções Geratrizes .............................. 78 
C.6.1 Funções Geratrizes de Probabilidade . . . . . . . . . . . . . . . . . 78 


\section{Lista de Abreviaturas}

RSA Sistema Criptográfico desenvolvido por Rivest, Shamir e Adelman.

MIT Instituto Tecnológico de Massachusetts (Massachusetts Institute of Technology).

NP Classe de complexidade polinomial em modelo não determinístico (Non-Deterministic Polynomial time).

NFS É o algoritmo mais eficiente para fatorar inteiros de mais de 100 dígitos (Number Field Sieve).

PKCS Padrão de Criptografia de Chave Pública (Public Key Criptography Standards.)

TCR Teorema Chinês do Resto.

DRAM Memória de acesso aleatório dinámica (Dynamic Random-Access Memory).

MSB Bits mais significativos (Most Significant Bits).

LSB Bits menos significativos (Least Significant Bits).

ECM Método de curvas elípticas usado para calcular fatores primos de um inteiro composto. (Elliptic curve method).

MDC Máximo Divisor Comum. 


\section{Notação e Lista de Símbolos}

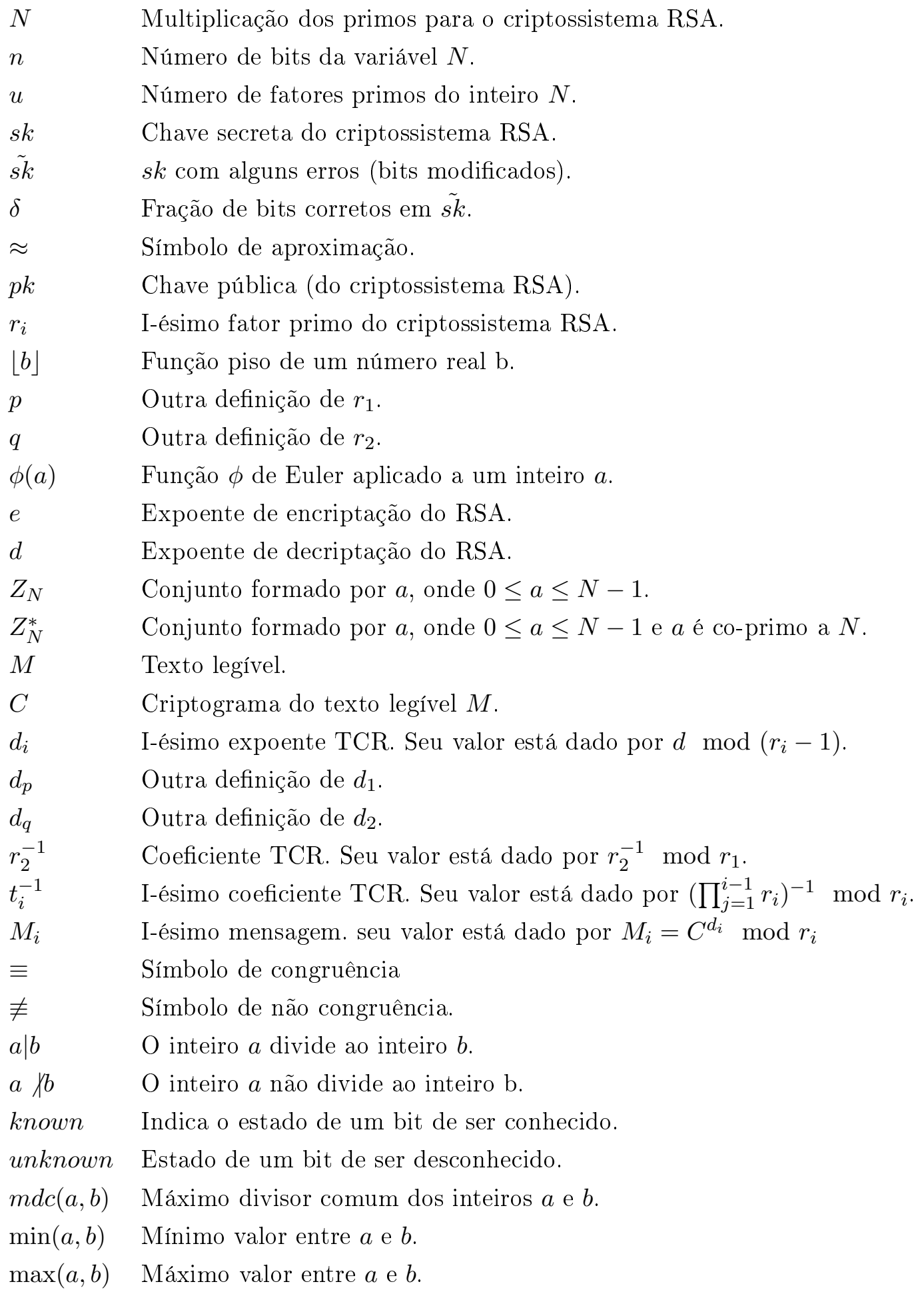




\section{Lista de Figuras}

2.1 Chave secreta RSA em ASN.1 (Fonte:PKCS \#1) . . . . . . . . . . . . . . . . 11

2.2 Desaparecimento gradual de dados em um chip de memória RAM (Fonte: Center for Information Technology at Princeton University - 2009) . . . . . . . . . . . . 12

2.3 Propriedade de remanescência da memória . . . . . . . . . . . . . . . . . 13

2.4 Codificação BER da chave secreta RSA. A parte sombreada mostra a cadeia 02-0100-02. (Fonte: Center for Information Technology at Princeton University) . . . . . . 14

3.1 Representação do comportamento do algoritmo de reconstrução onde as circunferências: simples são as raízes incorretas, as duplas são as raízes boas e por último temos a circunferência dupla cinza, a qual é a representação da raiz correta. . . . . . . . . . 31

3.2 Representação dos momentos de ordem 1 e 2 da variável discreta $G$, onde podemos observar que ambos momentos são iguais. . . . . . . . . . . . . 36

3.3 Representação dos momentos de ordem 1 e 2 da variável discreta $B$, onde podemos observar ambos momentos. . . . . . . . . . . . . . . . . . . . 40

4.1 Comparação das médias da quantidade de raízes analisadas para chaves de 2048 bits. 54

4.2 Comparação das médias da quantidade de raízes analisadas para chaves de 3072 bits. 56

4.3 Comparação das médias da quantidade de raízes analisadas para chaves de 4096 bits. 56 


\section{Lista de Tabelas}

2.1 Máximo número de primos permitidos em um módulo $N$ (Fonte: Compaq Computer Corporation) . . . . . . . . . . . . . . . . . . 10

3.1 Número de raízes geradas a partir de uma raiz boa. . . . . . . . . . . . . . . . 33

3.2 Número de raízes incorretas geradas a partir de uma raiz boa. . . . . . . . . . . . . 33

3.3 Estado do bit $r_{i}[j]$ com relação ao estado do bit $d_{i}\left[j+\tau\left(k_{i}\right)\right]$ na raiz boa. $\quad \ldots \ldots . .34$

3.4 Número de raízes incorretas geradas a partir de uma raiz incorreta. . . . . . . . . . . 37

3.5 Estado do bit $r_{i}[j]$ com relação ao estado do bit $d_{i}\left[j+\tau\left(k_{i}\right)\right]$ na raiz incorreta. . . . . 38

4.1 Resultados dos experimentos da Reconstrução da Chave Secreta RSA Básico $(u=2)$. 52

4.2 Resultados dos experimentos da Reconstrução da Chave Secreta RSA 3-primos. . . . 53

4.3 Resultados dos experimentos da Reconstrução da Chave Secreta RSA 4-primos. . . . 54

4.4 Resultados dos experimentos da Reconstrução da Chave Secreta RSA módulo 3072. . 55

4.5 Resultados dos experimentos da Reconstrução da Chave Secreta RSA módulo 4096. . 57

5.1 Comparação entre os criptossistemas RSA básico, 3-primos e 4-primos. . . . . . . . 60

5.2 Comparação entre os criptossistemas RSA multi-potência, básico e multi-primo. . . . 60

5.3 Número de raízes incorretas geradas a partir de uma raiz incorreta quando temos só uma porcentagem de bits conhecidos de $d \ldots \ldots \ldots \ldots$. . . . . . . 61 


\section{Capítulo 1}

\section{Introdução}

O RSA é um dos criptossistemas de chave pública mais usado e implementado [Pai03], seu nome é derivado das iniciais dos seus criadores: Ron (R)ivest, Adi (S)hamir e Len (A)dleman. Este criptossistema foi criado em agosto de 1977 no MIT $^{1}$ e publicado em fevereiro de 1978 [RSA78]. Desde sua publicação, nenhum ataque conseguiu quebrá-lo, portanto não foi preciso mudar sua estrutura. No entanto, na literatura cientifica existem pesquisas sobre casos onde o RSA é inseguro, mas isso é devido ao uso inadequado do mesmo.

A segurança do criptossistema RSA está baseada no problema de fatoração de um número inteiro (o qual é um problema NP) [Feo], no RSA o inteiro a fatorar é conhecido como módulo $N$ e é o resultado da multiplicação de $u$ primos aleatórios grandes do mesmo tamanho. Quando temos o valor $u=2$ é denominado como criptossistema RSA básico e para $u \geq 3$ é chamado de criptossistema RSA multi-primo. O algoritmo mais rápido até a data (2013) para a solução de fatoração de inteiros é conhecido como NFS (Number Field Sieve), o qual estabeleceu um novo recorde em fatorar um módulo $N$ de 768 bits (232 decimais) do criptossistema RSA básico em dezembro do 2009 [Con]. Observando a dificuldade de fatorar um inteiro surgiu a ideia de dar solução a esse problema fazendo uso de algum tipo de informação extra (neste caso, bits dos fatores primos de $N$ ou outros dados) que seria fornecida por um oraculo.

Em criptografia, um oráculo é um programa que responde perguntas com uma resposta booleana (um SIM ou um NÃO). Por exemplo, podemos perguntar "qual é o i-ésimo bit do menor fator de $N$ ?". No caso trivial para o módulo $N$ do criptosistema RSA Básico, só precisamos de $n / 2$ perguntas para fatorar $N$, onde $n=\lg N$ é o número de bits de $N$ [Mau92]. Com esse novo conceito surgiu uma nova linha de pesquisa assistida por um oráculo que procura minimizar o número de perguntas feitas ao oráculo. Correspondente a essa linha de pesquisa tem-se vários resultados eficientes onde a fatoração do modulo $N$ do criptossistema RSA básico é possível em tempo polinomial tendo:

- os $n / 4$ bits menos significativos (LSB) ou mais significativos (MSB) de um dos seus fatores primos $p$ [Cop97].

- os $n / 4$ LSB do expoente de decriptação $d$ [BDF98].

- os $n / 4$ LSB de expoente de decriptação $d_{p}$ [BDF98].

- um máximo de $\log \log (N)$ blocos desconhecidos e uma fração $\ln (2)=0.70$ de bits conhecidos de $p$ [HM08].

O algoritmo de reconstrução da chave secreta $s k$ do criptossistema RSA básico de Heninger e Shacham [HS09] está baseado em ataques assistidos por um oráculo, mas a diferença com os ataques antes mencionados é que o atacante não tem controle sobre as posições dos bits, ou seja, o atacante só recebe bits aleatórios. Os resultados de Heninger e Shacham mostram que é possível a reconstrução da chave secreta $s k$ do criptossistema RSA básico em tempo polinomial com uma grande probabilidade tendo uma fração $\delta$ maior ou igual a 0.27 de bits corretos em $\tilde{s k}^{2}$.

\footnotetext{
${ }^{1}$ Instituto Tecnológico de Massachusetts

${ }^{2}$ Estrutura de dados que contem alguns bits corretos de $s k$
} 


\subsection{Objetivos}

O objetivo principal desse projeto é a implementação de um algoritmo de reconstrução da chave secreta para o criptossistema RSA multi-primo $(u \geq 3)$, baseado no algoritmo de reconstrução para o criptossistema RSA básico $(u=2)$ proposto por Heninger e Shacham.

Como objetivos específicos deste projeto estão:

1. Estudo e análise dos criptossistemas RSA básico e RSA multi-primo.

2. Estudo e Implementação do algoritmo de reconstrução da chave secreta para o criptossistema RSA básico.

3. Estudo das relações que existem entre os bits das variáveis da chave secreta $s k$ dos criptossistemas RSA básico e RSA multi-primo.

4. Implementação e análise do algoritmo de reconstrução da chave secreta para o criptossistema RSA multi-primo.

\subsection{Resultados}

1. Para o criptossistema RSA básico ou multi-primo (onde seu módulo $N$ está dado por $N=$ $\prod_{i=1}^{u} r_{i}$ para $u \geq 2$ ) foi determinado que é preciso ter uma fração $\delta$ de bits corretos maior que $2-2^{\frac{u+2}{2 u+1}}$ em $\tilde{s k}$ para poder reconstruir a chave secreta $s k$ em tempo polinomial $O\left(n^{2}\right)$ com uma probabilidade maior que $1-\frac{1}{n^{2}}$. Esse resultado nos permite dizer que a reconstrução de uma chave secreta $s k$ para o criptossistema:

- RSA básico é preciso ter uma fração $\delta \geq 2-2^{\frac{4}{5}} \approx 0.27$

- RSA 3-primos é preciso ter uma fração $\delta \geq 2-2^{\frac{5}{7}} \approx 0.37$

- RSA 4-primos é preciso ter uma fração $\delta \geq 2-2^{\frac{6}{9}} \approx 0.42$

de bits corretos de $s k$.

2. Com o resultado acima foi comprovado que o criptossistema RSA multi-primo oferece mais segurança com relação ao criptossistema RSA básico.

$$
\delta>2-2^{\frac{u+2}{2 u+1}}>2-2^{\frac{4}{5}} \text { para } u \geq 3
$$

3. A reconstrução de uma chave secreta $s k$ do criptossistema RSA básico ou multi-primo sempre é feita em tempo polinomial quando tem se uma fração $\delta$ de bits maior ou igual a 0.59 .

$$
\delta>\lim _{u \rightarrow \infty} 2-2^{\frac{u+2}{2 u+1}} \approx 0.5858
$$

\subsection{Organização do Trabalho}

No Capítulo 2, apresentamos os conceitos básicos de criptografia, criptossistema RSA, padrão de criptografia de chave pública PKCS (Public key cryptography standart) do RSA e ataques side channel. O Capítulo 3 é descrito o algoritmo de reconstrução da chave secreta sk contendo: os precálculos que devem ser feitos, o algoritmo e uma análise da sua complexidade. No Capítulo 4 são mostrados os resultados da implementação do algoritmo. Finalmente no Capítulo 5 encontram-se as conclusões desse trabalho.

Os conceitos básicos matemáticos, probabilísticos e estatísticos que são utilizados no percorrer do trabalho são apresentamos nos Apêndices A, B e C respectivamente desse trabalho. 


\section{Capítulo 2}

\section{Conceitos}

Para iniciar nosso estudo sobre o algoritmo de reconstrução de chaves secretas RSA de Heninger e Shacham precisamos relembrar algumas definições e conceitos de criptografia, criptossistema RSA e ataques cold-boot e assim formar o necessário para a compreensão desse algoritmo. Portanto este capítulo serve como base para todos os algoritmos e ideias apresentadas ao longo desse documento e deve ser consultado assim que alguma dúvida surgir.

\subsection{Criptografia e Criptografia de Chave Pública}

Em grego, kryptós significa secreto ou oculto e gráphein se refere a escrita, portanto podemos definir à criptografia como o estudo dos princípios e técnicas pelas quais a informação pode ser transformada da sua forma original para outra ilegível, de forma que possa ser conhecida apenas por seu destinatário (detentor da chave secreta), o que a torna difícil de ser lida por alguém não autorizado. Portanto, só o receptor da mensagem pode ler a informação com facilidade [Hoo05]. O método mais simples consiste em substituir uma letra pela seguinte no alfabeto, isto é, transladar o alfabeto uma casa para diante de forma circular. Uma codificação semelhante a esta foi utilizada por Júlio César afim de estabelecer uma comunicação com alguma segurança com as legiões em combate pela Europa, ficando assim conhecida como cifra ${ }^{1}$ de César. Além disso, é um dos primeiros métodos de codificação de que se tem notícia.

A cifra de César pertence a uma classe de algoritmos conhecidos como algoritmos de chave secreta (ou simétrica). A chave secreta no caso da cifra de César seria o número de posições deslocadas em relação ao início do alfabeto (no caso acima somente uma posição). Observando este método notamos que a chave deve ser previamente combinada entre o emissor e o receptor da mensagem através de um meio sigiloso, além disso, deve ser mantida em segredo para evitar que uma pessoa não autorizada consiga ler a mensagem.

\subsubsection{Criptografia de Chave Pública}

A ideia de criptossistemas de chave pública ou assimétricos foi proposto por Diffie e Hellman em 1976 [DH76]. Em um criptossistema de chave pública cada entidade $A$ tem uma chave pública $p k$ (distribuída a todos) e uma chave secreta correspondente $s k$ (só $A$ conhece este valor). Em criptossistemas seguros, calcular $s k$ dado $p k$ é computacionalmente inviável. A chave pública $p k$ define um processo de encriptação $E_{p k}$, enquanto a chave secreta está associada a um processo de decriptação $D_{s k}$. Se qualquer entidade $B$ deseja enviar uma mensagem $M$ para $A, B$ deve obter uma cópia da chave pública $p k$ de $A$ e aplicar o processo de encriptação para obter o criptograma $C=E_{p k}(M)$, e enviá-lo para $A$. Para decriptar $C, A$ aplica o processo de decriptação usando sua chave secreta $s k$ para obter a mensagem original $M=D_{s k}(C)$.

Como cada entidade $A$ tem uma chave pública $p k_{A}$ e esta é distribuída de forma pública o problema de distribuição de chaves não existe nos criptossistemas assimétricos. Além de resolver

\footnotetext{
${ }^{1}$ Criptossistemas elementares são chamados de cifras
} 
esse problema, a criptografia de chave pública dá solução a outros problemas como:

1. Autenticação de Destino.- Esconder informações sigilosas das pessoas que controlam as linhas de comunicação e os computadores intermediários (provedores, roteadores) garantindo que o verdadeiro destinatário seja a única pessoa que consiga ler a informação enviada.

2. Autenticação de Origem.- Evitar que um terceiro falsifique a identidade do emissor enviando informação para o destinatário, em outras palavras, o destinatário deve ter certeza de que foi o verdadeiro emissor que envio a informação.

3. Integridade da Informação.- Evitar que um terceiro leia e altere a informação sem ser detectado, em outras palavras, o destinatário sabe se a mensagem foi alterada na linha de comunicação.

\subsubsection{Criptossistema RSA}

O criptossistema RSA foi inventado por R.Rivest, A. Shamir e L.Adleman em 1987 [RSA78] e é considerado como uns dos criptossistemas mais seguros já que nenhum ataque cripto-analítico conseguiu quebrá-lo até hoje (2013).

O criptossistema RSA está dividido em três algoritmos os quais são explicados embaixo. Denotaremos o emissor ou remetente da mensagem como Beto e o receptor ou destinatário como Alice. Existe também um terceiro ator mal-intencionado chamado Carlos que deseja ler a informação enviada na mensagem.

1. Geração de Chaves.- $O$ algoritmo de geração de chaves tem um parâmetro de entrada $n$, o qual indica o número de bits (parâmetro de segurança) de $N$. Cada usuário Alice deve obter aleatoriamente $u$ números primos $r_{1}, r_{2}, \ldots, r_{u-1}$ e $r_{u}$ de tamanho $\left\lfloor\frac{n}{u}\right\rfloor$ bits calculados por algum algoritmo determinístico ou probabilístico para calcular o inteiro $N=\prod_{i=1}^{u} r_{i}$. A seguir, determinamos um número inteiro e co-coprimo a $\phi(N)=\prod_{i=1}^{u}\left(r_{i}-1\right)$. Esse valor $e$ é conhecido como o expoente de encriptação (comumente fixado com o valor de $e=2^{16}+1=65537$ ). A chave pública de Alice é dada por $\langle N, e\rangle$ e a chave secreta é dada por $\langle N, d\rangle$ onde ambas chaves satisfazem a congruência $e d \equiv 1 \bmod \phi(N)$. O valor $d$ pode ser calculado com o algoritmo de Euclides-Estendido (veja o Apêndice A.1.3).

2. Encriptação.- Para Beto enviar uma mensagem $X$ para Alice, ele deve formatar $X$ segundo o padrão PKCS $\# 1^{2}$ [JK03] e obter um inteiro $M$ pertencente ao grupo $\mathbb{Z}_{N}$. Em seguida Beto obtém a chave pública de Alice $p k_{A}=\langle N, e\rangle$ e calcula o criptograma $C$ fazendo

$$
C \leftarrow M^{e} \bmod N
$$

para depois $C$ ser enviada para Alice.

3. Decriptação.- Para Alice poder ler a mensagem encriptada $C$ enviada por Beto, Alice utiliza a sua chave secreta $s k_{A}=\langle N, d\rangle$ para calcular

$$
M \leftarrow C^{d} \bmod N .
$$

A seguir, podemos aplicar o processo inverso da formatação para obter o valor da mensagem original $X$ a partir de $M$.

Quando temos $u=2$ então o criptossistema é conhecido como RSA básico. Para valores $u \geq 3$ é chamado de criptossistema RSA multi-primo ou $u$-primos.

\footnotetext{
${ }^{2}$ Padrão de criptossistemas de chave pública específico do criptossistema RSA
} 


\section{Exemplo Numérico de um Criptossistema RSA Básico}

\section{Geração de Chaves:}

$r_{1}=11, r_{2}=5$

$N=\prod_{i=1}^{2} r_{i}=55$

$\phi(N)=\prod_{i=1}^{2}\left(r_{i}-1\right)=40$

$e=3$

$d=e^{-1} \bmod \phi(N)=27$

Chave pública $\langle N, e\rangle=\langle 55,3\rangle$

Chave secreta $\langle N, d\rangle=\langle 55,27\rangle$

Encriptação: Para uma mensagem $M$ onde $0 \leq M \leq N-1$, neste caso $M=4$ temos

$$
\begin{aligned}
& C=M^{e} \bmod N \\
& C=4^{3} \quad \bmod 55 \\
& C=9
\end{aligned}
$$

Decriptação:

$$
\begin{aligned}
& M=C^{d} \bmod N \quad \text { Algoritmo (2.2) de decriptação } \\
& M=9^{27} \bmod 55 \\
& M=4
\end{aligned}
$$

\section{Exemplo Numérico de um Criptossistema RSA 3-primos}

\section{Geração de Chaves:}

$r_{1}=3, r_{2}=5, r_{3}=7$

$N=\prod_{i=1}^{3} r_{i}=105$

$\phi(N)=\prod_{i=1}^{3}\left(r_{i}-1\right)=48$

$e=5$

$d=e^{-1} \bmod \phi(N)=29$

Chave pública $\langle N, e\rangle=\langle 105,5\rangle$

Chave secreta $\langle N, d\rangle=\langle 105,29\rangle$

Encriptação: Para uma mensagem $M$ onde $0 \leq M \leq N-1$, neste caso $M=4$ temos

$$
\begin{aligned}
& C=M^{e} \quad \bmod N \\
& C=4^{5} \quad \bmod 105 \\
& C=79
\end{aligned}
$$

Algoritmo (2.1) de encriptação

\section{Decriptação:}

$$
\begin{aligned}
& M=C^{d} \bmod N \quad \text { Algoritmo (2.2) de decriptação } \\
& M=79^{29} \bmod 105 \\
& M=4
\end{aligned}
$$

\subsubsection{Método de Quisquater-Couvreur}

Em 1982 foi proposta uma nova técnica de decriptação para o criptossistema RSA básico por J-J. Quisquater e C. Couvreur [QC82]. Este método consegue obter $M$ de $C$ a partir de $M_{1}=C^{d}$ $\bmod r_{1}$ e $M_{2}=C^{d} \bmod r_{2}$ fazendo uso do TCR (veja o Apêndice A.2.1). No momento que fazemos o cálculo de $M_{1}$ e $M_{2}$ podemos reduzir o expoente de decifração $d$ módulo $r_{1}-1$ e $r_{2}-1$, ou seja, 
agora podemos calcular a mensagem $M$ com a seguinte equação

$$
M=\left(\left(M_{1}-M_{2}\right) r_{2}^{-1} \bmod r_{1}\right) r_{2}+M_{2}
$$

onde $d_{1}=d\left(\bmod r_{1}-1\right), d_{2}=d\left(\bmod r_{2}-1\right), M_{1}=C^{d_{1}}\left(\bmod r_{1}\right), M_{2}=C^{d_{2}}\left(\bmod r_{2}\right) \mathrm{e}$ $r_{2}^{-1}$ é $r_{2}^{-1}\left(\bmod r_{1}\right)$. Como podemos observar, o valor $d$ da chave secreta RSA não é usada mais, portanto, a chave secreta muda a $s k=\left\langle r_{1}, r_{2}, d_{1}, d_{2}, r_{2}^{-1}\right\rangle$. O tempo de execução da Fórmula (2.3) é até 4 vezes mais rápido do que a aplicação de $M \leftarrow C^{d} \bmod N$, a partir disso este método ficou muito popular. Esse método apresentado por J-J. Quisquater e C. Couvreur foi adaptado para o criptossistema RSA básico e multi-primo devido ao seu menor tempo no processo de decriptação com respeito ao Algoritmo (2.2).

Para o uso do método de Quisquater-Couvreur no criptossistema RSA básico e multi-primo a chave secreta está definida por

$$
s k=\left\langle r_{1}, r_{2}, d_{1}, d_{2}, r_{2}^{-1},\left\langle r_{3}, d_{3}, t_{3}\right\rangle, . .,\left\langle r_{u}, d_{u}, t_{u}\right\rangle\right\rangle
$$

onde as cinco primeiras variáveis seguem tendo a mesma definição e as variáveis das triplas estão definidas por

$$
\begin{aligned}
d_{i} & \equiv d & & \left(\bmod r_{i}-1\right) \\
t_{i} R_{i} & \equiv 1 & & \left(\bmod r_{i}\right)
\end{aligned}
$$

onde $R_{i}=\prod_{j=1}^{i-1} r_{j}$, para $3 \leq i \leq u$. O novo processo para decriptar o criptograma $C$ baseado no método Quisquater-Couvreur está descrito no Algoritmo 1 [JK03].

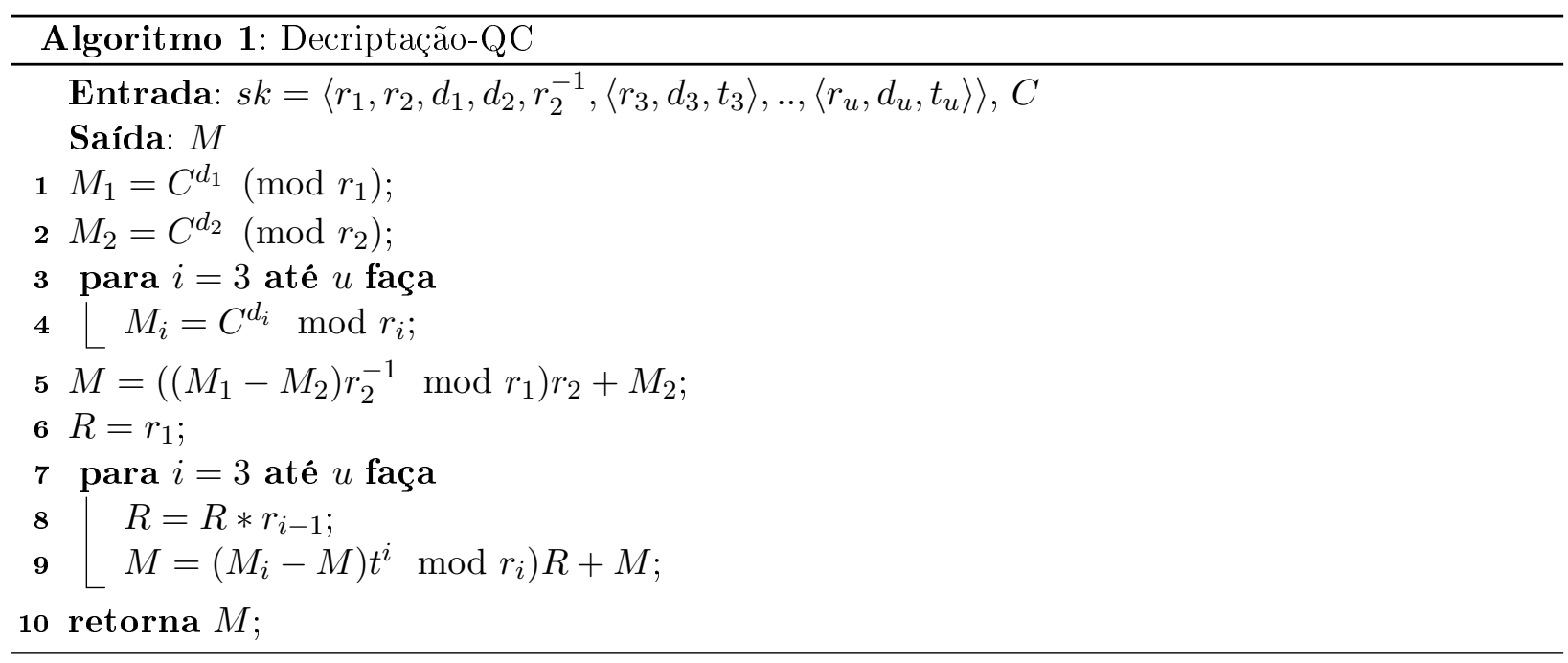


Exemplo Numérico do Método Quisquater-Couvreur para o Criptosistema RSA Básico

Geração de Chaves:

$r_{1}=11, r_{2}=5$

$N=\prod_{i=1}^{2} r_{i}=55$

$\phi(N)=\prod_{i=1}^{2}\left(r_{i}-1\right)=40$

$e=3$

$d=e^{-1} \bmod \phi(N)=27$

$d_{1}=d \bmod \left(r_{1}-1\right)=27 \bmod 10=7$

$d_{2}=d \bmod \left(r_{2}-1\right)=27 \bmod 4=3$

$r_{2}^{-1}=r_{2}^{-1} \bmod r_{1}=5^{-1} \bmod 11=9$

Chave pública $\langle N, e\rangle=\langle 55,3\rangle$

Chave secreta $\left\langle r_{1}, r_{2}, d_{1}, d_{2}, r_{2}^{-1}\right\rangle=\langle 11,5,7,3,9\rangle$

Encriptação: Para uma mensagem $M$ onde $0 \leq M \leq N-1$, neste caso $M=4$ temos

$$
\begin{aligned}
& C=M^{e} \bmod N \\
& \text { Algoritmo (2.1) de encriptação } \\
& C=4^{3} \quad \bmod 55 \\
& C=9
\end{aligned}
$$

Decriptação (usando o Algoritmo 1):

$$
\begin{aligned}
M_{1} & =C^{d_{1}} \quad \bmod r_{1} \\
M_{1} & =9^{7} \quad \bmod 11 \\
M_{1} & =4 \\
M_{2} & =C^{d_{2}} \bmod r_{2} \\
M_{2} & =27^{3} \quad \bmod 5 \\
M_{2} & =4 \\
M & =\left(\left(M_{1}-M_{2}\right) r_{2}^{-1} \bmod r_{1}\right) r_{2}+M_{2} \\
M & =((4-4) 9 \bmod 11) * 5+4 \\
M & =4
\end{aligned}
$$


Exemplo Numérico do Método Quisquater-Couvreur para o Criptosistema RSA 3primos

\section{Geração de Chaves:}

$$
\begin{aligned}
& r_{1}=3, r_{2}=5, r_{3}=7 \\
& N=\prod_{i=1}^{3} r_{i}=105 \\
& \phi(N)=\prod_{i=1}^{2}\left(r_{i}-1\right)=48 \\
& e=5 \\
& d=e^{-1} \bmod \phi(N)=29 \\
& d_{1}=d \bmod \left(r_{1}-1\right)=29 \bmod 2=1 \\
& d_{2}=d \bmod \left(r_{2}-1\right)=29 \bmod 4=1 \\
& d_{3}=d \bmod \left(r_{3}-1\right)=29 \bmod 6=5 \\
& r_{2}^{-1}=r_{2}^{-1} \bmod r_{1}=5^{-1} \bmod 3=2 \\
& R_{3}=r_{1} r_{2}=3 * 5=15 \\
& t_{3}=\left(R_{3}\right)^{-1} \bmod r_{3}=15^{-1} \bmod 7=1 \\
& \text { Chave pública }\langle N, e\rangle=\langle 105,5\rangle \\
& \text { Chave secreta }\left\langle p, q, d_{p}, d_{q}, q^{-1},\left(r_{3}, d_{3}, t_{3}\right)\right\rangle=\langle 3,5,1,1,2,\langle 7,5,1\rangle\rangle
\end{aligned}
$$

Encriptação: Para uma mensagem $M$ onde $0 \leq M \leq N-1$, neste caso $M=4$ temos

$$
\begin{aligned}
& C=M^{e} \bmod N \quad \text { Algoritmo (2.1) de encriptação } \\
& C=4^{5} \bmod 105 \\
& C=79
\end{aligned}
$$

\section{Decriptação (usando o Algoritmo 1):}

$$
\begin{aligned}
M_{1} & =C^{d_{1}} \quad \bmod r_{1} \\
M_{1} & =79^{1} \quad \bmod 3 \\
M_{1} & =1 \\
M_{2} & =C^{d_{2}} \quad \bmod r_{2} \\
M_{2} & =79^{1} \quad \bmod 5 \\
M_{2} & =4 \\
M_{3} & =C^{d_{3}} \quad \bmod r_{3} \\
M_{3} & =79^{5} \quad \bmod 7 \\
M_{3} & =4 \\
M & =\left(\left(M_{1}-M_{2}\right) r_{2}^{-1} \bmod r_{1}\right) r_{2}+M_{2} \\
M & =((1-4) 2 \quad \bmod 3) * 5+4 \\
M & =4 \\
R & =3 \\
\text { para } u=3 & \\
R & =r_{2} * R=3 * 5=15 \\
M & =\left(\left(M_{3}-M\right) t_{3}^{-1} \bmod r_{3}\right) R+M \\
M & =((4-4) 1 \quad \bmod 7) * 15+4 \\
M & =4
\end{aligned}
$$




\subsubsection{Correção do RSA}

A seguir para determinar o funcionamento do criptossistema RSA vamos provar que $\left(M^{e}\right)^{d}=M$ $\bmod N$, tendo $e d=1 \bmod \phi(N)$, o qual pode ser expressado como $e d=1+k \phi(N)$ onde $k$ é um inteiro positivo. A prova para $M \in \mathbb{Z}_{N}^{*}$ é descrita a seguir:

$$
\left(M^{e}\right)^{d}=M^{1+k \phi(N)} \bmod N
$$

Vamos lembrar pela definição de congruência $e d=1 \bmod \phi(N)$ é igual a $e d=1+k \phi(N)$. Continuando

$$
\begin{array}{rlrl}
\left(M^{e}\right)^{d} & =\left(M^{\phi(N)}\right)^{k} * M & \\
& =M \bmod N & & \\
& & \text { pelo Corolário } 1
\end{array}
$$

Provamos que o RSA funciona para todo $M \in \mathbb{Z}_{N}^{*}$, mas agora devemos provar que o RSA funciona para todo $M \in \mathbb{Z}_{N}$. Para isso, devemos provar que $\left(M^{e}\right)^{d}=M \bmod r_{i}$ para todo $1 \leq i \leq u$ (já que $N=\prod_{i=1}^{u} r_{i}$ onde $r_{1}, r_{2}, \ldots, r_{u}$ são primos distintos), e a seguir resolver o sistema com o TCR. Faremos a prova apenas para $r_{1}$ já que o calculo é análogo a $r_{i}$ para $2 \leq i \leq u$.

Temos que $e d=1 \bmod \phi(N)$ e portanto $e d=1+k\left(r_{1}-1\right) \prod_{i=2}^{u}\left(r_{i}-1\right)$ (veja a Definição 10$)$, portanto temos que:

$$
\begin{aligned}
\left(M^{e}\right)^{d} & =M^{1+k\left(r_{1}-1\right) \prod_{i=2}^{u}\left(r_{i}-1\right)} \bmod r_{1} \\
& =M\left(M^{\left(r_{1}-1\right)}\right)^{k \prod_{i=2}^{u}\left(r_{i}-1\right)} \bmod r_{1}
\end{aligned}
$$

Considerando que o $m d c\left(M, r_{1}\right)=1$, temos pelo teorema de Euler (veja o Teorema 2) que $M^{\phi\left(r_{1}\right)} \equiv 1 \bmod r_{1}$ (e como $r_{1}$ é primo temos que $\phi\left(r_{1}\right)=r_{1}-1$ assim podemos mostrar que:

$$
\begin{aligned}
\left(M^{e}\right)^{d} & =M(1)^{k \prod_{i=2}^{u}\left(r_{i}-1\right)} \bmod r_{1} \\
& =M \bmod r_{1}
\end{aligned}
$$

Da mesma forma podemos provar que $\left(M^{e}\right)^{d}=M \bmod r_{i}$ para $2 \leq i \leq u$. Em outra palavras, sabemos que $\left(M^{e}\right)^{d}=M \bmod r_{i}$ para $1 \leq i \leq u$ e pela equação TCR (veja A.6) concluímos que $\left(M^{e}\right)^{d}=M \bmod N$ para todo $M \in \mathbb{Z}_{N}$.

\subsubsection{Segurança do RSA}

O RSA como sistema criptográfico de chave pública deve cumprir que a recuperação da chave secreta a partir da chave pública tem que ser um problema computacionalmente inviável, ou seja, o cálculo de $d$ deve ser difícil apenas tendo os valores de $e$ e $N$. A única maneira para calcular $d$ é aplicando o algoritmo de Euclides-Estendido aos valores e e $\phi(N)$, mas para obter o valor de $\phi(N)$ precisamos saber os valores dos primos $r_{i}$ (para $1 \leq i \leq u$ ) ou simplesmente fatorar $N$. Portanto, a segurança do criptossistema RSA está baseada no problema da fatoração de inteiros o qual é computacionalmente inviável para primos grandes, mas podemos propor os seguintes ataques:

1. Cálculo de $\phi(N)$ sem fatorar $N$

2. Determinação de $d$ sem fatorar $N$ e sem calcular $\phi(N)$

3. Cálculo de um $d^{\prime}$ equivalente a $d$

Como é mostrado em [Ter00], realizar esses ataques é pelo menos tão caro computacionalmente quanto o melhor algoritmo para fatorar inteiros conhecido até a data em que este trabalho foi escrito (2013), portanto podemos concluir que o criptossistema RSA é seguro.

Sabemos que o algoritmo de fatoração de inteiros mais rápido e genérico é o NFS [Len01], onde seu tempo esperado heurístico para calcular um fator não-trivial do inteiro $N$ está dado por

$$
O\left(e^{1.923 \log ^{\frac{1}{3}} n \log ^{\frac{2}{3}} \log n}\right) .
$$


Observar-se que o tempo de execução do NFS só depende do número de bits do inteiro $N$, portanto não depende do valor de $u$, o qual indica que o tempo de execução é o mesmo para um $N=\prod_{i=1}^{u} r_{i}$ onde $u \geq 2$. O maior inteiro fatorado pelo algoritmo NFS foi de 232 dígitos (768 bits) realizado em 12 de dezembro do 2009 [Con].

O método de curvas elípticas ECM usado na fatoração pode calcular um fator primo do inteiro $N$. Seu tempo é menor em comparação ao NFS quando o fator primo a calcular tem um tamanho menor que $\sqrt{N}$. A Complexidade de tempo computacional do ECM é dada por

$$
O\left(\log ^{2} n e^{\sqrt{2} \log ^{\frac{1}{2}} \frac{n}{u} \log ^{\frac{1}{2}} \log \frac{n}{u}}\right)
$$

onde claramente podemos observar que sua complexidade é sub-exponencial ao tamanho do fator primo $\left(\frac{n}{u}\right)$ e polinomial com relação a $n$ [Len01]. Portanto podemos afirmar que o tempo de execução do ECM é decrementado a cada primo agregado ao módulo $N$. O maior fator encontrado pelo ECM foi de 75 dígitos em 2 de agosto de 2012 [Zim].

Como já foi mencionado, a segurança do criptossistema RSA está baseada no problema da fatoração do inteiro $N$. E com cada primo acrescentado a segurança relativa diminui, portanto para manter essa segurança, a quantidade de fatores primos de $N$ deve ser pequena. Na Tabela 2.1 temos os máximos valores estimados para o valor de $u$ considerando que o inteiro $N$ é seguro. Esta informação foi tomada de [Cor], e foi determinada pela análise conjunto dos tempos de execução do NFS e ECM.

\begin{tabular}{|c|c|c|c|c|c|}
\hline Número de bits de $N(\mathrm{n})$ & 1024 & 2048 & 3072 & 4096 & 8192 \\
\hline Máximo número de primos $(u)$ & 3 & 3 & 3 & 4 & 5 \\
\hline
\end{tabular}

Tabela 2.1: Máximo número de primos permitidos em um módulo $N$ (Fonte: Compaq Computer Corporation).

\subsection{Public Key Criptography Standard - PKCS}

O PKCS é um conjunto de padrões feitos pelos laboratórios $R S A^{3}$ (em consórcio com outras companhias Apple, Microsoft, Sun, Lotus, DEC, etc...) que contém especificações para acelerar a implementação e desenvolvimento dos algoritmos dos criptossistemas de chave pública [Lab91a].

Neste estudo vamos fazer uma análise do PKCS \#1 (padrão específico do criptossistema RSA) já que precisamos conhecer algumas especificações que depois serão pontos fracos para efetuar o ataque.

\subsubsection{PKCS \#1}

O PKCS \#1 é o primeiro da família de padrões PKCS, ele contém definições básicas e recomendações para a implementação do algoritmo RSA, também contém propriedades matemáticas da chave pública e secreta, as operações para encriptação de dados, assinatura digital e um esquema criptográfico seguro [JK03].

Para este trabalho vamos fazer um estudo do padrão PKCS \#1 versão 2.1 enfatizando na declaração da chave secreta. Segundo o padrão temos duas representações:

1. A primeira representação consiste no par $\langle N, d\rangle$ com os quais podemos aplicar o Algoritmo (2.2) para obter $M$.

2. A segunda representação consiste em uma quíntupla $\left\langle r_{1}, r_{2}, d_{1}, d_{2}, r_{2}{ }^{-1}\right\rangle$ e uma possível sequência de triplos $\left\langle r_{i}, d_{i}, t_{i}\right\rangle, i=3,4, \ldots, u$, uma tripla por cada primo extra que não está

\footnotetext{
${ }^{3}$ Empresa dedicada à criptografia e ao desenvolvimento de software de segurança
} 
incluso na quíntupla (se refere a uma possível implementação do criptossistema RSA multiprimo), onde os parâmetros são:

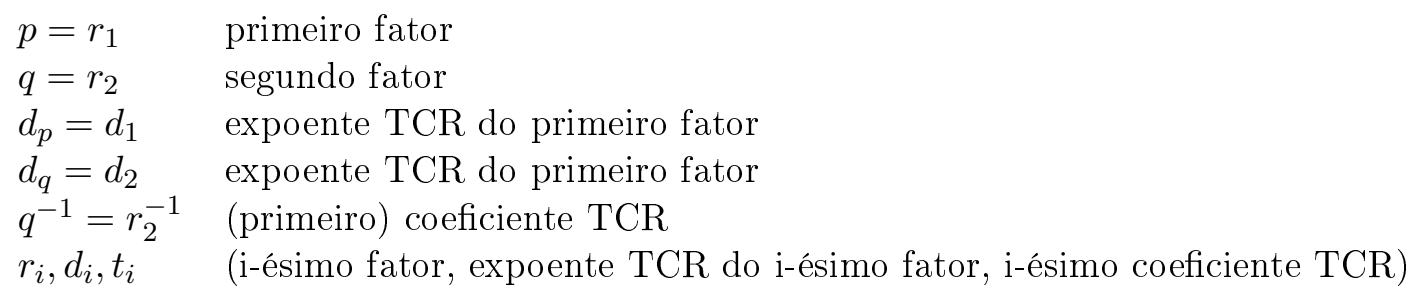

Com esta representação podemos aplicar o Algoritmo 1 baseado no método QuisquaterCouvreur (veja o Seção 2.1.3) para decriptar o criptograma $C$ em um tempo menor.

Usando qualquer das duas representações podemos fazer o processo de decriptação, portanto podemos usar qualquer das duas representações e ter o mesmo resultado final, mas segundo o PKCS \#1 a notação sintática abstrata um (ASN.1) ${ }^{4}$ da chave secreta deve ser representada por todos os parâmetros das duas representações da chave secreta conjuntamente com a chave pública (veja a Figura 2.1 para maior entendimento):

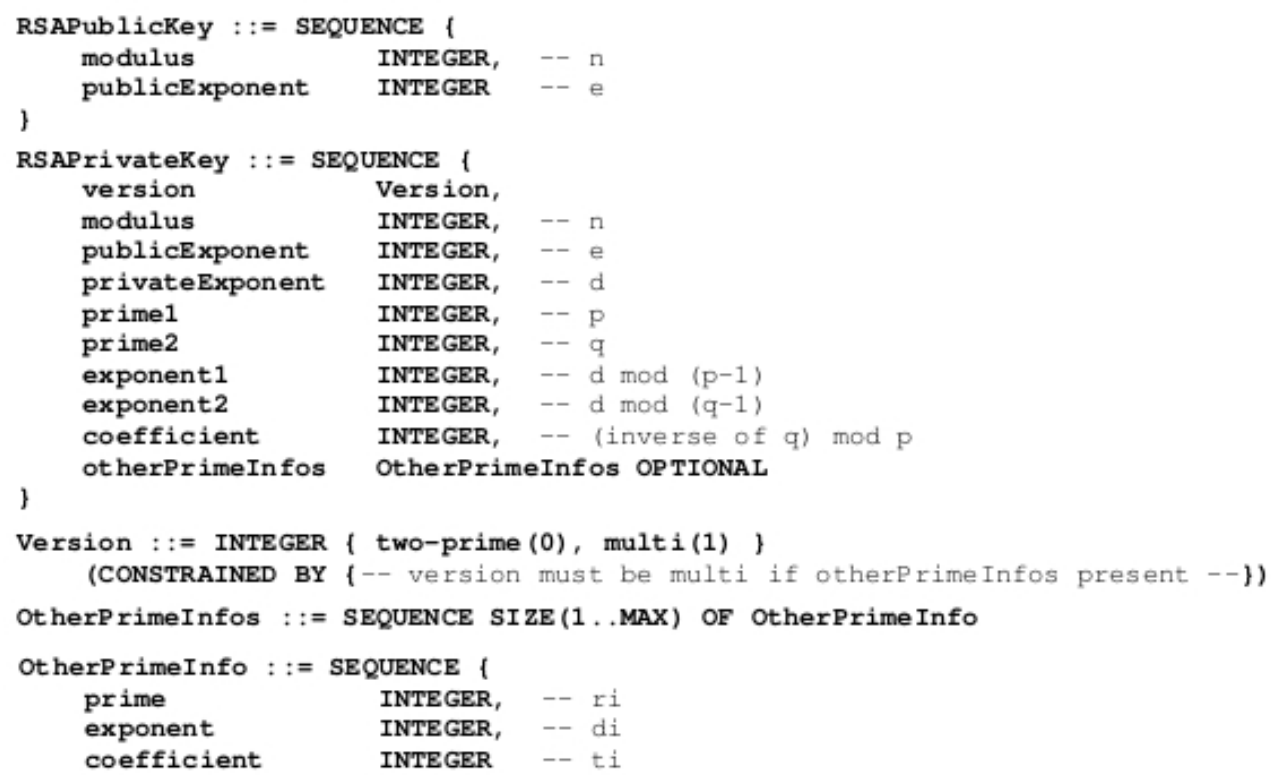

Figura 2.1: Chave secreta RSA em ASN.1 (Fonte:PKCS \#1)

Na Figura 2.1 a variável version é um valor booleano: 0 se o $\mathrm{N}$ for a multiplicação de dois primos e 1 se for de três ou mais primos. A variável otherPrimeInfos representa um vetor de dados do tipo OtherPrimeInfo onde esta estrutura está composta por uma tripla de inteiros (prime, expoent, coefficient) correspondente ao valores $r_{i}, d_{i}, t_{i}$ antes vistos. As outras variáveis têm o valor que representam ao lado.

\subsection{Ataques Side-Channel}

Para maior entendimento de ataques side-channel (ataques de canal secundário) primeiro vamos definir o que é a informação side-channel. A informação side-channel é toda informação que pode ser obtida do dispositivo onde nosso criptossistema foi implantado enquanto os processos de encriptação

\footnotetext{
${ }^{4}$ É um padrão de regras e estruturas para a representação, codificação, transmissão e decodificação de dados em telecomunicações e redes.
} 
e decriptação estão em operação. A informação side-channel não é nem a mensagem $M$ e nem o criptograma $C[\mathrm{BE} 03]$.

No passado, simplesmente considerava-se que o dispositivo de encriptação era uma unidade que tinha uma entrada legível $M$ para gerar a saída criptografada $C$ ou vice-versa (uma entrada cifrada, o criptograma $C$, para gerar a saída decifrada $M$ ). Com essa definição só tínhamos ataques baseados no conhecimento do criptograma (ataque por só texto ilegível), ou no conhecimento da mensagem e do seu criptograma (ataque por texto ilegível conhecido), ou podendo ter escolhido a mensagem a ser encriptado (ataque por texto legível escolhido) e demais variantes desses [Ter00]. Atualmente, sabemos que os dispositivos de encriptação podem ter entradas e saídas adicionais além da mensagem $M$ e do criptograma $C$.

Os dispositivos de encriptação produzem saídas enquanto o processo de encriptação ou decifração estão em operação como: informação de tempo de demora dos processos, consumo de eletricidade, radiação de vários tipos, sons produzidos e demais. Um tipo de entrada adicional poderia ser a variação intencional no voltagem da fonte de alimentação. Como observamos, para efetuar esses ataques devemos conhecer o funcionamento e propriedades do hardware. Na próxima seção é explicado um ataque que faz proveito de uma propriedade da memória DRAM para recuperar informações armazenadas nele, este ataque é conhecido como ataque cold-boot.

\subsubsection{Ataques Cold-boot}

O termo cold-boot (é também conhecido como hard-boot) se refere ao reiniciamento de um computador depois de ter sido cortada sua fonte de energia abruptamente. Já que o computador não foi desligado corretamente o sistema operacional não fez os processos correspondentes para o desligamento do computador, portanto isto pode causar perda ou corrupções nos dados que estavam sendo usados por algum processo nesse instante.

Com esse significado de cold-boot podemos definir que um ataque cold-boot é aquele onde o atacante tem acesso físico ao computador e é capaz de recuperar dados da memória DRAM/SRAM depois de ser aplicado um cold-boot ao computador. Esse ataque confia plenamente na propriedade de remanescência ou persistência da memória DRAM/SRAM o qual nós permite recuperar dados da memória que são conservados por um determinado tempo após a remoção da energia.

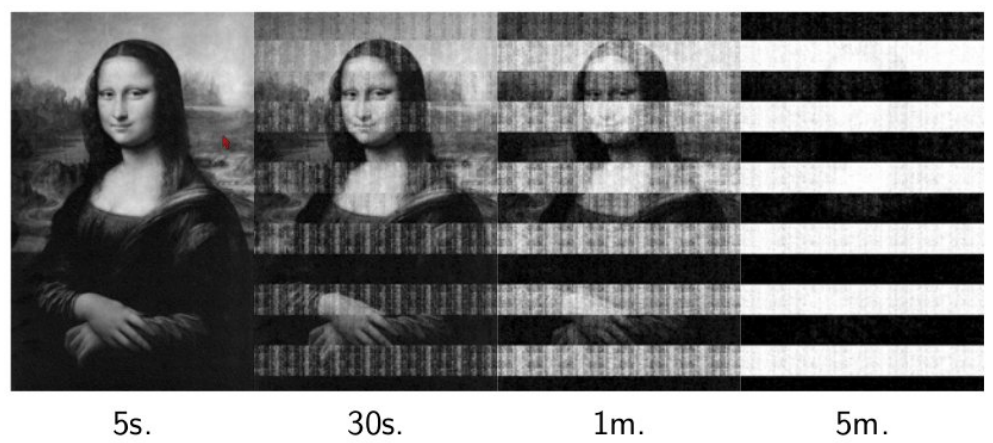

Figura 2.2: Desaparecimento gradual de dados em um chip de memória RAM (Fonte: Center for Information Technology at Princeton University - 2009)

A conservação dos bits na memória DRAM só dura alguns segundos, entre 2 a 35 segundos, a depender da marca e do modelo utilizado [HSH+ $\left.{ }^{+} 09\right]$. Vamos supor a efetuação desse ataque, depois de fazer o cold-boot no computador, no pior dos casos só temos 2 segundos para fazer uma leitura dos dados da memória, e ligar o computador pode demorar mais tempo do que isso. Portanto precisamos acrescentar esse tempo de conservação dos bits e é conhecido que as técnicas de esfriamento trabalham perfeitamente nesse campo [Sko02].

Com o computador ligado esfriamos o chip da memória, e efetuamos um cold-boot. Enquanto o computador está desligado a temperatura deve ser mantida no chip de memória até que o computa- 
dor volte a ser ligado. Esfriando o chip da memória com um canned air ${ }^{5}$ podemos conseguir uma temperatura de $-50^{\circ} \mathrm{C}$ e assim é possível conservar os bits por aproximadamente 60 segundos, se o chip é submerso em nitrogênio líquido podemos conservar a informação por mais de 60 minutos. Isto mostra que só é necessário um bom sistema de esfriamento para poder recuperar os dados depois de horas ou dias $\left[\mathrm{HSH}^{+} 09\right]$.

Como sabemos uma DRAM é um conjunto de capacitadores (cada bit é representado por um capacitador) que podem ter o estado 1 (com energia) ou estado 0 (sem energia). Depois da efetuação do cold-boot os capacitadores podem modificar sua carrega e, portanto, trocar seu estado dependendo aos blocos onde eles pertencem. Na memória DRAM existem blocos de bits onde o decaimento de bits acontece para o estado 1 ou estado 0 (veja na quarta imagem da Figura 2.2). Em um bloco de bits onde o decaimento é para o estado 0 temos que os capacitadores de estado 1 podem mudar para o estado 1 ou $0(1 \rightarrow 1$ ou $1 \rightarrow 0)$ enquanto os capacitadores de estado 0 não mudam $(0 \rightarrow$ 0 ), o contrário acontece no bloco de bits onde o decaimento tende para o estado 1. Os bits que não sofreram muda de estado após do cold-boot são conhecidos como bits remanescentes ou persistentes e os outros como bits deteriorados ou decaídos. Portanto podemos definir que uma imagem perfeita da memória é aquela que seus bits não sofreram trocas (veja na Figura 2.3), e uma imagem residual é aquela que sofreu modificações nos bits onde $\delta$, a fração dos bits corretos ou remanescentes, é dada por

$$
\delta=\frac{(1-\rho)}{2}
$$

onde $\rho$ é a fração de bits decaídos supondo que o número de bits decaídos para o estado 1 seja igual ao número de bits decaídos para o estado 0 [HS09].

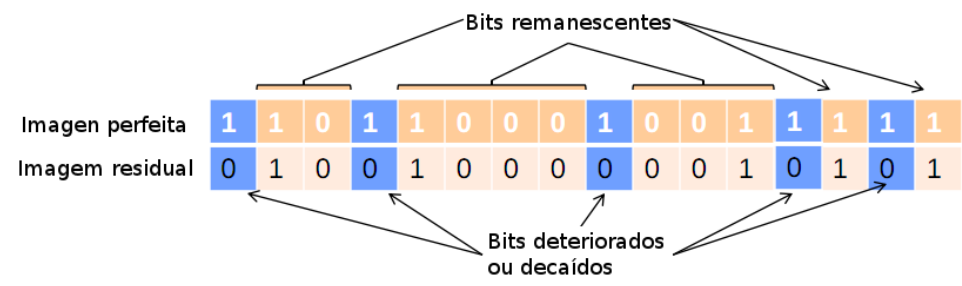

Figura 2.3: Propriedade de remanescência da memória

Para a obtenção uma imagem residual da memória DRAM não é preciso sistemas especiais, já que quando o sistema inicia o controlador da memória começa fazer o atualização da DRAM (lê e reescreve cada valor dos bits). Neste ponto os bits são fixos e qualquer programa sobre o sistema pode ler qualquer dado usando só instruções de acesso a memória. Existem várias formas de obter uma imagem residual da memória: uma seria fazer uma pequena aplicação $\mathrm{PXE}^{6} \mathrm{com}$ a única função de enviar o conteúdo da memória usando o protocolo $\mathrm{UDP}^{7}$, e a outra, podemos implementar um plugin para SYSLINUX bootloader ${ }^{8}$ o qual pode ser inicializado desde um dispositivo USB externo ou um disco rígido para salvar uma cópia do conteúdo da memória DRAM em uma partição particular designada do mesmo [Lab91b]. Nestes procedimentos devemos evitar a inicialização de sistemas grandes já que podem reescrever grandes partes da memória, ou seja, a inicialização de um sistema operacional completo seria muito destrutivo para o ataque.

\subsubsection{Identificação de Chaves RSA na Memória}

A extração de chaves de decifração de uma imagem da memória requer mecanismos de localização das chaves objetivo. Para o criptossistema RSA básico Um desses mecanismos seria testar

\footnotetext{
${ }^{5}$ Conhecido também com gas duster ou ar comprimido. É um produto usado para limpar equipamento eletrônico ou dispositivos sensíveis que não possam ser limpados com água.

${ }^{6}$ Ambiente para inicializar computadores usando a interface da placa de rede

${ }^{7}$ Protocolo simples da camada de transporte

${ }^{8}$ São gestores ligeiros para a inicialização de computadores no sistema operacional Linux
} 
todas as sequências de $n$ bits de nossa imagem e esperar se uma delas possa decriptar algum criptograma conhecido. Outra forma de encontrar seria pegar dois blocos seguidos de $n / 2$ e efetuar a multiplicação e comparar com $N$, se o resultado for igual então temos os valores dos primos $r_{1}$ e $r_{2}$. Também podemos calcular a metade superior dos bits de $d$ e procurá-los na imagem, depois disso basta pegar os bits inferiores de $d\left(\left[\mathrm{HSH}^{+} 09\right]\right.$ e [SS98]). Podemos ver que esses métodos demoram muito tempo, como mínimo para uma memória RAM de 1 GB precisamos fazer $2^{28}$ testes. A seguir, vamos explicar uma busca da chaves secretas RSA mais específica.

Sabemos que a chave pública está declarada como um objeto ANS.1 RSAPrivateKey (ver Figura 2.1) com os seguintes campos: version, modulus $N$, publicExpoent e, privateExpoent $d$, prime $1 r_{1}$, prime2 $r_{2}$, expoent1 $d \bmod \left(r_{1}-1\right)$, expoent2 $d \bmod \left(r_{2}-1\right)$, coefficient $r_{2}^{-1} \bmod r_{1}$ e alguma outra informação, portanto ele é codificado usando Regras de Codificação Básicas ou BER (Basic Encoding Rules) com a qual nossa chave secreta é expressada em uma cadeia de octetos (bytes) para facilitar a transmissão e interpretação do mesmo.

30

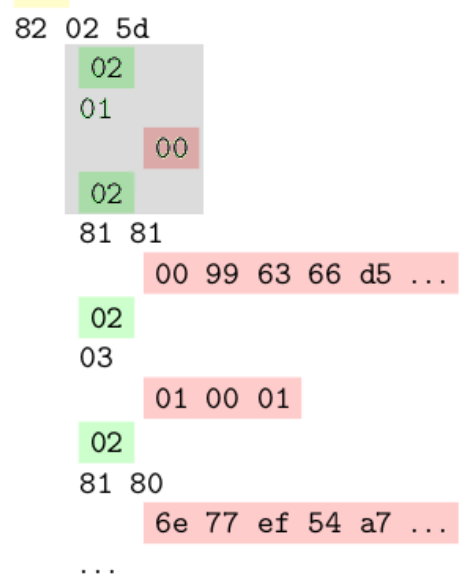

SEQUENCE

length: 605 bytes

INTEGER

length: 1 byte

(version)

INTEGER

length: 129 bytes

(n)

INTEGER

length: 3 bytes

(e)

INTEGER

length: 128 bytes

(d)

Figura 2.4: Codificação BER da chave secreta RSA. A parte sombreada mostra a cadeia 02-01-00-02. (Fonte: Center for Information Technology at Princeton University)

A codificação BER especifica um formato auto-descrição e auto-delimitação para codificar estrutura de dados ANS.1. Cada elemento é codificado com os seguintes campos: tipo de dado, tamanho de dado e o dado mesmo. Este tipo de codificações permite aos destinatários decodificar a informação ANS.1 a partir de uma cadeia de bytes sem ter um pre-conhecimento do tamanho, conteúdo o significado semântico dos dados. Na Figura 2.4 podemos ver a codificação de uma chave secreta RSA.

Para encontrar todas a estruturas de chaves secretas RSA na memória devemos fazer uma busca da sua codificação BER. Um jeito é procurar a cadeia de octetos 02-01-00-02 onde os três primeiros octetos representam a codificação BER da variável version, 0x02 especifica que é um inteiro, 0x01 que seu tamanho é de um byte e 0x00 que o RSA não é multi-primo. E o último octeto 0x02 especifica o tipo de dado da variável seguinte modulus que é um inteiro (veja na Figura 2.4). Já tendo todas as estruturas de chaves secretas RSA agora devemos determinar a chave secreta que estamos procurando, para conseguir isso só pegamos a estrutura que contém o valor $N$.

A busca usando a codificação BER foi explicado para imagens perfeitas da DRAM, mas pode ser adaptado para imagens com bits deteriorados. Primeiro fazemos uma busca das estruturas de chaves RSA procurando cadeias com distância de Hamming ${ }^{9}$ baixa a 02-01-00-02. Conseguido isto procuramos nas estruturas obtidas o valor de variável modulus que tenha uma distância de Hamming baixa com o valor público $N$. Se encontrarmos determinada estrutura podemos recuperar os valores restantes da chave secreta $\tilde{s k}\left\langle N, e, \tilde{p}, \tilde{q}, \tilde{d}, \tilde{d}_{p}, \tilde{d}_{q}, q^{-1}\right\rangle$ com o valor $\delta$ representando a fração de bits corretos do mesmo $\left[\mathrm{HSH}^{+} 09\right]$. Para encontrar uma chave secreta $\tilde{s k}$ do criptossistema RSA Multiprimo podemos aplicar os mesmos passos mas a busca é feita com relação à cadeia 02-00-00-02.

\footnotetext{
${ }^{9}$ Definido como a quantidade de bits diferentes entre dois valores
} 


\section{Capítulo 3}

\section{Algoritmo de Reconstrução da Chave Secreta do Criptossistema RSA}

Como foi explicado no capítulo anterior, podemos obter uma imagem residual da memória DRAM usando uma pequena aplicação ou sistema de boot. Usando essa imagem residual é possível identificar as chaves secretas do criptossistema RSA e poder obter uma chave secreta

$$
\tilde{s k}\left\langle N, e, \tilde{d}, \tilde{r_{1}}, \tilde{r_{2}}, \tilde{d_{1}}, \tilde{d_{2}}, \tilde{r_{2}}{ }^{-1},\left\langle\tilde{r_{3}}, \tilde{d_{3}}, \tilde{t_{3}}\right\rangle, \ldots,\left\langle\tilde{r_{u}}, \tilde{d_{u}}, \tilde{t_{u}}\right\rangle\right\rangle
$$

com uma fração $\delta$ de bits corretos de

$$
s k\left\langle N, e, d, r_{1}, r_{2}, d_{1}, d_{2}, r_{2}{ }^{-1},\left\langle r_{3}, d_{3}, t_{3}\right\rangle, \ldots,\left\langle r_{u}, d_{u}, t_{u}\right\rangle\right\rangle .
$$

Este capítulo está focado à reconstrução da chave secreta $s k$ aproveitando as relações matemáticas existentes entre a chave secreta $s k$ e a chave pública $p k$ que são especificadas no PKCS \#1 e ao conhecimento de uma fração $\delta$ de bits corretos em $\tilde{s k}$, em outras palavras temos conhecimento da posição e o valor de uma porcentagem $\delta$ de bits.

As relações matemáticas entre as chaves $s k$ e $p k$ estão definidas por

$$
\begin{array}{rlrl}
N & =\prod_{i=1}^{u} r_{i} \\
e . d & =1 \quad(\bmod \phi(N)) \\
e . d_{i} & =1 \quad\left(\bmod r_{i}-1\right) \text { para } 1 \leq i \leq u \\
r_{2} \cdot r_{2}^{-1} & =1 \quad\left(\bmod r_{1}\right) \\
\prod_{j=1}^{i-1} r_{j} \cdot t_{i} & =1 \quad & \left(\bmod r_{i}\right) \text { para } 3 \leq i \leq u
\end{array}
$$

as quais expressadas na sua forma matemática não modular temos

$$
\begin{aligned}
N & =\prod_{i=1}^{u} r_{i} \\
e . d & =1+k \prod_{i=1}^{u}\left(r_{i}-1\right) \\
e . d_{i} & =1+k_{i}\left(r_{i}-1\right) \text { para } 1 \leq i \leq u \\
r_{2} \cdot r_{2}{ }^{-1} & =1+g \cdot r_{1} \\
\prod_{j=1}^{i-1} r_{j} . t_{i} & =1+g_{i} \cdot r_{i} \text { para } 3 \leq i \leq u .
\end{aligned}
$$


Observa-se que nas equações acima, que além de ter as variáveis de $p k$ (que são conhecidas) e de $s k$ (onde temos alguns bits corretos deles em $\tilde{s k}$ ), temos $2 u$ novas variáveis: $k, k_{1}, k_{2}, \ldots, k_{u}, g, g_{3}, g_{4}, \ldots, g_{u}$, as quais são inteiros positivos e devem ser pre-calculadas para poder aplicar o algoritmo de reconstrução.

Na Equação (3.2), sabemos que $e, d \in \mathbb{Z}_{\phi(N)}^{*}$ portanto $d<\phi(N)=\prod_{i=1}^{u}\left(r_{i}-1\right)$, sendo assim para manter a igualdade nessa equação o valor de $k$ deve ser menor que $e(k<e)$. Aplicando conceitos similares podemos limitar o valor de $k_{i}$ analisando a Equação (3.3) para seu respectivo valor de $i$. Sabemos que $d_{i}<r_{i}-1$ (já que $d_{i}=d \bmod \left(r_{i}-1\right)$ ) portanto para manter a igualdade nessas equações os valores para $k_{i}$ para $1 \leq i \leq u$ devem ser menores que $e\left(k_{i}<e\right)$. Como sabemos que o valor do expoente de encriptação $e$ é pequeno, isso permite testar todos os possíveis valores para $k$ e $k_{i}$ mas isso seria uma perda de tempo já que nas três primeiras equações RSA existem algumas relações que permitem calcular seus valores eficientemente. Mas isso não acontece para as variáveis $g_{1}, g_{3}, \ldots, g_{u}$.

Sabemos que $r_{2}$ e $r_{1}$ têm o mesmo tamanho (devido a que o módulo $N$ é balanceado), portanto podemos afirmar pela Equação (3.4) que o valor de $g$ tem o mesmo tamanho que o valor de $r_{2}{ }^{-1}$, o qual sempre tem o mesmo tamanho que $r_{2}$, portanto a variável $g$ é quase sempre do mesmo tamanho dos primos $r_{i}$. Na Equação (3.5) é claro que a variável $g_{i}$ é de maior tamanho que os primos $r_{i}$. Sabendo que os valores de $g$ e $g_{i}$ são valores grandes, isso dificulta seu cálculo ou poder testar todos seus possíveis valores. Além disso não foi encontrada nenhuma relação entre as equações RSA que permitam seu cálculo. Portanto as equações (3.4) e (3.5) não são consideradas para o algoritmo de reconstrução, o qual nos deixa só com as seguintes equações RSA

$$
\begin{aligned}
N & =\prod_{i=1}^{u} r_{i} \\
e . d & =1+k \prod_{i=1}^{u}\left(r_{i}-1\right) \\
\text { e.d } & =1+k_{i}\left(r_{i}-1\right) \text { para } 1 \leq i \leq u .
\end{aligned}
$$

Em outras palavras, a porcentagem de bits que conhecemos nas variáveis $\left\langle\tilde{r_{2}}{ }^{-1}, \tilde{t_{3}}, \tilde{t_{4}}, \ldots, \tilde{t_{u}}\right\rangle$ não são aproveitados, portanto o algoritmo de reconstrução só aproveita a porcentagem de bits corretos em $\tilde{s k}\left\langle\tilde{d}, \tilde{r_{1}}, \tilde{r_{2}}, \tilde{d_{1}}, \tilde{d_{2}},\left\langle\tilde{r_{3}}, \tilde{d}_{3}\right\rangle, \ldots,\left\langle\tilde{r_{u}}, \tilde{d_{u}}\right\rangle\right\rangle$.

O capítulo presente está dividido nas seguintes seções. A primeira Seção 3.1 vamos nos focar no cálculo das variáveis $k$ e $k_{i}$. Na segunda Seção 3.2 vamos fazer algumas correções nos bits de algumas variáveis de $\tilde{s k}$. Na terceira Seção 3.3 vamos analisar a dependência dos bits da chave secreta $s k$ usando o Lema de Hensel. E por último na quarta Seção 3.4 explicaremos do algoritmo de reconstrução da chave secreta contendo uma análise do seu comportamento e sua complexidade.

\subsection{Cálculo de Variáveis Auxiliares}

Vale lembrar que a análise é feita para um e pequeno cujo valor é primo. Para poder calcular os valores de $k$ e $k_{i}$, o primeiro passo a fazer é aplicar módulo $e(\bmod e)$ às equações $(3.1),(3.2) \mathrm{e}$ (3.3) onde obtemos que:

$$
\begin{aligned}
N & \equiv \prod_{i=1}^{u} r_{i} \\
0 & \equiv 1+k \prod_{i=1}^{u}\left(r_{i}-1\right) \\
0 & \equiv 1+k_{i}\left(r_{i}-1\right) \text { para } 1 \leq i \leq u .
\end{aligned}
$$


Observa-se que temos equações no anel comutativo $Z_{e}^{*}$ onde podemos determinar duas novas equações que vão permitir o cálculo de $k$ e $k_{i}$, onde elas são obtidas substituindo o valor de $r_{i}-1 \equiv-\left(k_{i}\right)^{-1}$ da Equação (3.8) para $1 \leq i \leq u$ na Equação (3.7)

$$
\begin{array}{rlr}
0 & \equiv 1+k \prod_{i=1}^{u}\left[-1\left(k_{i}\right)^{-1}\right] & \\
0 & \equiv 1+(-1)^{u} k \prod_{i=1}^{u}\left(k_{i}\right)^{-1} & \text { Constante }-1 \text { em um produtório } \\
0 & \equiv \prod_{i=1}^{u} k_{i}+(-1)^{u} k & \text { Multiplicando } \prod_{i=1}^{u} k_{i} \\
-(-1)^{u} k & \equiv \prod_{i=1}^{u} k_{i} &
\end{array}
$$

e o valor de $r_{i} \equiv 1-\left(k_{1}\right)^{-1}$ da Equação (3.8) para $1 \leq i \leq u$ na Equação (3.6).

$$
N \equiv \prod_{i=1}^{u}\left[1-\left(k_{i}\right)^{-1}\right]
$$

Analisando as equações (3.9) e (3.10) no anel comutativo $\mathbb{Z}_{e}^{*}$ podemos calcular os valores de $k$ e $k_{i}$. A análise será feito nas subseções seguintes.

\subsubsection{Caso 1}

No artigo [Per05], Percival estudou o número de soluções que temos para os valores de $\left\langle k, k_{1}, k_{2}\right\rangle$ do criptossistema RSA básico determinando um total de $e$ soluções para $\left\langle k, k_{1}, k_{2}\right\rangle$. Para nosso estudo sobre o criptossistema RSA multi-primo vamos definir como caso 1 ao estudo onde é determinado o total de soluções para $\left\langle k, k_{1}, k_{2}, \ldots, k_{u}\right\rangle$. Para este estudo vamos analisar a Equação (3.10) para determinar os valores de $k_{1}, k_{2}, . ., k_{u}$ e com esses valores podemos calcular o valor de $k$ usando a Equação (3.9).

Percival para calcular o número de soluções de $k_{1}, k_{2}$ na Equação (3.10) com $u=2$

$$
N \equiv\left[1-\left(k_{1}\right)^{-1}\right]\left[1-\left(k_{2}\right)^{-1}\right]
$$

simplesmente atribuiu todos os possíveis valores para $k_{1}$ dando origem a e equações (já que $0 \leq k_{i} \leq$ e) onde para cada $k_{1}$ é calculado seu valor correspondente para $k_{2}$ na Equação (3.10), e com $k_{1}, k_{2}$ é possível calcular o valor de $k$ na Equação (3.9). Percival afirmou que temos um total de $e$ possíveis soluções para $\left\langle k, k_{1}, k_{2}\right\rangle$ devidos ao número de equações geradas no momento de atribuir todos os possíveis valores para $k_{1}$, mas esse número de soluções é um valor aproximado como mostraremos a seguir.

Com relação ao criptossistema RSA multi-primo temos na Equação (3.10) que

$$
N \equiv \prod_{i=1}^{u}\left[1-\left(k_{i}\right)^{-1}\right],
$$

onde sabemos que o valor de $\mathrm{N}$ é a multiplicação de $u$ primos de tamanho maior que $e$ portanto 
podemos afirmar que $N \not \equiv 0 \bmod e$ já que $e \nmid N$, então temos que

$$
\begin{array}{rr}
\prod_{i=1}^{u}\left[1-\left(k_{i}\right)^{-1}\right] \not \equiv 0 & \text { O produtório não é equivalente a } 0 \text { já que } e \nmid N \\
1-\left(k_{i}\right)^{-1} \not \equiv 0 & \text { O fatores do produtório não são equivalentes a } 0 \\
k_{i}-1 \not \equiv 0 & \text { Multiplicando por } k_{i} \\
k_{i} \not \equiv 1 & \text { Somando a unidade } 1 .
\end{array}
$$

Observar-se que para $k_{i}$ não pode-se atribuir o valor de 1 . Além disso no processo estamos calculando o inverso multiplicativo de $k_{i}$ portanto $k_{i} \not \equiv 0$ (veja propriedades de anel comutativo no Apêndice A.1.5), portanto podemos afirmar que $k_{i}$ só pode ter $e-2$ possíveis valores $\left(2 \leq k_{i} \leq e-1\right.$ para $1 \leq i \leq u)$.

Vamos definir a função $\alpha(u)$ que retorna o número de soluções para $k_{1}, k_{2}, \ldots, k_{u}$ onde para calcular seus valores simplesmente atribuímos valores para as variáveis $k_{1}, k_{2}, \ldots, k_{u-1}$ onde cada variável $k_{i}$ tem $e-2$ valores possíveis, portanto temos um total de $(e-2)^{u-1}$ equações onde para cada uma delas devemos calcular o valor para seu correspondente $k_{u}$.

$$
\begin{array}{rlr}
N & \equiv \prod_{i=1}^{u}\left[1-\left(k_{i}\right)^{-1}\right] & \\
N & \equiv\left[1-\left(k_{u}\right)^{-1}\right] \prod_{i=1}^{u-1}\left[1-\left(k_{i}\right)^{-1}\right] & \text { Propriedade de } \prod \\
N \prod_{i=1}^{u-1}\left[1-\left(k_{i}\right)^{-1}\right]^{-1} & \equiv 1-\left(k_{u}\right)^{-1} & \text { Multiplicando } \prod_{i=1}^{u-1}\left[1-\left(k_{i}\right)^{-1}\right]^{-1} \\
N \prod_{i=1}^{u-1}\left[1-\left(k_{i}\right)^{-1}\right]^{-1}-1 & \equiv-\left(k_{u}\right)^{-1} & \text { Somando -1 } \\
1-N \prod_{i=1}^{u-1}\left[1-\left(k_{i}\right)^{-1}\right]^{-1} & \equiv\left(k_{u}\right)^{-1} & \text { Multiplicando -1 }
\end{array}
$$

Portanto temos solução para $k_{u}$ só se o valor $k_{u}^{-1}$ não for equivalente a 0 . Em outras palavras, do total de $(e-2)^{u-1}$ equações temos algumas equações que não vão ter solução para $k_{u}$ só se o valor de $1-N \prod_{i=1}^{u-1}\left[1-\left(k_{i}\right)^{-1}\right]^{-1}$ for equivalente a 0 .

$$
\begin{array}{rlr}
1-N \prod_{i=1}^{u-1}\left[1-\left(k_{i}\right)^{-1}\right]^{-1} & \equiv 0 & \text { Definição de }\left(k_{u}\right)^{-1} \\
N \prod_{i=1}^{u-1}\left[1-\left(k_{i}\right)^{-1}\right]^{-1} & \equiv 1 & \text { Multiplicando } \prod_{i=1}^{u-1}\left[1-\left(k_{i}\right)^{-1}\right] \\
N & \equiv \prod_{i=1}^{u-1}\left[1-\left(k_{i}\right)^{-1}\right]
\end{array}
$$

Por conseguinte, temos atribuímos valores a $k_{1}, k_{2}, \ldots, k_{u-1}$ resultando $(e-2)^{u-1}$ equações das quais não vão ter soluções para $k_{u}$ se $N \equiv \prod_{i=1}^{u-1}\left[1-\left(k_{i}\right)^{-1}\right]$, o qual o número de vezes que acontece isso está dado por $\alpha(u-1)$, portanto o número de soluções possíveis para $k_{1}, k_{2}, \ldots, k_{u}$ está definido como

$$
\alpha(u)=(e-2)^{u-1}-\alpha(u-1) .
$$

No caso base $(u=1)$ temos que $N \equiv\left[1-\left(k_{1}\right)^{-1}\right]$ e para que exista solução para $k_{1}$ deve cumprir 
que $N \not \equiv 1 \bmod e$. Com o caso $u=1$ já analisado podemos definir completamente a função $\alpha(u)$.

$$
\alpha(u)=\left\{\begin{array}{lll}
0 & \text { se } u=1 \text { e } N \equiv 1 & \bmod e \\
1 & \text { se } u=1 \text { e } N \not \equiv 1 & \bmod e \\
(e-2)^{u-1}-\alpha(u-1) & \text { se } u>1
\end{array}\right\}
$$

Sabemos que temos um total de $\alpha(u)$ soluções possíveis para $k_{1}, k_{2}, \ldots k_{u}$ onde para cada uma delas existe um valor $k$ que pode ser determinado na Equação (3.9). O valor de $k$ sempre existe para cada solução possível de $k_{1}, k_{2}, \ldots k_{u}$ já que $\mathbb{Z}_{e}^{*}$ é fechado (Veja o Teorema 1), portanto o número de soluções possíveis para $\left\langle k, k_{1}, k_{2}, \ldots k_{u}\right\rangle$ está denotado pela função $\alpha(u)$. A seguir daremos alguns exemplos mostrando a corretude da função $\alpha(u)$.

\section{Exemplos}

1. Para o criptossistema RSA básico $(u=2)$

- Gerando uma criptossistema RSA

$r_{1}=11, \quad r_{2}=5, \quad N=55, \quad \phi(N)=40, \quad e=7, \quad d=23$

- Calculando os expoentes de decriptação $d_{i} \mathrm{~s}$

$$
d_{1}=23 \bmod 10=3 \quad d_{2}=23 \bmod 4=3
$$

- Calculando os valores corretos de $\left\langle k_{1}, k_{2}\right\rangle$

$$
\begin{array}{cc}
e d_{1}=1+k_{1}\left(r_{1}-1\right) & e d_{2}=1+k_{2}\left(r_{2}-1\right) \\
7 * 3=1+k_{1}(10) & 7 * 3=1+k_{2}(4) \\
k_{1}=2 & k_{2}=5
\end{array}
$$

portanto $\left\langle k_{1}, k_{2}\right\rangle=\langle 2,5\rangle$

- Calculando o total de soluções possíveis soluções para $\left\langle k_{1}, k_{2}\right\rangle$

\begin{tabular}{|c||cc|}
\hline$\#$ sol & $k_{1}$ & $k_{2}$ \\
\hline \hline $1^{\circ}$ & 2 & 5 \\
\hline \hline $2^{\circ}$ & 3 & 6 \\
- & 4 & $\nexists$ \\
$3^{\circ}$ & 5 & 2 \\
$4^{\circ}$ & 6 & 3 \\
\hline
\end{tabular}

Temos 4 soluções para $\left\langle k_{1}, k_{2}\right\rangle$ onde a $1^{\text {a }}$ é a correta.

- Calculando o total de soluções possíveis para $\left\langle k_{1}, k_{2}\right\rangle$ utilizando a definição da função $\alpha(u)$.

Calculando $\alpha(u)$ onde $u=2$ onde

$$
\begin{gathered}
\alpha(u)=(e-2)^{u-1}-\alpha(u-1) \\
\alpha(2)=(5)^{1}-\alpha(1)
\end{gathered}
$$

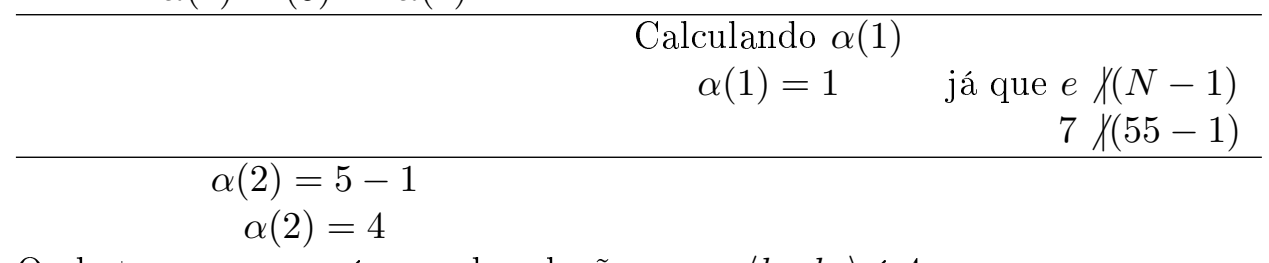

Onde temos que o número de soluções para $\left\langle k_{1}, k_{2}\right\rangle$ é 4 .

2. Para o criptossistema RSA multi-primo $(u=3)$

- Gerando um criptossistema RSA 3-primos

$r_{1}=11, \quad r_{2}=5, \quad r_{3}=3, \quad N=165, \quad \phi(N)=80, \quad e=7, \quad d=23$

- Calculando os expoentes de decriptação $d_{i} \mathrm{~s}$

$$
d_{1}=23 \bmod 10=3 \quad d_{2}=23 \bmod 4=3 \quad d_{3}=23 \bmod 2=1
$$


- Calculando os valores corretos de $\left\langle k_{1}, k_{2}\right\rangle$

$$
\begin{array}{ccc}
e d_{1}=1+k_{1}\left(r_{1}-1\right) & e d_{2}=1+k_{2}\left(r_{2}-1\right) & e d_{3}=1+k_{3}\left(r_{3}-1\right) \\
7 * 3=1+k_{1}(10) & 7 * 3=1+k_{2}(4) & 7 * 1=1+k_{3}(2) \\
k_{1}=2 & k_{2}=5 & k_{3}=3
\end{array}
$$

portanto $\left\langle k_{1}, k_{2}, k_{3}\right\rangle=\langle 2,5,3\rangle$

- Calculando o total de soluções possíveis para $\left\langle k_{1}, k_{2}, k_{3}\right\rangle$

\begin{tabular}{|c||ccc|}
\hline$\#$ sol & $k_{1}$ & $k_{2}$ & $k_{3}$ \\
\hline \hline $1^{\circ}$ & 2 & 2 & 6 \\
$2^{\circ}$ & 2 & 3 & 5 \\
$3^{\circ}$ & 2 & 4 & 4 \\
\hline \hline $4^{\circ}$ & 2 & 5 & 3 \\
\hline \hline $5^{\circ}$ & 2 & 6 & 2 \\
$6^{\circ}$ & 3 & 2 & 5 \\
$7^{\circ}$ & 3 & 3 & 6 \\
- & 3 & 4 & $\nexists$ \\
$8^{\circ}$ & 3 & 5 & 2 \\
\hline
\end{tabular}

\begin{tabular}{|c||ccc|}
\hline$\#$ sol & $k_{1}$ & $k_{2}$ & $k_{3}$ \\
\hline \hline $9^{\circ}$ & 3 & 6 & 3 \\
$10^{\circ}$ & 4 & 2 & 4 \\
- & 4 & 3 & $\nexists$ \\
$11^{\circ}$ & 4 & 4 & 2 \\
$12^{\circ}$ & 4 & 5 & 6 \\
$13^{\circ}$ & 4 & 6 & 5 \\
$14^{\circ}$ & 5 & 2 & 3 \\
$15^{\circ}$ & 5 & 3 & 2 \\
$16^{\circ}$ & 5 & 4 & 6 \\
\hline
\end{tabular}

\begin{tabular}{|c||ccc|}
\hline \# sol & $k_{1}$ & $k_{2}$ & $k_{3}$ \\
\hline \hline- & 5 & 5 & $\nexists$ \\
$17^{\circ}$ & 5 & 6 & 4 \\
$18^{\circ}$ & 6 & 2 & 2 \\
$19^{\circ}$ & 6 & 3 & 3 \\
$20^{\circ}$ & 6 & 4 & 5 \\
$21^{\circ}$ & 6 & 5 & 4 \\
- & 6 & 6 & $\nexists$ \\
& & & \\
& & & \\
\hline
\end{tabular}

Temos 21 soluções para $\left\langle k_{1}, k_{2}, k_{3}\right\rangle$ onde a $4^{\mathrm{a}}$ é a correta.

- Calculando o total de soluções possíveis para $\left\langle k_{1}, k_{2}, k_{3}\right\rangle$ utilizando a definição da função $\alpha(u)$.

Calculando $\alpha(u)$ onde $u=3$ onde

$$
\begin{array}{cc}
\alpha(u)=(e-2)^{u-1}-\alpha(u-1) & \\
\alpha(3)=(5)^{2}-\alpha(2) & \text { Calculando } \alpha(2) \\
\alpha(u)=(e-2)^{u-1}-\alpha(u-1) \\
\alpha(2)=(5)^{1}-\alpha(1)
\end{array}
$$

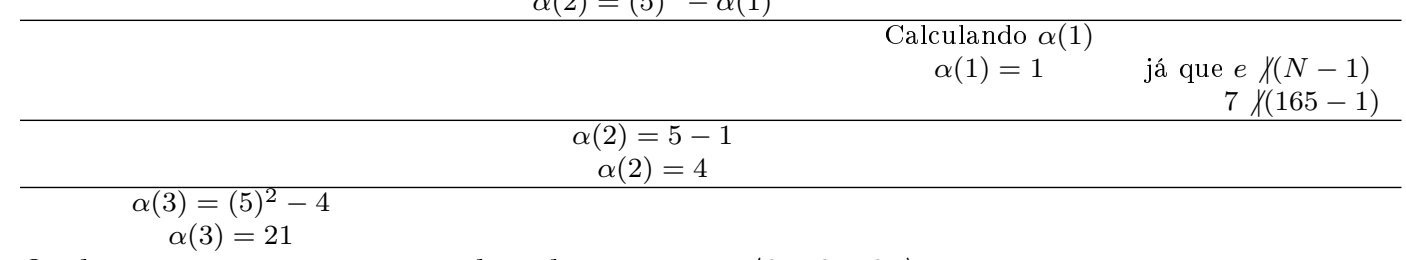

Onde temos que o número de soluções para $\left\langle k_{1}, k_{2}, k_{3}\right\rangle$ é 21 .

\subsubsection{Caso 2}

Com relação ao criptossistema RSA básico, Heninger explica como é possível o cálculo das variáveis $k_{1}, k_{2}$ quando temos que o valor de $k$ é conhecido [HS09]. Para poder estudar esse caso vamos analisar as equações (3.10) e (3.9), mas antes vamos explicar como é possível o cálculo de $k$ [BDF98].

\section{Calculando o Valor de $k$}

Fato 1. Para um e pequeno, a metade dos MSB de d pode ser estimados eficientemente [MSSG10]. Sabemos que o valor $k$ está entre 0 e e, portanto temos $e-1$ possíveis candidatos para a variável $k$, seja $k^{\prime}$ um candidato de $k$ então calculamos:

$$
d_{k^{\prime}}=\frac{k^{\prime}(N+1)+1}{e}
$$

Com a escolha correta de $k^{\prime}=k$ podemos obter os MSB d já que:

$$
\left|d_{k^{\prime}}-d\right|=\frac{k(p+q)}{e}<p+q
$$

O fato acima só é aplicável quando $N$ é a multiplicação de 2 primos. Aplicando os mesmos conceitos podemos estabelecer um novo fato para quando o $N$ for a multiplicação de 2 ou mais primos. 
Fato 2. Para um e pequeno, os $\frac{n}{u} M S B$ de d pode ser estimados eficientemente.

Temos a Equação RSA (3.3) que pode ser expressada do seguinte jeito

$$
\begin{array}{lr}
e d=1+k \prod_{i=1}^{u}\left(r_{i}-1\right)+k N-k \prod_{i=1}^{u} r_{i} & \text { Visto que } k N=k \prod_{i=1}^{u} r_{i} \\
e d=1+k N-k\left(\prod_{i=1}^{u} r_{i}-\prod_{i=1}^{u}\left(r_{i}-1\right)\right) & \text { Fatorando } k \\
d=\frac{1+k N}{e}-\frac{k}{e}\left(\prod_{i=1}^{u} r_{i}-\prod_{i=1}^{u}\left(r_{i}-1\right)\right) & \text { Dividindo } e
\end{array}
$$

Onde vamos definir $d=d_{0}+d_{1} \operatorname{com} d_{0}=\frac{1+k N}{e}$ e $d_{1}=-\frac{k}{e}\left(\prod_{i=1}^{u} r_{i}-\prod_{i=1}^{u}\left(r_{i}-1\right)\right)$ portanto podemos afirmar que

$$
\begin{aligned}
\left|d-d_{0}\right|=\left|d_{1}\right| & =\frac{k}{e}\left(\prod_{i=1}^{u} r_{i}-\prod_{i=1}^{u}\left(r_{i}-1\right)\right) \\
\left|d_{1}\right| & =\frac{k}{e}\left(\sum_{i=1}^{u} \frac{N}{r_{i}}-\sum_{1 \leq i}^{u-1} \sum_{i \leq j}^{u} \frac{N}{r_{i} r_{j}}+\ldots-(-1)^{u}\right) \\
\left|d_{1}\right| & \leq \frac{k}{e}\left(\sum_{i=1}^{u} \frac{N}{r_{i}}\right) \\
\left|d_{1}\right| & \leq \sum_{i=1}^{u} \frac{N}{r_{i}} \\
\left|d_{1}\right| & \leq \sum_{i=1}^{u} \frac{N}{r_{\min }} \\
\left|d_{1}\right| & \leq u \frac{N}{r_{\min }}
\end{aligned}
$$

Com o resultado acima podemos afirmar que o número de máximo bits que pode ter $d_{1}\left(l_{d_{1}}\right)$ está dado por

$$
\begin{aligned}
& l_{d_{1}} \approx \lg \left(u \frac{N}{r_{\text {min }}}\right) \\
& l_{d_{1}} \approx \lg (u)+\lg (N)-\lg \left(r_{\text {min }}\right) \\
& l_{d_{1}} \approx \lg (u)+n-\frac{n}{u} .
\end{aligned}
$$

Sabemos que número de bits de $d_{0}\left(k^{\prime}\right)=\frac{1+k^{\prime} N}{e} \leq N$ é dado por $n$ bits, portanto podemos afirmar que $d_{0}\left(k^{\prime}\right)$ para um $k^{\prime}=k$ tem os bits a partir da posição $\lg (u)+n-\frac{n}{u}+1$ do expoente de decifração $d$, isso acontece porque sua diferença $\left(\left|d-d_{0}\right|=\left|d_{1}\right|\right)$ tem um tamanho máximo de $\lg (u)+n-\frac{n}{u}$ bits. Com o Fato 2 já demostrado, procedemos a calcular o valor de $k$.

Temos uma fração $\delta$ de bits conhecidos em $\tilde{s k}\left\langle\tilde{d}, \tilde{r_{1}}, \tilde{r_{2}}, \tilde{d}_{1}, \tilde{d}_{2},\left\langle\tilde{r_{3}}, \tilde{d_{3}}\right\rangle, \ldots,\left\langle\tilde{r_{u}}, \tilde{d_{u}}\right\rangle\right\rangle$ portanto podemos asseverar que temos um total de $\delta n$ bits corretos no expoente de decifração $\tilde{d}$, os quais são aproveitados para calcular o valor de $k$.

Primeiro calculamos

$$
d_{0}\left(k^{\prime}\right)=\frac{1+k^{\prime} N}{e} \text { para } 1 \leq k^{\prime} \leq e-1,
$$


onde para cada valor $d_{0}\left(k^{\prime}\right)$ podemos determinar a distância de Hamming entre os bits conhecidos de $\tilde{d}$ e os bits $\frac{n}{u}-l g(u)$ MSB de $d_{0}\left(k^{\prime}\right)$.

$$
H\left(k^{\prime}\right)=\sum_{j=n-\frac{n}{u}}^{n} d_{0}\left(k^{\prime}\right)[j] \oplus \tilde{d}[j] \text { só se o bit } \tilde{d}[j] \text { é conhecido }
$$

Os valores dos $k^{\prime}$ s onde seus $H(k)^{\prime}$ s tenham a menor valor serão as melhores escolhas para $k$.

$$
k=\left\{k^{\prime} \mid H\left(k^{\prime}\right)=\min (H(1), H(2), . ., H(e-2), H(e-1))\right\}
$$

Vale frisar que enquanto o valor de $u$ seja maior temos menos bits para poder determinar o valor de $k$.

\section{Calculando os Valores de $k_{i}$}

O procedimento para calcular os valores de $k_{1}, \ldots, k_{u}$ tendo o valor de $k$, é atribuir valores para os variáveis $k_{1}, \ldots, k_{u-2}$ nas equações (3.10) e (3.9) portanto vamos gerar $(e-2)^{u-2}$ sistemas de duas equações com duas variáveis a achar, $k_{u-1}$ e $k_{u}$. Nesse sistema de equações podemos substituir a variável $k_{u}$ definida segundo á Equação (3.9) em (3.10) e assim ter uma soa equação e a variável $k_{u-1}$ a calcular. Se existir alguma solução para $k_{u-1}$ procedemos a calcular o valor de $k_{u}$ em qualquer das equações (3.10) e (3.9).

Temos as equações (3.10) e (3.9)

$$
\begin{array}{ll}
N \equiv \prod_{i=1}^{u}\left[1-\left(k_{i}\right)^{-1}\right] & -(-1)^{u} k \equiv \prod_{i=1}^{u} k_{i} \\
N \equiv\left[1-\left(k_{u-1}\right)^{-1}\right]\left[1-\left(k_{u}\right)^{-1}\right] \prod_{i=1}^{u-2}\left[1-\left(k_{i}\right)^{-1}\right] & -(-1)^{u} k \equiv k_{u-1} k_{u} \prod_{i=1}^{u-2} k_{i} .
\end{array}
$$

onde vamos atribuir todos os possíveis valores para $k_{1}, k_{2}, \ldots, k_{u-2}$

$$
N \equiv\left[1-\left(k_{u-1}\right)^{-1}\right]\left[1-\left(k_{u}\right)^{-1}\right] L \quad-(-1)^{u} k \equiv k_{u-1} k_{u} M
$$

onde $L \equiv \prod_{i=1}^{u-2}\left[1-\left(k_{i}\right)^{-1}\right]$ e $M \equiv \prod_{i=1}^{u-2} k_{i}$. De acordo com a segunda equação podemos determinar que $k_{u}$ é dado por

$$
\begin{aligned}
-(-1)^{u} k & \equiv k_{u-1} k_{u} M \\
(-1)^{u+1} k k_{u-1}^{-1} M^{-1} & \equiv k_{u}
\end{aligned}
$$

para ser substituída em $N \equiv\left[1-\left(k_{u-1}\right)^{-1}\right]\left[1-\left(k_{u}\right)^{-1}\right] L$.

$$
\begin{aligned}
N & \equiv\left[1-\left(k_{u-1}\right)^{-1}\right]\left[1-\left((-1)^{u+1} k k_{u-1}^{-1} M^{-1}\right)^{-1}\right] L & \\
N(-1)^{u+1} k k_{u-1}^{-1} M^{-1} & \equiv\left[1-\left(k_{u-1}\right)^{-1}\right]\left[(-1)^{u+1} k k_{u-1}^{-1} M^{-1}-1\right] L & \text { Multiplicando }(-1)^{u+1} k k_{u-1}^{-1} M^{-1} \\
N(-1)^{u} k k_{u-1} M^{-1} L^{-1} & \equiv\left[k_{u-1}-1\right]\left[k_{u-1}+(-1)^{u} k M^{-1}\right] & \text { Multiplicando }(-1) k_{u-1}^{2} L^{-1} \\
N(-1)^{u} k k_{u-1} M^{-1} L^{-1} & \equiv k_{u-1}^{2}+\left[(-1)^{u} k M^{-1}-1\right] k_{u-1}-(-1)^{u} k M^{-1} &
\end{aligned}
$$

Da equação acima obtemos a seguinte equação quadrática em $\mathbb{Z}_{e}^{*}$

$$
0 \equiv k_{u-1}^{2}-\left[(-1)^{u} k M^{-1}\left[N L^{-1}-1\right]+1\right] k_{u-1}-(-1)^{u} k M^{-1} .
$$

Para o cálculo das soluções de $k_{u-1}$ pode-se ler a Seção A.2.2. Como temos uma equação quadrática então temos no máximo duas raízes para $k_{u-1}$ (veja a Definição 13), portanto procedemos a calcular o valor de $k_{u}$ para cada valor de $k_{u-1}$ em qualquer das Equações (3.10) ou (3.9).

Vamos definir $\beta(u)$ como o número de soluções para $\left\langle k_{1}, k_{2}, \ldots, k_{u}\right\rangle$ onde o valor de $k$ é conhecido. Lembramos então que temos $(e-2)^{u-2}$ equações que são quadráticas mas algumas dessas equações 
vão ter solução ou não dependendo do valor $Y$ (ver o Apêndice A.2.2) definido por

$$
Y \equiv\left(-k(-1)^{u}\left(N L^{-1}-M\right)+1\right)^{2}+4\left(k(-1)^{u} M\right) .
$$

Se $Y$ for resíduo quadrado temos solução caso contrário não. Como temos $(e-2)^{u-2}$ equações quadráticas não foi possível encontrar um padrão para contabilizar quantas delas têm solução e quantas não, portanto não foi determinado o número exato de soluções para $\left\langle k_{1}, k_{2}, \ldots, k_{u}\right\rangle$. Mas podemos calcular um limite superior para $\beta(u)$ onde podemos dizer que as $(e-2)^{u-2}$ equações quadráticas sempre vão ter solução, portanto cada uma das equações vai ter duas raízes para $k_{u-1}$ e para cada valor de $k_{u-1}$ procedemos ao cálculo de seu respectivo $k_{u}$ portanto podemos asseverar que $\beta(u)$ é menor ou igual a $2(e-2)^{u-2}$.

$$
\beta(u) \leq 2(e-2)^{u-2}
$$

Para o Criptossistema RSA básico simplesmente substituímos o valor de $u=2$ nas equações (3.11) e (3.9) onde temos

$$
\begin{aligned}
0 & \equiv k_{1}^{2}-[k[N-1]+1] k_{1}-k \\
-k & \equiv k_{1} k_{2}
\end{aligned}
$$

o qual é o sistema de equações para calcular os valores de $k_{1}$ e $k_{2}$ tendo o valor $k$ [HS09].

A seguir mostraremos alguns exemplos:

\section{Exemplos}

1. Para o criptossistema RSA básico $(u=2)$

- Gerando um criptossistema RSA $r_{1}=11, \quad r_{2}=5, \quad N=55, \quad \phi(N)=40, \quad e=7, \quad d=23$

- Calculando os expoentes de decriptação $d_{i} \mathrm{~s}$

$$
d_{1}=23 \bmod 10=3 \quad d_{2}=23 \bmod 4=3
$$

- Calculando os valores corretos de $\left\langle k_{1}, k_{2}\right\rangle$

$$
\begin{array}{ccc}
e d=1+k\left(r_{1}-1\right)\left(r_{2}-1\right) & e d_{1}=1+k_{1}\left(r_{1}-1\right) & e d_{2}=1+k_{2}\left(r_{2}-1\right) \\
7 * 23=1+k_{1}(10)(4) & 7 * 3=1+k_{1}(10) & 7 * 3=1+k_{2}(4) \\
k=4 & k_{1}=2 & k_{2}=5
\end{array}
$$

portanto $\left\langle k, k_{1}, k_{2}\right\rangle=\langle 4,2,5\rangle$

- Calculando o total de soluções possíveis soluções para $\left\langle k_{1}, k_{2}\right\rangle$ utilizando a Equação (3.11)

\begin{tabular}{|c||ccc|}
\hline$\#$ sol & $k$ & $k_{1}$ & $k_{2}$ \\
\hline \hline $1^{\circ}$ & 4 & 2 & 5 \\
\hline \hline $2^{\circ}$ & 4 & 5 & 2 \\
\hline
\end{tabular}

Temos 2 soluções para $\left\langle k, k_{1}, k_{2}\right\rangle$ onde a $1^{\circ}$ é a correta.

- Calculando o total de soluções possíveis para $\left\langle k, k_{1}, k_{2}\right\rangle$ utilizando a Inequação (3.12).

Calculando $\beta(u)$ onde $u=2$ onde

$$
\begin{gathered}
\beta(2) \leq 2(e-2)^{u-2} \\
\beta(2) \leq 2(7-2)^{2-2} \\
\beta(2) \leq 2
\end{gathered}
$$

Onde temos que o número de soluções para $\left\langle k, k_{1}, k_{2}\right\rangle$ é menor ou igual a 2 .

2. Para o criptossistema RSA multi-primo $(u=3)$

- Gerando um criptossistema RSA 3-primos

$r_{1}=11, \quad r_{2}=5, \quad r_{3}=3, \quad N=165, \quad \phi(N)=80, \quad e=7, \quad d=23$

- Calculando os expoentes de decriptação $d_{i} \mathrm{~s}$

$$
d_{1}=23 \bmod 10=3 \quad d_{2}=23 \bmod 4=3 \quad d_{3}=23 \bmod 2=1
$$


- Calculando os valores corretos de $\left\langle k, k_{1}, k_{2}, k_{3}\right\rangle$

$$
\begin{array}{cccc}
e d=1+k \prod_{i=1}^{3}\left(r_{1}-1\right) & e d_{1}=1+k_{1}\left(r_{1}-1\right) & e d_{2}=1+k_{2}\left(r_{2}-1\right) & e d_{3}=1+k_{3}\left(r_{3}-1\right) \\
7 * 23=1+k_{1}(80) & 7 * 3=1+k_{1}(10) & 7 * 3=1+k_{2}(4) & 7 * 1=1+k_{3}(2) \\
k=2 & k_{1}=2 & k_{2}=5 & k_{3}=3
\end{array}
$$

portanto $\left\langle k_{1}, k_{2}, k_{3}\right\rangle=\langle 2,5,3\rangle$

- Calculando o total de soluções possíveis para $\left\langle k_{1}, k_{2}, k_{3}\right\rangle$ utilizando a Equação (3.10)

\begin{tabular}{|c||cccc|}
\hline$\#$ sol & $k$ & $k_{1}$ & $k_{2}$ & $k_{3}$ \\
\hline \hline $1^{\circ}$ & 2 & 2 & 3 & 5 \\
\hline \hline $2^{\circ}$ & & & 5 & 3 \\
$3^{\circ}$ & 2 & 3 & 5 & 2 \\
$4^{\circ}$ & & & 2 & 5 \\
& 2 & 4 & $\nexists$ & \\
$5^{\circ}$ & 2 & 5 & 2 & 3 \\
$6^{\circ}$ & & & 3 & 2 \\
& 2 & 6 & $\nexists$ & \\
\hline
\end{tabular}

Temos 6 soluções para $\left\langle k_{1}, k_{2}, k_{3}\right\rangle$ onde a primeira é a correta.

- Calculando o total de soluções possíveis para $\left\langle k, k_{1}, k_{2}, k_{3}\right\rangle$ utilizando a Inequação (3.12).

Calculando $\beta(u)$ onde $u=3$ onde

$$
\begin{gathered}
\beta(3) \leq 2(e-2)^{u-2} \\
\beta(3) \leq 2(7-2)^{3-2} \\
\beta(2) \leq 10
\end{gathered}
$$

Onde temos que o número de soluções para $\left\langle k, k_{1}, k_{2}, k_{3}\right\rangle$ é menor ou igual a 10.

\subsection{Correção de Alguns Bits}

Já calculado as variáveis auxiliares $k, k_{1}, k_{2}, \ldots, k_{u}$ então procedemos a corrigir alguns bits na chave secreta $\tilde{s k}\left\langle\tilde{d}, \tilde{r_{1}}, \tilde{r_{2}}, \tilde{d_{1}}, \tilde{d_{2}}\left\langle\tilde{r_{3}}, \tilde{d_{3}}\right\rangle \ldots\left\langle\tilde{r_{u}}, \tilde{d_{u}}\right\rangle\right\rangle$. As correções explicadas a seguir são feitas com conhecimentos matemáticos básicos.

Sabemos que $r_{i}$ são números primos portanto podemos corrigir

$$
\tilde{r_{i}}[0]=1 \text { para } 1 \leq i \leq u \text {. }
$$

Outra correção a ser feita é sobre os $\frac{n}{u}-l g(u)$ MSB de $\tilde{d}$ já que temos o valor de $k$ e a partir dele é possível calcular os $\frac{n}{u}-l g(u)$ MSB de $d$ (foi visto na Seção 3.1.2). Esse processo é opcional já que no processo de execução do algoritmo de reconstrução vamos usar os $\frac{n}{u}$ LSB corretos de $\tilde{d}$. Mas com o conhecimento do valor $k$ podemos corrigir alguns LSB de $\tilde{d}$. Para isso, definimos a função $\tau(x)$ que retorna o máximo expoente de 2 tal que $x$ é dividido por $2^{\tau(x)}$.

$$
\tau(x)=\left\{\text { máximo expoente de } 2 \text { tal que } 2^{\tau(x)} \mid x\right\}
$$

Sabemos que $2 \mid r_{i}-1$ já que $r_{i}$ é primo portanto podemos afirmar

$$
\begin{array}{rr}
2^{u} \mid \prod_{i=1}^{u}\left(r_{i}-1\right) & \text { Multiplicação de } u \text { números pares } \\
2^{u+\tau(k)} \mid k \prod_{i=1}^{u}\left(r_{i}-1\right) & \text { Visto que } 2^{\tau(k)} \mid k \\
2^{u+\tau(k)} \mid e d-1 & \text { Pela Equação (3.2) }
\end{array}
$$


onde $2^{u+\tau(k)} \mid e d-1$ pode ser expressada como

$$
\begin{aligned}
e d-1 & \equiv 0 \quad \bmod 2^{u+\tau(k)} \\
e d & \equiv 1 \quad \bmod 2^{u+\tau(k)} \\
d & \equiv e^{-1} \quad \bmod 2^{u+\tau(k)} .
\end{aligned}
$$

Com a equação acima podemos asseverar que os $u+\tau(k)$ LSB de $d$ são dados por $e^{-1} \bmod 2^{u+\tau(k)}$, portanto podemos corrigir esses bits em $\tilde{d}$.

$$
\tilde{d}[j]=\left(e^{-1} \bmod 2^{u+\tau(k)}\right)[j] \text { para } 0 \leq j \leq u+\tau(k)
$$

As mesmas definições são aplicadas para a correção de alguns LSB dos expoentes de decriptação $d_{i}$, portanto temos que

$$
\begin{array}{cr}
2 \mid\left(r_{i}-1\right) & \text { Devido a que } r_{i} \text { é primo } \\
2^{1+\tau\left(k_{i}\right)} \mid k_{i}\left(r_{i}-1\right) & \text { Visto que } 2^{\tau\left(k_{i}\right)} \mid k_{i} \\
2^{1+\tau\left(k_{i}\right)} \mid e d_{i}-1 & \text { Pela equação }(3.3)
\end{array}
$$

a qual pode ser expressada como

$$
\begin{aligned}
e d_{i}-1 & \equiv 0 \quad \bmod 2^{1+\tau\left(k_{i}\right)} \\
e d_{i} & \equiv 1 \quad \bmod 2^{1+\tau\left(k_{i}\right)} \\
d_{i} & \equiv e^{-1} \quad \bmod 2^{1+\tau\left(k_{i}\right)} .
\end{aligned}
$$

Em outras palavras, podemos corrigir que os $1+\tau\left(k_{i}\right)$ LSB de $\tilde{d}_{i}$, os quais estão dados por $e^{-1}$ $\bmod 2^{1+\tau\left(k_{i}\right)}$.

$$
\tilde{d}_{i}[j]=\left(e_{i}^{-1} \bmod 2^{1+\tau\left(k_{i}\right)}\right)[j] \text { para } 0 \leq j \leq u+\tau(k) \text { e } 1 \leq i \leq u
$$

Os valores de $e^{-1} \bmod 2^{u+\tau(k)}$ e $e_{i}^{-1} \bmod 2^{1+\tau\left(k_{i}\right)}$ para $1 \leq i \leq u$, podem ser calculados facilmente usando o algoritmo de Euclides-Estendido. Com essas correções feitas procedemos a explicar as dependências dos bits de cada variável de $s k$.

\subsection{Lema de Hensel - Geração das equações}

Para analisar as dependências com relação aos bits das variáveis da chave secreta RSA $s k$ explicamos o lema a seguir.

Lema 1 (Lema de Hensel para multivariáveis [HS09]). Uma raiz $r=\left(r_{1}, r_{2}, \ldots, r_{u}\right)$ do polinômio $f\left(x_{1}, x_{2}, \ldots, x_{u}\right) \bmod \pi^{j}$ pode ser usada para gerar uma raiz $r+b \bmod \pi^{j+1}$ se $b=\left(b_{1} \pi^{j}, b_{2} \pi^{j}, \ldots, b_{u} \pi^{j}\right)$, $0 \leq b_{i} \leq \pi-1$ é uma solução para a equação

$$
f(r+b)=f(r)+\sum_{i} b_{i} \pi^{j} f_{x_{i}}(r) \equiv 0 \quad\left(\bmod \pi^{j+1}\right) .
$$

(onde, $f_{x_{j}}$ é a derivada parcial de $f$ com relação a $x_{j}$ )

Para a análise vamos usar o valor para $\pi=2$ o qual vai permitir a reconstrução da chave secreta $s k$ bit a bit desde o bit menos significativo até o mais significativo. Para a análise definimos os 
seguintes polinômios

$$
\begin{aligned}
f\left(x_{1}, x_{2}, \ldots, x_{u}\right) & =N-\prod_{i=1}^{u} x_{i} \\
f\left(x_{1}, x_{2}, \ldots, x_{u}, y\right) & =e y-1-k \prod_{i=1}^{u}\left(x_{i}-1\right) \\
f\left(x_{i}, y_{i}\right) & =e y_{i}-1-k_{i}\left(x_{i}-1\right) \quad \text { para } 1 \leq i \leq u
\end{aligned}
$$

obtidos a partir das equações (3.1), (3.2) e (3.3) do RSA. Onde a raiz deles está dada por

$$
r\left\langle y, x_{1}, x_{2}, y_{1}, y_{2},\left\langle x_{3}, y_{3}\right\rangle, \ldots,\left\langle x_{u}, y_{u}\right\rangle\right\rangle=\left\langle d, r_{1}, r_{2}, d_{1}, d_{2},\left\langle r_{3}, d_{3}\right\rangle, \ldots,\left\langle r_{u}, d_{u}\right\rangle\right\rangle .
$$

A seguir vamos fazer a análise do Lema 1 para cada um desses polinômios.

Analisando o Polinômio (3.13), definimos uma raiz $\left(r_{1}^{\prime}, r_{2}^{\prime}, \ldots, r_{u}^{\prime}\right)$ do polinômio $f\left(x_{1}, x_{2}, \ldots, x_{u}\right)$ $\left(\bmod 2^{j}\right)$ onde a partir dela geramos uma raiz equivalente para $f\left(x_{1}, x_{2}, \ldots, x_{u}\right)\left(\bmod 2^{j+1}\right)$. Para isso, uns dos requisitos são as derivadas do respectivo polinômio.

$$
f_{x_{i}}\left(x_{1}, x_{2} \ldots, x_{u}\right)=-\frac{\prod_{l=1}^{u} x_{l}}{x_{i}} \text { para } 1 \leq i \leq u
$$

Usando a Lema 1 para o polinômio $f\left(x_{1}, x_{2}, \ldots, x_{u}\right)$ podemos definir que os valores $b_{i} \in\{0,1\}$ portanto representam os valores dos bits $r_{i}[j]$, onde temos que

$$
\begin{array}{rrr}
f\left(r_{1}^{\prime}, r_{2}^{\prime}, \ldots, r_{u}^{\prime}\right)+\sum_{i=1}^{u} r_{i}[j] 2^{j} f_{x_{i}}\left(r_{1}^{\prime}, r_{2}^{\prime}, \ldots, r_{u}^{\prime}\right) \equiv 0 & \left(\bmod 2^{j+1}\right) & \text { Substituindo } b_{i} \text { por } r_{i}[j] \\
f\left(r_{1}^{\prime}, r_{2}^{\prime}, \ldots, r_{u}^{\prime}\right)-\sum_{i=1}^{u} r_{i}[j] 2^{j} \frac{\prod_{l=1}^{u} r_{l}^{\prime}}{r_{i}^{\prime}} \equiv 0 & \left(\bmod 2^{j+1}\right) & \text { Substituindo } f_{x_{i}}\left(r_{1}^{\prime}, r_{2}^{\prime}, \ldots, r_{u}^{\prime}\right) .
\end{array}
$$

Observa-se que o valor $r_{i}[j] 2^{j} \frac{\prod_{l=1}^{u} r_{l}^{\prime}}{r_{i}^{\prime}} \bmod 2^{j+1}=r_{i}[j] 2^{j}$ já que $\frac{\prod_{l=1}^{u} r_{l}^{\prime}}{r_{i}^{\prime}}$ é um número ímpar, portanto temos

$$
\begin{aligned}
f\left(r_{1}^{\prime}, r_{2}^{\prime}, \ldots, r_{u}^{\prime}\right)-\sum_{i=1}^{u} r_{i}[j] 2^{j} & \equiv 0 \quad\left(\bmod 2^{j+1}\right) \\
N-\prod_{i=1}^{u} r_{i}^{\prime} & \equiv \sum_{i=1}^{u} r_{i}[j] 2^{j} \quad\left(\bmod 2^{j+1}\right) \quad \text { Substituindo } f\left(r_{1}^{\prime}, r_{2}^{\prime}, \ldots, r_{u}^{\prime}\right) .
\end{aligned}
$$

Se analisamos a dependência dos bits na equação acima podemos determinar que

$$
\left(N-\prod_{i=1}^{u} r_{i}^{\prime}\right)[j] \equiv \sum_{i=1}^{u} r_{i}[j] \quad(\bmod 2)
$$

já que $N-\prod_{i=1}^{u} r_{i}^{\prime} \equiv 0(\bmod 2)^{j}$. Com o qual podemos definir que as raízes para $f\left(x_{1}, x_{2}, \ldots, x_{u}\right)$ $\left(\bmod 2^{j+1}\right)$ são dadas por $\left(r_{1}^{\prime}+2^{j} r_{1}[j], r_{2}^{\prime}+2^{j} r_{2}[j], \ldots, r_{u}^{\prime}+2^{j} r_{u}[j]\right)$ onde cada $r_{i}[j]$ pode ter 2 valores (0 ou 1), mas esses valores devem cumprir com a Equação (3.16) de Hensel.

Com relação ao segundo Polinômio (3.14), definimos uma raiz $\left(r_{1}^{\prime}, r_{2}^{\prime}, \ldots, r_{u}^{\prime}, d^{\prime}\right)$ do polinômio $f\left(x_{1}, x_{2}, \ldots, x_{u}, y\right)\left(\bmod 2^{j}\right)$, onde podemos observar que o valor de $r_{i}^{\prime}$ é multiplicado por $k$ fazendo que os bits de $r_{i}^{\prime}$ sejam deslocados á esquerda em $\tau(k)$ posições já que $2^{\tau(k)} \mid k$, portanto os valores $r_{i}^{\prime}$ tem um tamanho máximo de $j-\tau(k)-1$ bits, assim que quando calculamos as raízes para o polinômio $f\left(x_{1}, x_{2}, \ldots, x_{u}, y\right)\left(\bmod 2^{j}\right)$ simplesmente estamos determinando os bits $r_{i}[j-\tau(k)]$ para $1 \leq i \leq u$, mas para $d^{\prime}$ estamos determinado o valor de $d[j]$ já que não existe deslocamento para ele. Assim que para usar o lema de Hensel devemos calcular as derivadas do Polinômio (3.14), as 
quais são definidas a seguir:

$$
\begin{array}{rlrl}
f_{x_{i}}\left(x_{1}, x_{2}, \ldots, x_{u}, y\right) & =-k \frac{\prod_{l=1}^{u}\left(x_{l}-1\right)}{x_{i}-1} & \text { para } 1 \leq i \leq u \\
f_{y}\left(x_{1}, x_{2}, \ldots, x_{u}, y\right) & =e & &
\end{array}
$$

Com as definições anteriores podemos estabelecer com o Lema 1 que

$f\left(r_{1}^{\prime}, r_{2}^{\prime}, \ldots, r_{u}^{\prime}, d^{\prime}\right)+\sum_{i=1}^{u} r_{i}[j-\tau(k)] 2^{j-\tau(k)} f_{x_{i}}\left(r_{1}^{\prime}, \ldots, r_{u}^{\prime}, d^{\prime}\right)+d[j] 2^{j} f_{y}\left(r_{1}^{\prime}, \ldots, r_{u}^{\prime}, d^{\prime}\right) \equiv 0 \quad\left(\bmod 2^{1+j}\right)$,

mas nós queremos uma relação com o bit $r_{i}[j]$ para assim poder obter uma relação direta com a Equação (3.16) de Hensel, portanto podemos redefinir que $\left(r_{1}^{\prime}, r_{2}^{\prime}, \ldots, r_{u}^{\prime}, d^{\prime}\right)$ é uma raiz de $f\left(x_{1}, x_{2}, \ldots, x_{u}, y\right)$ $\left(\bmod 2^{j+\tau(k)}\right)$ e para assim analisando o Lema 1 obter seu equivalente com relação a $f\left(x_{1}, x_{2}, \ldots, x_{u}, y\right)$ $\left(\bmod 2^{1+j+\tau(k)}\right)$. Portanto com o Lema 1 para o polinômio (3.14) do RSA temos

$f\left(r_{1}^{\prime}, \ldots, r_{u}^{\prime}, d^{\prime}\right)+\sum_{i=1}^{u} r_{i}[j] 2^{j} f_{x_{i}}\left(r_{1}^{\prime}, \ldots, r_{u}^{\prime}, d^{\prime}\right)+d[j+\tau(k)] 2^{j+\tau(k)} f_{y}\left(r_{1}^{\prime}, \ldots, r_{u}^{\prime}, d^{\prime}\right) \equiv 0 \quad\left(\bmod 2^{1+j+\tau(k)}\right)$.

Onde podemos afirmar que $\sum_{i=1}^{u} r_{i}[j] 2^{j} f_{x_{i}}\left(r_{1}^{\prime}, \ldots, r_{u}^{\prime}, d^{\prime}\right) \equiv 0\left(\bmod 2^{1+j+\tau(k)}\right)$.

$$
\begin{array}{cr}
2^{u-1} \mid-\frac{\prod_{l=1}^{u}\left(r_{l}^{\prime}-1\right)}{r_{i}^{\prime}-1} & \text { Visto que } r_{l}^{\prime} \text { e } r_{i}^{\prime} \text { são ímpares } \\
2^{u-1+\tau(k)} \mid-k \frac{\prod_{l=1}^{u}\left(r_{l}^{\prime}-1\right)}{r_{i}^{\prime}-1} & \text { Visto que } 2^{\tau(k)} \mid k \\
2^{u-1+\tau(k)} \mid f_{x_{i}}\left(r_{1}^{\prime}, r_{2}^{\prime}, \ldots, r_{u}^{\prime}, d^{\prime}\right) & \text { Definição de } f_{x_{i}}\left(x_{1}, x_{2}, \ldots, x_{u}, y\right) \\
2^{u-1+\tau(k)+j} \mid 2^{j} f_{x_{i}}\left(r_{1}^{\prime}, r_{2}^{\prime}, \ldots, r_{u}^{\prime}, d^{\prime}\right) & \text { Multiplicando por } 2^{j} \\
2^{1+\tau(k)+j} \mid 2^{j} f_{x_{i}}\left(r_{1}^{\prime}, r_{2}^{\prime}, \ldots, r_{u}^{\prime}, d^{\prime}\right) & \text { Visto que } u \geq 2
\end{array}
$$

Portanto temos

$$
f\left(r_{1}^{\prime}, r_{2}^{\prime}, \ldots, r_{u}^{\prime}, d^{\prime}\right)+d[j+\tau(k)] 2^{j+\tau(k)} f_{y}\left(r_{1}^{\prime}, r_{2}^{\prime}, \ldots, r_{u}^{\prime}, d^{\prime}\right) \equiv 0 \quad\left(\bmod 2^{1+j+\tau(k)}\right)
$$

onde $d[j+\tau(k)] 2^{j+\tau(k)} f_{y}\left(r_{1}^{\prime}, r_{2}^{\prime}, \ldots, r_{u}^{\prime}, d^{\prime}\right) \equiv d[j+\tau(k)] 2^{j+\tau(k)}\left(\bmod 2^{1+j+\tau(k)}\right)$ já que $f_{y}\left(r_{1}^{\prime}, r_{2}^{\prime}, \ldots, r_{u}^{\prime}, d^{\prime}\right)=$ $e$ é impar, assim que

$$
\begin{aligned}
f\left(r_{1}^{\prime}, r_{2}^{\prime}, \ldots, r_{u}^{\prime}, d^{\prime}\right)+d[j+\tau(k)] 2^{j+\tau(k)} & \equiv 0 \quad\left(\bmod 2^{1+j+\tau(k)}\right) \\
e d^{\prime}-1-k \prod_{i=1}^{u}\left(r_{i}^{\prime}-1\right)+d[j+\tau(k)] 2^{j+\tau(k)} & \equiv 0 \quad\left(\bmod 2^{1+j+\tau(k)}\right) \quad \text { Substituindo } f\left(r_{1}^{\prime}, r_{2}^{\prime}, \ldots, r_{u}^{\prime}, d^{\prime}\right)
\end{aligned}
$$

e como sabemos que $\left(r_{1}^{\prime}, r_{2}^{\prime}, \ldots, r_{u}^{\prime}, d^{\prime}\right)$ é raiz do polinômio $f\left(x_{1}, x_{2}, \ldots, x_{u}, y\right)(\bmod 2)^{j+\tau(k)}$ podemos estabelecer que a relação de bits é dada por

$$
\left(e d^{\prime}-1-k \prod_{i=1}^{u}\left(r_{i}^{\prime}-1\right)\right)[j+\tau(k)]+d[j+\tau(k)] \equiv 0 \quad(\bmod 2) .
$$

onde a equação acima pode ser expressada por

$$
\left(e d^{\prime}-1-k \prod_{i=1}^{u}\left(r_{i}^{\prime}-1\right)\right)[j+\tau(k)] \equiv d[j+\tau(k)] \quad(\bmod 2)
$$

já que estamos em módulo 2. Portanto podemos definir que as raízes do polinômio $f\left(x_{1}, x_{2}, \ldots, x_{u}, y\right)$ 
$\left(\bmod 2^{1+j+\tau(k)}\right)$ estão dadas por $\left(r_{1}^{\prime}+2^{j} r_{1}[j], r_{2}^{\prime}+2^{j} r_{2}[j], \ldots, r_{u}^{\prime}+2^{j} r_{u}[j], d^{\prime}+2^{j+\tau(k)} d[j+\tau(k)]\right)$ onde cada $r_{i}[j]$ pode ter dois valores (0 ou 1 ) mas o valor de $d[j+\tau(k)]$ deve cumprir com a Equação (3.17) de Hensel.

Por último fazemos a análise para o Polinômio (3.15)

$$
f\left(x_{i}, y_{i}\right)=e y_{i}-1-k_{i}\left(x_{i}-1\right) \quad \text { para } 1 \leq i \leq u
$$

onde $\left(r_{i}^{\prime}, d_{i}^{\prime}\right)$ é raiz de $f\left(x_{i}, y_{i}\right)\left(\bmod 2^{j+\tau\left(k_{i}\right)}\right)$. Para aproveitar os bits $r_{i}[j]$, o valor de $r^{\prime}$ está conformado por um total de $j-1$ bits já que seu valor é multiplicado por $k_{i}$ fazendo que os bits de $r_{i}^{\prime}$ sejam deslocados á esquerda em $\tau\left(k_{i}\right)$ posições (já que $\left.2^{\tau\left(k_{i}\right)} \mid k_{i}\right)$, portanto quando calculamos as raízes para o polinômio $f\left(x_{i}, y_{i}\right)\left(\bmod 2^{1+j+\tau\left(k_{i}\right)}\right)$ estamos calculando o bit $r_{i}[j]$ em relação a $r_{i}^{\prime}$, mas para $d_{i}^{\prime}$ estamos determinado o valor de $d_{i}\left[j+\tau\left(k_{i}\right)\right]$. Portanto usando o Lema 1 temos que

$$
f\left(r_{i}^{\prime}, d_{i}^{\prime}\right)+r[j] 2^{j} f_{x_{i}}\left(r_{i}^{\prime}, d_{i}^{\prime}\right)+d_{i}\left[j+\tau\left(k_{i}\right)\right] 2^{j+\tau\left(k_{i}\right)} f_{y_{i}}\left(r_{i}^{\prime}, d_{i}^{\prime}\right) \equiv 0 \quad\left(\bmod 2^{1+j+\tau\left(k_{i}\right)}\right)
$$

onde $f_{x_{i}}\left(x_{i}, y_{i}\right)=-k_{i}$ e $f_{y_{i}}\left(x_{i}, y_{i}\right)=e$.

$$
f\left(r_{i}^{\prime}, d_{i}^{\prime}\right)-r[j] 2^{j} k_{i}+d_{i}\left[j+\tau\left(k_{i}\right)\right] 2^{j+\tau\left(k_{i}\right)} e \equiv 0 \quad\left(\bmod 2^{1+j+\tau\left(k_{i}\right)}\right)
$$

Na equação acima temos que $k_{i} 2^{-\tau\left(k_{i}\right)}$ e $e$ são ímpares e onde os valores $r[j] 2^{j} k_{i}, d_{i}\left[j+\tau\left(k_{i}\right)\right] 2^{j+\tau\left(k_{i}\right)}$ e $f\left(r_{i}^{\prime}, d_{i}^{\prime}\right)$ são múltiplos de $2^{j+\tau\left(k_{i}\right)}$. portanto podemos estabelecer que a relação de bits é dado por

$$
\left(1+k_{i}\left(r_{i}^{\prime}-1\right)-e d_{i}^{\prime}\right)\left[j+\tau\left(k_{i}\right)\right]-r[j]+d_{i}\left[j+\tau\left(k_{i}\right)\right] \equiv 0 \quad(\bmod 2) \quad \text { para } 1 \leq i \leq u
$$

onde essas equações acima por estar em módulo 2 podem ser reescritas como

$$
\left(1+k_{i}\left(r_{i}^{\prime}-1\right)-e d_{i}^{\prime}\right)\left[j+\tau\left(k_{i}\right)\right] \equiv r[j]+d_{i}\left[j+\tau\left(k_{i}\right)\right] \quad(\bmod 2) \quad \text { para } 1 \leq i \leq u .
$$

Portanto temos que $\left(r_{i}^{\prime}+2^{j} r_{i}[j], d_{i}^{\prime}+2^{j+\tau\left(k_{i}\right)} d_{i}\left[j+\tau\left(k_{i}\right)\right]\right)$ é uma raiz do $f\left(x_{i}, y_{i}\right)\left(\bmod 2^{j+\tau\left(k_{i}\right)+1}\right)$ só se os valores atribuídos para $r_{i}[j]$ e $d_{i}\left[j+\tau\left(k_{i}\right)\right]$ cumprem com a Equação (3.18) de Hensel.

Portanto podemos observar que as relações ou dependências que existem entre os bits são dadas nas seguintes equações

$$
\begin{aligned}
& \left(N-\prod_{i=1}^{u} r_{i}^{\prime}\right)[j] \equiv \sum_{i=1}^{u} r_{i}[j] \\
& \left(e d^{\prime}-1-k \prod_{i=1}^{u}\left(r_{i}^{\prime}-1\right)\right)[j+\tau(k)] \equiv d[j+\tau(k)] \quad(\bmod 2) \\
& \left(e d_{i}^{\prime}-1-k\left(r_{i}^{\prime}-1\right)\right)\left[j+\tau\left(k_{i}\right)\right] \equiv r_{i}[j]+d_{i}\left[j+\tau\left(k_{i}\right)\right] \quad(\bmod 2) \quad \text { para } 1 \leq i \leq u
\end{aligned}
$$
Com relação a primeira equação podemos afirmar que o valor do bit $j$ de uns dos primos está
relacionado com os bits $j$ dos outros primos. Também observamos que o bit $d[j+\tau(k)]$ não está relacionado diretamente com nenhum dos bits das outras variáveis de $s k$ e por último temos uma relação entre os bits $r_{i}[j]$ e $d_{i}\left[j+\tau\left(k_{i}\right)\right]$ para $1 \leq i \leq u$.

\subsection{Algoritmo e seu comportamento}

Com as definições da Seção 3.3 vamos definir $\operatorname{root}[j-1]$ como um conjunto de raízes do tipo $\left\langle d^{\prime}, r_{1}^{\prime}, r_{2}^{\prime}, d_{1}^{\prime}, d_{2}^{\prime},\left\langle r_{3}^{\prime}, d_{3}^{\prime}\right\rangle, \ldots,\left\langle r_{u}^{\prime}, d_{u}^{\prime}\right\rangle\right\rangle$, a qual é a raiz dos seguintes polinômios RSA

$$
\begin{array}{rllll}
f\left(r_{1}^{\prime}, r_{2}^{\prime}, \ldots, r_{u}^{\prime}\right) & \equiv N-\prod_{i=1}^{u} r_{i}^{\prime} & \equiv & (\bmod 2)^{j} & \\
f\left(r_{1}^{\prime}, r_{2}^{\prime}, \ldots, r_{u}^{\prime}, d^{\prime}\right) & \equiv e d^{\prime}-1-k \prod_{i=1}^{u}\left(r_{i}^{\prime}-1\right) & \equiv & 0 & (\bmod 2)^{j+\tau(k)} \\
f\left(r_{i}^{\prime}, d_{i}^{\prime}\right) & \equiv e d_{i}^{\prime}-1-k_{i}\left(r_{i}^{\prime}-1\right) & \equiv 0 & (\bmod 2)^{j+\tau\left(k_{i}\right)} & \text { para } 1 \leq i \leq u .
\end{array}
$$


E usando os resultados da seção anterior, podemos gerar todas as possíveis raízes que pertencem a $\operatorname{root}[j]$ a partir de cada raiz $\left\langle d^{\prime}, r_{1}^{\prime}, r_{2}^{\prime}, d_{1}^{\prime}, d_{2}^{\prime},\left\langle r_{3}^{\prime}, d_{3}^{\prime}\right\rangle, \ldots,\left\langle r_{u}^{\prime}, d_{u}^{\prime}\right\rangle\right\rangle \in \operatorname{root}[j-1]$, onde os valores de uma raiz $\left\langle d^{*}, r_{1}^{*}, r_{2}^{*}, d_{1}^{*}, d_{2}^{*},\left\langle r_{3}^{*}, d_{3}^{*}\right\rangle, \ldots,\left\langle r_{u}^{*}, d_{u}^{*}\right\rangle\right\rangle \in \operatorname{root}[j]$ está definida por

$$
\begin{aligned}
& d^{*}=d^{\prime}+2^{j+\tau(k)} d[j+\tau(k)] \\
& r_{i}^{*}=r_{i}^{\prime}+2^{j} r_{i}[j] \quad \text { para } 1 \leq i \leq u \\
& d_{i}^{*}=d_{i}^{\prime}+2^{j+\tau\left(k_{i}\right)} d_{i}\left[j+\tau\left(k_{i}\right)\right] \quad \text { para } 1 \leq i \leq u
\end{aligned}
$$

onde os valores para $r_{i}[j], d_{i}\left[j+\tau\left(k_{i}\right)\right]$ e $d[j+\tau(k)]$ podem ter dois valores (0 ou 1$)$, mas esses valores devem cumprir com as seguintes equivalências

$$
\begin{aligned}
& \left(N-\prod_{i=1}^{u} r_{i}^{\prime}\right)[j] \equiv \sum_{i=1}^{u} r_{i}[j] \\
& \left(e d^{\prime}-1-k \prod_{i=1}^{u}\left(r_{i}^{\prime}-1\right)\right)[j+\tau(k)] \equiv d[j+\tau(k)] \\
& \left(e d_{i}^{\prime}-1-k\left(r_{i}^{\prime}-1\right)\right)\left[j+\tau\left(k_{i}\right)\right] \equiv r_{i}[j]+d_{i}\left[j+\tau\left(k_{i}\right)\right] \quad(\bmod 2) \quad \text { para } 1 \leq i \leq u
\end{aligned}
$$

obtidas pela aplicação do lema de Hensel às equações RSA.

O algoritmo de reconstrução simplesmente é um algoritmo do tipo voraz, em outras palavras, geramos todas as raízes possíveis para os polinômios RSA. Em outras palavras, para cada raiz em $\operatorname{root}[j-1]$ geramos todas as possíveis raízes para o root $[j]$, e tudo isso tendo em consideração que alguns bits em

$$
\left\langle d[j+\tau(k)], r_{1}[j], r_{2}[j], d_{1}\left[j+\tau\left(k_{1}\right)\right], d_{2}\left[j+\tau\left(k_{2}\right)\right],\left\langle r_{3}[j], d_{3}\left[j+\tau\left(k_{3}\right)\right]\right\rangle, \ldots,\left\langle r_{u}[j], d_{u}\left[j+\tau\left(k_{u}\right)\right]\right\rangle\right\rangle
$$

são conhecidos (devido à fração $\delta$ de bits corretos em $\tilde{s k}$ de $s k$ ).

$\mathrm{O}$ algoritmo começa com o conjunto root $[0]$ conformado por um único elemento

$$
\operatorname{root}[0]=\left[\left\langle d^{\prime}, r_{1}^{\prime}, r_{2}^{\prime}, d_{1}^{\prime}, d_{2}^{\prime},\left\langle r_{3}^{\prime}, d_{3}^{\prime}\right\rangle, \ldots,\left\langle r_{u}^{\prime}, d_{u}^{\prime}\right\rangle\right\rangle\right]
$$

onde $\left\langle d^{\prime}, r_{1}^{\prime}, r_{2}^{\prime}, d_{1}^{\prime}, d_{2}^{\prime},\left\langle r_{3}^{\prime}, d_{3}^{\prime}\right\rangle, \ldots,\left\langle r_{u}^{\prime}, d_{u}^{\prime}\right\rangle\right\rangle$ é raiz dos polinômios

$$
\begin{aligned}
& f\left(r_{1}^{\prime}, r_{2}^{\prime}, \ldots, r_{u}^{\prime}\right) \equiv N-\prod_{i=1}^{u} r_{i}^{\prime} \quad \equiv 0 \quad(\bmod 2) \\
& f\left(r_{1}^{\prime}, r_{2}^{\prime}, \ldots, r_{u}^{\prime}, d^{\prime}\right) \equiv e d^{\prime}-1-k \prod_{i=1}^{u}\left(r_{i}^{\prime}-1\right) \equiv 0 \quad(\bmod 2)^{1+\tau(k)} \\
& f\left(r_{i}^{\prime}, d_{i}^{\prime}\right) \equiv e d_{i}^{\prime}-1-k_{i}\left(r_{i}^{\prime}-1\right) \equiv 0 \quad(\bmod 2)^{1+\tau\left(k_{i}\right)} \quad \text { para } 1 \leq i \leq u .
\end{aligned}
$$

E sabemos que: $N$ é ímpar $(N \bmod 2=1) ; 2^{1+\tau(k)} \mid k \prod_{i=1}^{u}\left(r_{i}^{\prime}-1\right)$ e $2^{1+\tau\left(k_{i}\right)} \mid k_{i} \prod_{i=1}^{u}\left(r_{i}^{\prime}-1\right)$ para $1 \leq i \leq u$ (visto na Seção 3.2). Portanto temos que

$$
\begin{aligned}
f\left(r_{1}^{\prime}, r_{2}^{\prime}, \ldots, r_{u}^{\prime}\right) & \equiv \prod_{i=1}^{u} r_{i}^{\prime} \equiv 1 \quad(\bmod 2) \\
f\left(r_{1}^{\prime}, r_{2}^{\prime}, \ldots, r_{u}^{\prime}, d^{\prime}\right) & \equiv e d^{\prime} \equiv 1 \quad(\bmod 2)^{1+\tau(k)} \\
f\left(r_{i}^{\prime}, d_{i}^{\prime}\right) & \equiv e d_{i}^{\prime} \equiv 1 \quad(\bmod 2)^{1+\tau\left(k_{i}\right)} \quad \text { para } 1 \leq i \leq u .
\end{aligned}
$$

Onde os valores para $r_{i}^{\prime}$ são dados por $r_{i}^{\prime}=1$ para $1 \leq i \leq u$, o valor de $d^{\prime}$ é dado por $d^{\prime}=e^{-1}$ $\bmod 2^{1+\tau(k)}$ e por último os valores para $d_{i}^{\prime}$ são dados por $d_{i}^{\prime}=e^{-1} \bmod 2^{1+\tau\left(k_{i}\right)}$ para $1 \leq i \leq u$. Os valores de $d^{\prime}$ e $d_{i}^{\prime}$ podem ser calculados usando o algoritmo de Euclides-Estendido. Portanto podemos concluir que o conjunto root [0] tem um único elemento e é conhecido. Existe outro método para calcular o único elemento do conjunto root[0], o qual é usando alguns conceitos do lema de Hensel, de acordo à Seção 3.3 sabemos que os valores de $r_{i}^{\prime}$ para o conjunto root [0] estão conformados só por um bit, o valor de $d^{\prime}$ está conformado por $1+\tau(k)$ bits e os valores $d_{i}^{\prime}$ estão conformados por $1+\tau\left(k_{i}\right)$ bits (para $1 \leq i \leq u$ ). Onde esses bits são conhecidos e foram corrigidos em $\tilde{s k}$ na Seção 
3.2 portanto temos que

$$
\begin{aligned}
& r_{i}^{\prime}=\tilde{r}_{i} \quad \bmod 2 \text { para } 1 \leq i \leq u \\
& d^{\prime}=\tilde{d} \quad \bmod 2^{1+\tau(k)} \\
& d_{i}^{\prime}=\tilde{d}_{i} \quad \bmod 2^{1+\tau\left(k_{i}\right)} \text { para } 1 \leq i \leq u .
\end{aligned}
$$

O algoritmo de reconstrução gera todas as possíveis raízes para o conjunto root [1] a partir da única raiz de root [0] e para cada raiz do root[1] vai gerar todas suas possíveis raízes para o root [2] e assim sucessivamente até chegar ao conjunto $\operatorname{root}\left[\frac{n}{u}\right]$.

$$
\forall r_{j-1} \in \operatorname{root}[j-1] \Rightarrow r_{j} \in \operatorname{root}[j] \text { para } 1 \leq j \leq \frac{n}{u}
$$

Onde uma das suas raízes do conjunto root $\left[\frac{n}{u}\right]$ contém os valores dos fatores primos de $N$.

$$
\left\langle\tilde{d}, r_{1}, r_{2}, d_{1}, d_{2},\left\langle r_{3}, d_{3}\right\rangle, \ldots,\left\langle r_{u}, d_{u}\right\rangle\right\rangle \in \operatorname{root}\left[\frac{n}{u}\right]
$$

Isso devido aos valores de $r_{i}$ terem no máximo $\frac{n}{u}$ bits (já que $\mathrm{N}$ é balanceado). Com essas definições mostramos a implementação do algoritmo de reconstrução (veja o Algoritmo 2).

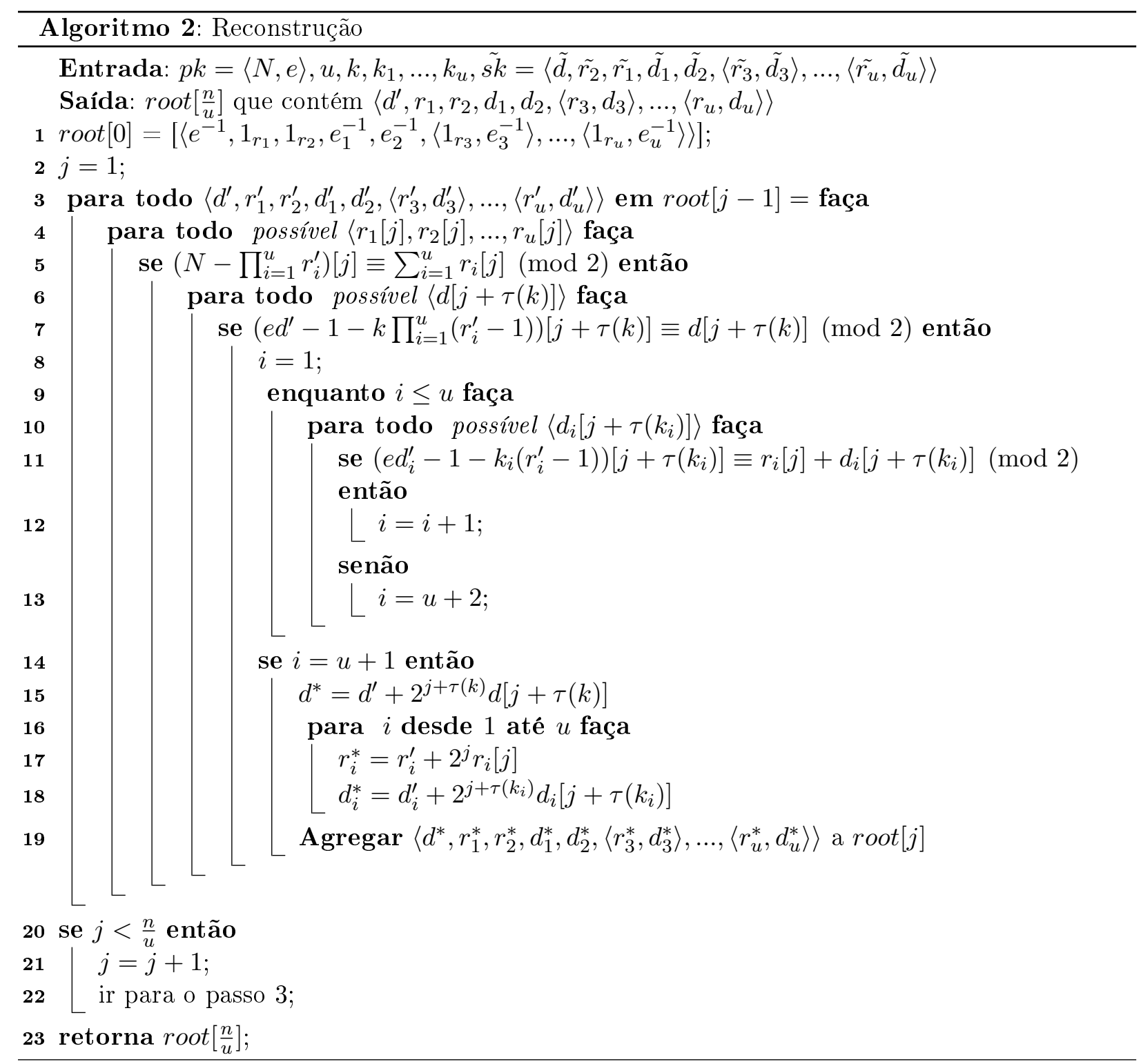


No Algoritmo 2 temos como dados de entrada: a chave pública $p k$, o valor de $u$, o valores precalculados das variáveis $k, k_{1}, k_{1}, \ldots, k_{u}$ e por último os bits conhecidos da chave secreta $\tilde{s k}$. Na linha 1 é declarado o conjunto root[0] para iniciar a reconstrução da chave secreta. Para cada raiz do conjunto root $[j-1]$ vamos gerar todos os possíveis valores dos bits $r_{i}[j], d_{i}\left[j+\tau\left(k_{i}\right)\right]$ e $d[j+\tau(k)]$ (sendo um único valor se o bit for conhecido em $\tilde{s k}$ ou dois se for desconhecido) especificado nas linhas 4, 6 e 10, depois verificamos as equações (3.16), (3.17) e (3.18) de Hensel nas linhas 5,7 e 11. Se todas a equivalências são verificadas então geramos a raiz que é depois acrescentada para o conjunto root $[j]$, isso definido nas linhas 14 até 19 . A saída do algoritmo é o conjunto root $\left[\frac{n}{u}\right]$ onde uma das suas raízes contém os fatores primos de $N$.

Sabemos que a partir de uma raiz de $\operatorname{root}[j-1]$ geramos um número determinado de raízes para $\operatorname{root}[j]$, e isso acontece para $1 \leq j \leq \frac{n}{u}$ portanto podemos definir que comportamento do algoritmo é igual a uma árvore onde cada nó é uma raiz. Ao final em root $\left[\frac{n}{u}\right]$ temos algumas raízes mas só uma delas contém os fatores primos de $N$ portanto essa raiz é definida como a raiz solução. Mas essa raiz solução tem sido formada a partir de várias raízes, mais especificamente é formada por uma raiz em cada conjunto root $[j]$ para $0 \leq j \leq \frac{n}{u}-1$, essas raízes são denominadas de raízes boas e as outras serão denotadas de incorretas (veja na Figura 3.1).

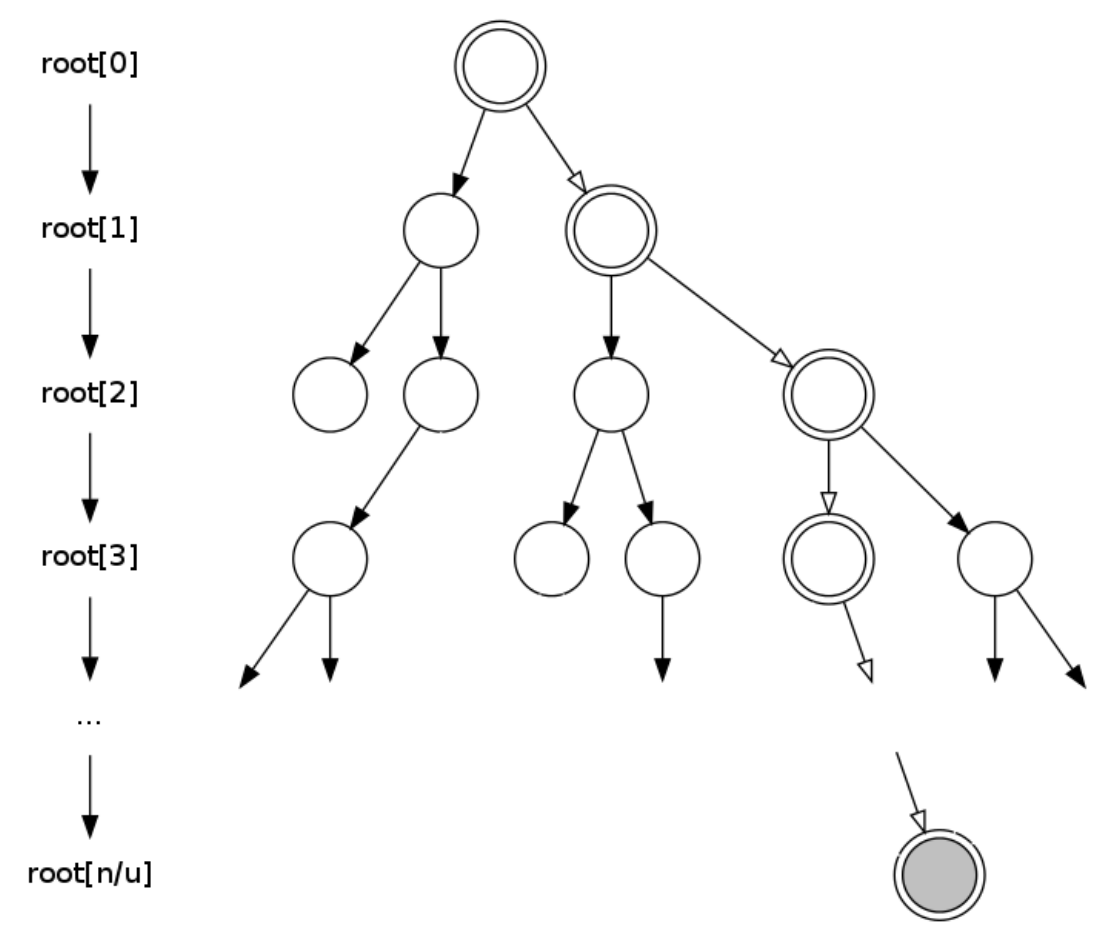

Figura 3.1: Representação do comportamento do algoritmo de reconstrução onde as circunferências: simples são as raízes incorretas, as duplas são as raízes boas e por último temos a circunferência dupla cinza, a qual é a representação da raiz correta.

Podemos pensar ingenuamente que a partir de uma raiz de $\operatorname{root}[j-1]$ geramos um total de $2^{2 u+1}$ raízes para o root $[j]$ já que temos um total de $2 u+1$ variáveis (bits desconhecidos) a achar e cada um delas pode ter dois possíveis valores ( 0 ou 1 ) mas o número de raízes é diminuído pelas relações que existe entre elas (equações (3.16), (3.17) e (3.18) de Hensel) e os bits que temos conhecimento em $\tilde{s k}$.

Para saber a complexidade do Algoritmo 2 não adianta calcular quantas raízes boas são analisadas já que em total temos $\frac{n}{u}$ raízes boas. Por tanto a complexidade do algoritmo depende completamente do comportamento das raízes incorretas, portanto devemos calcular o total de raízes incorretas que o algoritmo analisa. Além disso estamos falando de um algoritmo que sua função é reconstruir $s k$ a partir de uma fração $\delta$ de bits corretos em $\tilde{s k}$ portanto não é possível analisar com exatidão a complexidade mas é possível calcular a complexidade do algoritmo em relação a um valor esperado (veja a definição no Apêndice C.3.1) e a sua variância (veja a definição no Apêndice 
C.3.2). Para calcular esses valores definimos três novas variáveis aleatórias discretas (veja a definição no Apêndice C.1.1):

- $G$ : seja o número de raízes incorretas geradas por uma raiz boa.

- B: seja o número de raízes incorretas geradas por uma raiz incorreta.

- $X_{j}$ : seja o número de raízes incorretas do conjunto $\operatorname{root}[j]$.

\subsubsection{Complexidade do Algoritmo}

Para determinar a complexidade do algoritmo de reconstrução é preciso calcular o valor esperado e a variância do número de raízes incorretas analisadas na execução do algoritmo, portanto devemos determinar todos eventos que podem acontecer com sua respectiva probabilidade. Essa análise é feita com relação aos dois tipos de raízes que temos (boas e incorretas) para depois determinar o número de raízes incorretas analisadas em cada nível $\mathrm{j}$ (número de raízes no conjunto root $[j]$ ), e finalmente determinar o total de raízes incorretas analisadas em todo o algoritmo (que é o total de raízes incorretas desde o nível 0 até o nível $\frac{n}{u}$. Portanto procedemos a calcular o valor esperado de todas essas variáveis aleatórias discretas.

\section{Valor Esperado do Número de Raízes Incorretas Geradas por uma Raiz Boa $(\mathbb{E}[G])$}

Vamos supor que temos a única raiz boa de $\operatorname{root}[j-1]$ que é definida como

$$
r_{g}=\left\langle d^{\prime}, r_{1}^{\prime}, r_{2}^{\prime}, d_{1}^{\prime}, d_{2}^{\prime},\left\langle r_{3}^{\prime}, d_{3}^{\prime}\right\rangle, \ldots,\left\langle r_{u}^{\prime}, d_{u}^{\prime}\right\rangle\right\rangle
$$

e que a partir dela podemos calcular os valores para as variáveis $c_{1}$, $c_{2}$ e $c_{3_{i}}$ para $1 \leq i \leq u$ que são definidos por

$$
\begin{aligned}
c_{1} & =\left(N-\prod_{i=1}^{u} r_{i}^{\prime}\right)[j] \\
c_{2} & =\left(e d^{\prime}-1-k \prod_{i=1}^{u}\left(r_{i}^{\prime}-1\right)\right)[j+\tau(k)] \\
c_{3_{i}} & =\left(e d_{i}^{\prime}-1-k\left(r_{i}^{\prime}-1\right)\right)\left[j+\tau\left(k_{i}\right)\right] \quad \text { para } 1 \leq i \leq u .
\end{aligned}
$$

E pelas equações (3.16), (3.17) e (3.18) de Hensel podemos afirmar que

$$
\begin{aligned}
& c_{1} \equiv \sum_{i=1}^{u} r_{i}[j] \quad(\bmod 2) \\
& c_{2} \equiv d[j+\tau(k)] \quad(\bmod 2) \\
& c_{3_{i}} \equiv r_{i}[j]+d_{i}\left[j+\tau\left(k_{i}\right)\right] \quad(\bmod 2) \quad \text { para } 1 \leq i \leq u \text {. }
\end{aligned}
$$

Portanto para calcular o valor esperado de $G(\mathbb{E}[G])$ devemos analisar todos os possíveis eventos que podem acontecer com relação ao conhecimento ou desconhecimento dos bits

$$
\left\langle d[j+\tau(k)], r_{1}[j], r_{2}[j], d_{1}\left[j+\tau\left(k_{1}\right)\right], d_{2}\left[j+\tau\left(k_{2}\right)\right],\left\langle r_{3}[j], d_{3}\left[j+\tau\left(k_{3}\right)\right]\right\rangle, \ldots,\left\langle r_{u}[j], d_{u}\left[j+\tau\left(k_{u}\right)\right]\right\rangle\right\rangle
$$

onde o total de eventos analisados é dado pela multiplicação de estados (known e unknown) que pode ter cada bit, o qual resulta um total de $2^{2 u+1}$ eventos onde o valor de $u$ para nosso estudo não está definido $(u \geq 2)$.

No trabalho sobre fatoração de um inteiro multi-primo $N$ tendo só bits aleatórios dos seus fatores primos [TV] é declarado uma variável aleatória discreta $b_{u}$, esta variável $b_{u}$ define o número de bits desconhecidos no grupo de bits $\left\langle r_{1}[j], r_{2}[j], \ldots, r_{u}[j]\right\rangle$ facilitando o cálculo do número de raízes geradas. Poderíamos aplicar o mesmo conceito de $b_{u}$ mas resultá mais complicado, por exemplo: Vamos supor que temos o evento 


$$
\begin{aligned}
& \langle d[j+\tau(k)] \leftarrow \text { unknown } \\
& r_{1}[j] \leftarrow \text { unknown } \\
& r_{2}[j] \leftarrow \text { unknown } \\
& d_{1}\left[j+\tau\left(k_{1}\right)\right] \leftarrow \text { known } \\
& \left.d_{2}\left[j+\tau\left(k_{2}\right)\right] \leftarrow \text { unknown }\right\rangle
\end{aligned}
$$

no criptossistema RSA básico $(u=2)$ e segundo à definição de $b_{u}$ temos $b_{u}=2$, mas isso não é verdade já que o bit $r_{1}[j]$ pode ser calculado na Equação (3.18) para $i=1$ devido a que o bit $d_{1}\left[j+\tau\left(k_{1}\right)\right]$ é conhecido. O bit $r_{1}[j]$ vira de estado a known e muda o valor de $b_{u}$ a 1 , o qual essa troca de valor em $b_{u}$ representa uma diferença na quantidade de raízes geradas.

O processo de calcular o número de raízes geradas é dificultado completamente pela Equação (3.18) para $1 \leq i \leq u$, já que pelo conhecimento de alguns bits $d_{i}\left[j+\tau\left(k_{i}\right)\right.$ o valor de $b_{u}$ fica obsoleto. Para evitar essas mudanças de valor em $b_{u}$ vamos definir $B_{u}$, o qual é a variável aleatória discreta do número de bits desconhecidos no grupo de bits $\left\langle r_{1}[j], r_{2}[j], \ldots, r_{u}[j]\right\rangle$, mas depois de ter sido analisada a Equação (3.18) para $1 \leq i \leq u$. A definição de $B_{u}$ permite determinar o número de raízes incorretas geradas analisando só as duas primeiras equações (3.16) e (3.17), e deixa a Equação (3.18) (para $1 \leq i \leq u$ ) para determinar a função de probabilidade (Veja a definição no Apêndice C.2) com relação a $B_{u}$.

\section{Número de Raízes Incorretas Geradas por uma Raiz Boa}

Com os valores $c_{1}$ e $c_{2}$ já definidos e calculados procedemos a calcular o número de raízes geradas para $\operatorname{root}[j]$ a partir da raiz boa de $\operatorname{root}[j-1]$ e que é mostrado na Tabela 3.1.

\begin{tabular}{|c|c|c|}
\hline$B_{u}$ & $d[j+\tau(k)] \leftarrow$ known & $d[j+\tau(k)] \leftarrow$ unknown \\
\hline $1 \leq h \leq u$ & $2^{h-1}$ & $2^{h-1}$ \\
$h=0$ & 1 & 1 \\
\hline
\end{tabular}

Tabela 3.1: Número de raizes geradas a partir de uma raiz boa.

Na Tabela 3.1, a variável $h$ especifica o valor que pode tomar $B_{u}$, ou seja representa a quantidade de bits desconhecidos no grupo de bits $\left\langle r_{1}[j], r_{2}[j], \ldots, r_{u}[j]\right\rangle$ depois de analisar a Equação (3.18) para $1 \leq i \leq u$. Portanto segundo á Equação (3.16) com $1 \leq h \leq u$ bits desconhecidos temos uma equação módulo 2 com $h$ variáveis onde o total de raízes geradas está dado por $2^{h-1}$ (veja a Definição 17 ). No caso $h=0$ temos uma equivalência na Equação (3.16), portanto é gerado só uma única raiz para $\operatorname{root}[j]$ (devido a que uma raiz boa sempre gera outra única raiz boa). O conhecimento do bit $d[j+\tau(k)]$ não afeta ao número de raízes geradas pela Equação (3.16). Se o bit $d[j+\tau(k)]$ for desconhecido então atribuímos-lhe o valor de $c_{2}$, e se $d[j+\tau(k)]$ for conhecido então temos uma equivalência com relação á segunda Equação (3.17). Já calculado o número de raízes geradas a partir de uma raiz boa procedemos a calcular o número de raízes incorretas geradas a partir de uma raiz boa, o qual é só diminuir em 1 no número de raízes geradas (já que uma raiz boa sempre gera outra raiz boa e essa é única em cada nível) e é definido na Tabela 3.2.

\begin{tabular}{|c|c|c|}
\hline$B_{u}$ & $d[j+\tau(k)] \leftarrow$ known & $d[j+\tau(k)] \leftarrow$ unknown \\
\hline $1 \leq h \leq u$ & $2^{h-1}-1$ & $2^{h-1}-1$ \\
$h=0$ & 0 & 0 \\
\hline
\end{tabular}

Tabela 3.2: Número de raizes incorretas geradas a partir de uma raiz boa.

Com a Tabela 3.2 procedemos a calcular o valor esperado (ver fórmula no Apêndice C.3) de $G$, o qual é a somatória de todos os possíveis valores que pode ter $G$ multiplicado pela suas probabilidades 
e está definido por

$$
\mathbb{E}[G]=\sum_{h=1}^{u}\left(2^{h-1}-1\right) P\left(B_{u}=h\right)_{G}(P(d)+P(\bar{d}))
$$

onde $P(d)$ e $P(\bar{d})$ são as probabilidades do que o bit $d[j+\tau(k)]$ seja conhecido e desconhecido respectivamente e $P\left(B_{u}=h\right)_{G}$ é a probabilidade de $B_{u}$ ter o valor de $h$ em uma raiz boa. E que são explicadas a seguir.

\section{Probabilidades com relação a uma Raiz Boa}

As probabilidades estão determinadas pela porcentagem $\delta$ de bits corretos que temos em

$$
\tilde{s k}\left\langle\tilde{d}, \tilde{r_{1}}, \tilde{r_{2}}, \tilde{d}_{1}, \tilde{d}_{2},\left\langle\tilde{r_{3}}, \tilde{d}_{3}\right\rangle \ldots\left\langle\tilde{r_{u}}, \tilde{d_{u}}\right\rangle\right\rangle,
$$

portanto podemos definir as seguintes probabilidades

$$
\begin{aligned}
P(d) & =P(d[i+\tau(k)] \leftarrow \text { known })=\delta \\
P\left(r_{i}\right) & =P\left(r_{i}[j] \leftarrow \text { known }\right)=\delta \text { para } 1 \leq i \leq u \\
P\left(d_{i}\right) & =P\left(d_{i}\left[j+\tau\left(k_{i}\right)\right] \leftarrow \text { known }\right)=\delta \text { para } 1 \leq i \leq u
\end{aligned}
$$

com seus respectivos complementos

$$
\begin{aligned}
P(\bar{d}) & =P(d[i+\tau(k)] \leftarrow \text { unknown })=1-\delta \\
P\left(\overline{r_{i}}\right) & =P\left(r_{i}[j] \leftarrow \text { unknown }\right)=1-\delta \text { para } 1 \leq i \leq u \\
P\left(\overline{d_{i}}\right) & =P\left(d_{i}\left[j+\tau\left(k_{i}\right)\right] \leftarrow \text { unknown }\right)=1-\delta \text { para } 1 \leq i \leq u
\end{aligned}
$$

Definimos $P\left(r_{i}\right)_{G}$ e $P\left(\bar{r}_{i}\right)_{G}$ como as probabilidades do bit $r_{i}[j]$ ser conhecido ou desconhecido depois de ter analisado a Equação (3.18) para seu respectivo valor de $i$ em uma raiz boa. Portanto analisamos os estados do bit $r_{i}[j]$ em relação ao conhecimento ou desconhecimento do bit $d_{i}\left[j+\tau\left(k_{i}\right)\right]$. Para calcular o valor de $P\left(r_{i}\right)_{G}$ e $P\left(\bar{r}_{i}\right)_{G}$ vamos analisar todos os eventos que acontecem com relação à Equação (3.18), os quais são mostrados na Tabela 3.3.

\begin{tabular}{|c|c|c|}
\hline \multicolumn{2}{|c|}{ Eventos } & Estado de $r_{i}[j]_{\{G\}}=\{$ known, unknown $\}$ \\
\hline$r_{i}[j]$ & $d_{i}\left[j+\tau\left(k_{i}\right)\right]$ & $c_{3_{i}} \equiv r_{i}[j]+d_{i}\left[j+\tau\left(k_{i}\right)\right](\bmod 2)$ \\
\hline unknown & unknown & unknown \\
unknown & known & known \\
known & unknown & known \\
known & known & known \\
\hline
\end{tabular}

Tabela 3.3: Estado do bit $r_{i}[j]$ com relação ao estado do bit $d_{i}\left[j+\tau\left(k_{i}\right)\right]$ na raiz boa.

Procedemos a explicar a Tabela 3.3. Para o evento em que ambos bits $r_{i}[j]$ e $d_{i}\left[j+\tau\left(k_{i}\right)\right]$ são desconhecidos temos uma equação com duas variáveis dando um resultado de dois valores para o bit $r_{i}[j]$, portanto ele ainda é desconhecido. Para os outros eventos temos que o estado do bit $r_{i}[j]$ é known já que seu valor é conhecido ou pode ser calculado na Equação (3.18) para seu respectivo 
valor de $i$. Com todos esses eventos já analisados podemos determinar as seguintes probabilidades:

$$
\begin{aligned}
P\left(r_{i}\right)_{G}=P\left(r_{i}[j] \leftarrow \text { known }\right)_{G} & =P\left(\overline{r_{i}}\right) P\left(d_{i}\right)+P\left(r_{i}\right) P\left(\overline{d_{i}}\right)+P\left(r_{i}\right) P\left(d_{i}\right) \\
& =(1-\delta) \delta+\delta(1-\delta)+\delta \delta \\
& =2 \delta(1-\delta)+\delta^{2} \\
P\left(\overline{r_{i}}\right)_{G}=P\left(r_{i}[j] \leftarrow \text { unknown }\right)_{G} & =P\left(\overline{r_{i}}\right) P\left(\overline{d_{i}}\right) \\
& =(1-\delta)(1-\delta) \\
& =(1-\delta)^{2} .
\end{aligned}
$$

Quando a variável aleatória discreta $B_{u}$ tem o valor de $h$, estamos afirmando que temos $h$ bits desconhecidos no grupo de bits $\left\langle r_{1}[j], r_{2}[j], \ldots, r_{u}[j]\right\rangle$, portanto resulta que temos $u-h$ bits conhecidos. E a probabilidade de um bit $r_{i}[j]$ bit seja conhecido ou desconhecido não altera na probabilidade dos outros bits, portanto temos probabilidades independentes (veja a definição no Apêndice B.1.3) onde a probabilidade de um evento acontecer com $h$ bits desconhecidos e $u-h$ bits conhecidos é dada pela sua multiplicação. Mas esse evento acontece um número de vezes definido pelo número de combinações de $h$ elementos em um conjunto de $u$ elementos, o qual está definido por $\left(\begin{array}{l}u \\ h\end{array}\right)$ (veja o Teorema A.8). Portanto a probabilidade de do variável aleatória discreta $B_{u}$ ser igual a $h$ em uma raiz boa é definida por

$$
\begin{aligned}
P\left(B_{u}=h\right)_{G} & =\left(\begin{array}{l}
u \\
h
\end{array}\right) P\left(\overline{r_{i}}\right)_{G}^{h} P\left(r_{i}\right)_{G}^{u-h} \\
& =\left(\begin{array}{l}
u \\
h
\end{array}\right)\left((1-\delta)^{2}\right)^{h}\left(2 \delta(1-\delta)+\delta^{2}\right)^{u-h} .
\end{aligned}
$$

Com essas probabilidades já definidas podemos continuar com o cálculo de $\mathbb{E}[G]$ definido em (3.19), portanto temos

$$
\begin{array}{rlr}
\mathbb{E}[G] & =\sum_{h=1}^{u}\left(2^{h-1}-1\right) P\left(B_{u}=h\right)_{G}(P(d)+P(\bar{d})) & \\
& =\sum_{h=1}^{u}\left(2^{h-1}-1\right) P\left(B_{u}=h\right)_{G}(\delta+1-\delta) & \text { Substituindo } P(d) \text { e } P(\bar{d}) \\
& =\sum_{h=1}^{u}\left(2^{h-1}-1\right)\left(\begin{array}{l}
u \\
h
\end{array}\right)\left((1-\delta)^{2}\right)^{h}\left(2 \delta(1-\delta)+\delta^{2}\right)^{u-h}(\delta+1-\delta) & \text { Substituindo } P\left(B_{u}=h\right)_{G} \\
& =\sum_{h=1}^{u}\left(2^{h-1}-1\right)\left(\begin{array}{l}
u \\
h
\end{array}\right)(1-\delta)^{2 h}\left(2 \delta(1-\delta)+\delta^{2}\right)^{u-h} .
\end{array}
$$

Para entender a distribuição de probabilidade (veja a definição no Apêndice C.3) da variável aleatória discreta $G$ temos que calcular a sua variância e para isso devemos determinar o valor esperado de $G$ no momento de ordem 2 (veja a definição no Apêndice C.3.4). O valor esperado de $G$ no momento de ordem 2 é dado por $\mathbb{E}[G]$ já que em cada conjunto root $[j-1]$ para $1 \leq j \leq \frac{n}{u}$ existe só uma única raiz, portanto o valor esperado em qualquer momento é o mesmo (ver Figura $3.2)$ portanto temos que o valor de $\mathbb{E}\left[G^{2}\right]$ é dado por

$$
\mathbb{E}\left[G^{2}\right]=\mathbb{E}[G]=\sum_{h=1}^{u}\left(2^{h-1}-1\right)\left(\begin{array}{l}
u \\
h
\end{array}\right)(1-\delta)^{2 h}\left(2 \delta(1-\delta)+\delta^{2}\right)^{u-h} .
$$


Momento de ordem 1

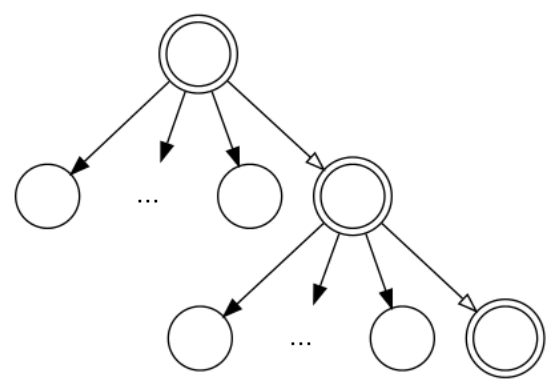

Figura 3.2: Representação dos momentos de ordem 1 e 2 da variável discreta $G$, onde podemos observar que ambos momentos são iguais.

\section{Valor Esperado do Número de Raízes Incorretas Geradas por uma Raiz Incorreta $(B)$}

Para determinar o valor esperado do número de raízes incorretas geradas por uma raiz incorreta $(\mathbb{E}[B])$, vamos definir uma raiz incorreta $r_{b}\left\langle r_{1}^{\prime}, r_{2}^{\prime}, d^{\prime}, d_{1}^{\prime}, d_{2}^{\prime},\left\langle r_{3}^{\prime}, d_{3}^{\prime}\right\rangle, \ldots,\left\langle r_{u}^{\prime}, d_{u}^{\prime}\right\rangle\right\rangle \in \operatorname{root}[j-1]$ onde seus valores calculados de

- $\left(N-\prod_{i=1}^{u} r_{i}^{\prime}\right)[j]$ pode ser pode ser igual a $c_{1}$ ou diferente (definido por $\overline{c_{1}}$ ).

- $\left(e d^{\prime}-1-k \prod_{i=1}^{u}\left(r_{i}^{\prime}-1\right)\right)[j+\tau(k)]$ pode ser igual a $c_{2}$ ou diferente (definido por $\overline{c_{2}}$ ).

- $\left(e d_{i}^{\prime}-1-k_{i}\left(r_{i}^{\prime}-1\right)\right)\left[j+\tau\left(k_{i}\right)\right]$ pode ser igual a $c_{3_{i}}$ ou diferente (definido por $\overline{c_{3_{i}}}$ ) para $1 \leq i \leq u$.

Tudo isso acontece já que existe muitas raízes incorretas no conjunto $\operatorname{root}[j-1]$ e cada um deles tem seus próprios valores calculados. Portanto em relação a uma raiz incorreta temos as seguintes relações nas equações $(3.16),(3.17)$ e (3.18)

$$
\begin{aligned}
& \left\{c_{1}, \overline{c_{1}}\right\} \equiv\left(N-\prod_{i=1}^{u} r_{i}^{\prime}\right)[j] \quad \equiv \sum_{i=1}^{u} r_{i}[j] \quad(\bmod 2) \\
& \left\{c_{2}, \overline{c_{2}}\right\} \equiv\left(e d^{\prime}-1-k \prod_{i=1}^{u}\left(r_{i}^{\prime}-1\right)\right)[j+\tau(k)] \equiv d[j+\tau(k)] \quad(\bmod 2) \\
& \left\{c_{3_{i}}, \overline{c_{3}}\right\} \equiv\left(e d_{i}^{\prime}-1-k\left(r_{i}^{\prime}-1\right)\right)\left[j+\tau\left(k_{i}\right)\right] \quad \equiv r_{i}[j]+d_{i}\left[j+\tau\left(k_{i}\right)\right] \quad(\bmod 2) \text { para } 1 \leq i \leq u .
\end{aligned}
$$

Devemos analisar todos os possíveis eventos que podem acontecer com relação aos valores calculados em uma raiz incorreta. Onde temos um total de $2^{u+2}$ cenários diferentes devido ao fato que cada valor calculado por ter dois valores $\left(\left\{c_{1}, \overline{c_{1}}\right\},\left\{c_{2}, \overline{c_{2}}\right\}\right.$ ou $\left\{c_{3_{i}}, \overline{c_{3_{i}}}\right\}$ para $\left.1 \leq i \leq u\right)$ e para cada cenário devemos analisar todos os eventos possíveis onde alguns bits de

$$
\left\langle r_{1}[j], r_{2}[j], d[j+\tau(k)], d_{1}\left[j+\tau\left(k_{1}\right)\right], d_{2}\left[j+\tau\left(k_{2}\right)\right],\left\langle r_{3}[j], d_{3}\left[j+\tau\left(k_{3}\right)\right]\right\rangle, \ldots,\left\langle r_{u}[j], d_{u}\left[j+\tau\left(k_{u}\right)\right]\right\rangle\right\rangle
$$

são conhecidos. E todo este cálculo deve ser feito para um valor $u$ que não é finito $(u \geq 2)$, o qual é uma tarefa bastante tediosa. O jeito de facilitar os cálculos é o que foi feito antes, já que existe uma Equação (3.18) para cada primo então pode ser usado para determinar uma nova probabilidade e com as equações (3.16) e (3.17) podemos calcular o número de raízes que são geradas, facilitando assim nosso cálculo na análise do valor esperado do número de raízes incorretas geradas por uma raiz incorreta.

\section{Número de raízes Incorretas geradas por uma raiz Incorreta}

Para o cálculo do número de raízes Incorretas geradas por uma raiz Incorreta só temos que analisar as equações 3.16 e 3.17 . Temos uma raiz incorreta $r_{b} \in \operatorname{root}[j-1]$ onde seus valores calculados para $\left(\left(N-\prod_{i=1}^{u} r_{i}^{\prime}\right)[j],\left(e d^{\prime}-1-k \prod_{i=1}^{u}\left(r_{i}^{\prime}-1\right)\right)[j+\tau(k)]\right)$ podem ser igual a um elemento do conjunto $\left\{\left(c_{1}, c_{2}\right),\left(c_{1}, \overline{c_{2}}\right),\left(\overline{c_{1}}, c_{2}\right),\left(\overline{c_{1}}, \overline{c_{2}}\right)\right\}$. Já definidos todos os eventos podem acontecer em uma raiz incorreta com relação a seus valores calculados, procedemos a analisar esses eventos com 


\begin{tabular}{|c|c||c|c|c|c|}
\hline$B_{u}$ & $d[j+\tau(k)]$ & $\left(c_{1}, c_{2}\right)$ & $\left(c_{1}, \overline{c_{2}}\right)$ & $\left(\overline{c_{1}}, c_{2}\right)$ & $\left(\overline{c_{1}}, \overline{c_{2}}\right)$ \\
\hline \hline $1 \leq h \leq u$ & unknown & $2^{h-1}$ & $2^{h-1}$ & $2^{h-1}$ & $2^{h-1}$ \\
$1 \leq h \leq u$ & known & $2^{h-1}$ & 0 & $2^{h-1}$ & 0 \\
$h=0$ & unknown & 1 & 1 & 0 & 0 \\
$h=0$ & known & 1 & 0 & 0 & 0 \\
\hline
\end{tabular}

Tabela 3.4: Número de raizes incorretas geradas a partir de uma raiz incorreta.

relação à fração $\delta$ de bits corretos que temos em $\tilde{s k}$ determinando o número de raízes incorretas que são geradas e que é mostrado na Tabela 3.4.

Sabemos que a variável aleatória discreta $B_{u}$ pode tomar o valor de $h$, onde $h$ é o numero de bits desconhecidos no grupo de bits $\left\langle r_{1}[j], r_{2}[j], \ldots, r_{u}[j]\right\rangle$ depois de ter sido analisado a Equação (3.18) de Hensel para $1 \leq i \leq u$ em uma raiz incorreta. Com o valor $h$ definido, procedemos a explicar todos os eventos que podem acontecer: O evento da primeira fila $1 \leq h \leq u$ com o bit $d[j+\tau(k)]$ desconhecido, temos que o número de raízes geradas está dado pela primeira Equação (3.16), onde temos uma equação com $h$ variáveis dando um total de $2^{h-1}$ raízes. E o valor para o bit $d[j+\tau(k)]$ pode ser calculado na Equação (3.16), e ele não altera o número de raízes geradas, portanto nos cenários $\left(\overline{c_{1}}, c_{2}\right),\left(c_{1}, \overline{c_{2}}\right),\left(\overline{c_{1}}, \overline{c_{2}}\right)$ e $\left(\overline{c_{1}}, \overline{c_{2}}\right)$ temos sempre um total de $2^{h-1}$ raízes geradas; No evento da segunda fila, $1 \leq h \leq u$ com o bit $d[j+\tau(k)]$ conhecido, temos que o número de raízes geradas é igual $2^{h-1}$ com relação à primeira Equação (3.16), mas como temos que o bit $d[j+\tau(k)]$ é conhecido então deve existir uma equivalência na Equação (3.17) para existir ditas raízes e isso só acontece quando temos os cenários $\left(c_{1}, c_{2}\right)$ e $\left(\overline{c_{1}}, c_{2}\right)$. Em os outros cenários $\left(c_{1}, \overline{c_{2}}\right)$ e $\left(\overline{c_{1}}, \overline{c_{2}}\right)$ as raízes geradas pela Equação (3.16) são eliminadas já que temos uma contradição na Equação (3.17); No penúltimo evento: $h=0$ com o bit $d[j+\tau(k)]$ desconhecido, temos uma única raiz gerada sempre e quando exista uma equivalência na Equação (3.16) e isso só existe nos cenários $\left(c_{1}, c_{2}\right)$ e $\left(c_{1}, \overline{c_{2}}\right)$, além disso o valor de $d[j+\tau(k)]$ pode ser calculado na Equação (3.17). Em os outros cenários $\left(\overline{c_{1}}, c_{2}\right)$ e $\left(\overline{c_{1}}, \overline{c_{2}}\right)$ não existe raiz gerada já que temos uma contradição na Equação (3.16); No último evento: $h=0$ com o bit $d[j+\tau(k)]$ conhecido, temos que todos os bits são conhecidos portanto no cenário $\left(c_{1}, c_{2}\right)$ obtemos duas equivalências com relação às equações (3.16) e (3.17) assim que temos uma única raiz gerada. Nos outros cenários $\left(\overline{c_{1}}, c_{2}\right),\left(c_{1}, \overline{c_{2}}\right)$ e $\left(\overline{c_{1}}, \overline{c_{2}}\right)$ sempre temos uma contradição em alguma das equações (3.16) ou (3.17), portanto não existe raiz gerada ou se ela existir é eliminada. Vale frisar que no cenário $\left(c_{1}, c_{2}\right)$ onde temos que os valores calculados da raiz incorreta são os mesmos da raiz boa, temos que o número de raízes geradas por uma raiz boa é igual ao número de raízes incorretas geradas pela raiz incorreta (veja a Tabela 3.1).

Com os resultados da Tabela 3.4 procedemos a determinar o valor esperado de $B$ obtendo que

$$
\begin{aligned}
\mathbb{E}[B]= & \sum_{i=1}^{u} 2^{h-1} P\left(B_{u}=h\right)_{B} P(\bar{d})\left[P\left(c_{1}\right) P\left(c_{2}\right)+P\left(c_{1}\right) P\left(\overline{c_{2}}\right)+P\left(\overline{c_{1}}\right) P\left(c_{2}\right)+P\left(\overline{c_{1}}\right) P\left(\overline{c_{2}}\right)\right]+ \\
& \sum_{i=1}^{u} 2^{h-1} P\left(B_{u}=h\right)_{B} P(d)\left[P\left(c_{1}\right) P\left(c_{2}\right)+P\left(\overline{c_{1}}\right) P\left(\overline{c_{2}}\right)\right]+ \\
& 1 P\left(B_{u}=0\right)_{B} P(\bar{d})\left[P\left(c_{1}\right) P\left(c_{2}\right)+P\left(c_{1}\right) P\left(\overline{c_{2}}\right)\right]+1 P\left(B_{u}=0\right)_{B} P(d) P\left(c_{1}\right) P\left(c_{2}\right)
\end{aligned}
$$

onde $P\left(B_{u}=h\right)_{B}$ é a probabilidade da variável aleatória discreta $B_{u}$ de ter o valor $h$ em uma raiz incorreta, $P\left(c_{1}\right), P\left(\overline{c_{1}}\right), P\left(c_{2}\right)$ e $P\left(\overline{c_{2}}\right)$ são as probabilidades definidas a seguir respectivamente $P\left(\left(N-\prod_{i=1}^{u} r_{i}^{\prime}\right)[j]=c_{1}\right), P\left(\left(N-\prod_{i=1}^{u} r_{i}^{\prime}\right)[j]=\overline{c_{1}}\right), P\left(\left(e d^{\prime}-1-k \prod_{i=1}^{u}\left(r_{i}^{\prime}-1\right)\right)[j+\tau(k)]=c_{2}\right) \mathrm{e}$ $P\left(\left(e d^{\prime}-1-k \prod_{i=1}^{u}\left(r_{i}^{\prime}-1\right)[j+\tau(k)]=\overline{c_{2}}\right)\right.$ e que serão calculadas a seguir. 


\section{Calculando as Probabilidades relacionadas a uma solução incorreta}

Com relação à Seção anterior temos as seguintes probabilidades

$$
\begin{aligned}
P\left(c_{1}\right) & =P\left(\left(N-\prod_{i=1}^{u} r_{i}^{\prime}\right)[j]=c_{1}\right) \\
P\left(c_{2}\right) & =P\left(\left(e d^{\prime}-1-k \prod_{i=1}^{u}\left(r_{i}^{\prime}-1\right)\right)[j+\tau(k)]=c_{2}\right) \\
P\left(c_{3_{i}}\right) & =P\left(\left(e d_{i}^{\prime}-1-k_{i}\left(r_{1}^{\prime}-1\right)\right)\left[j+\tau\left(k_{i}\right)\right]=c_{3_{i}}\right) \text { para } i \leq i \leq u .
\end{aligned}
$$

com seus respectivos complementos

$$
\begin{aligned}
P\left(\overline{c_{1}}\right) & =P\left(\left(N-\prod_{i=1}^{u} r_{i}^{\prime}\right)[j]=\overline{c_{1}}\right) \\
P\left(\overline{c_{2}}\right) & =P\left(\left(e d^{\prime}-1-k \prod_{i=1}^{u}\left(r_{i}^{\prime}-1\right)\right)[j+\tau(k)]=\overline{c_{2}}\right) \\
P\left(\overline{c_{3_{i}}}\right) & =P\left(\left(e d_{i}^{\prime}-1-k_{i}\left(r_{1}^{\prime}-1\right)\right)\left[j+\tau\left(k_{i}\right)\right]=\overline{c_{3_{i}}}\right) \text { para } i \leq i \leq u .
\end{aligned}
$$

As probabilidades definidas acima foram analisadas por Heninger [HS09], Kogure [KKY12] e Maitra [MSSG10]. Determinando que as probabilidades de cada valor calculado para uma raiz incorreta $r_{b}$ pode ter o valor de 0 ou 1 com uma probabilidade de $\frac{1}{2}$, o qual aplicado às probabilidades definidas temos que

$$
\begin{gathered}
P\left(c_{1}\right)=P\left(\overline{c_{1}}\right)=P\left(c_{2}\right)=P\left(\overline{c_{2}}\right)=\frac{1}{2} \quad \text { e } \\
P\left(c_{3_{i}}\right)=P\left(\overline{c_{3_{i}}}\right)=\frac{1}{2} \quad \text { para } 1 \leq i \leq u,
\end{gathered}
$$

As probabilidades $P\left(c_{3_{i}}\right)$ e $P\left(\overline{c_{3_{i}}}\right)$ para $1 \leq i \leq u$ são usadas para calcular a probabilidade de $P\left(B_{u}=h\right)_{B}$. Sabemos que o valor para $\left(e d_{i}^{\prime}-1-k_{i}\left(r_{i}^{\prime}-1\right)\right)\left[j+\tau\left(k_{i}\right)\right]$ pode ser igual a $c_{3_{i}}$ ou pode ser diferente (igual a $\overline{c_{3_{i}}}$ ), portanto vamos definir $P\left(r_{i}\right)_{B}$ e $P\left(\overline{r_{i}}\right)_{B}$, as quais são as probabilidades do valor do bit $r_{i}[j]$ ser conhecido ou desconhecido depois de ter analisado a Equação (3.18) para seu respectivo valor de $i$. Para determinar tais probabilidades temos analisado todos os eventos que podem acontecer com relação à Equação (3.18) na Tabela 3.5.

\begin{tabular}{|c|c||c|c|}
\hline \multicolumn{2}{|c|}{ Casos } & \multicolumn{2}{c|}{ Estado de $r_{i}[j]=\{$ known, unknown, $\nexists\}$} \\
\hline$r_{i}[j]$ & $d_{i}\left[j+\tau\left(k_{i}\right)\right]$ & $c_{3_{i}}$ & $\overline{c_{3_{i}}}$ \\
\hline \hline unknown & unknown & unknown & unknown \\
unknown & known & known & known \\
known & unknown & known & known \\
known & known & known & $\nexists$ \\
\hline
\end{tabular}

Tabela 3.5: Estado do bit $r_{i}[j]$ com relação ao estado do bit $d_{i}\left[j+\tau\left(k_{i}\right)\right]$ na raiz incorreta.

De acordo com a Tabela 3.5 temos os seguintes casos. Quando desconhecemos ambos bits $r_{i}[j]$ e $d_{i}\left[j+\tau\left(k_{i}\right)\right]$ o valor de $c_{3_{i}}$ ou $\overline{c_{3_{i}}}$ não é importante já que temos uma equação com duas variáveis a achar, portanto o bit $r_{i}[j]$ pode ter dois valores (0 ou 1) portanto ainda é desconhecido (unknown). $\mathrm{O}$ evento quando só um dos dois bits é conhecido com o valor de $c_{3_{i}}$ ou $\overline{c_{3_{i}}}$ temos uma equação com uma soa variável portanto o valor do bit $r_{i}[j]$ é conhecido (known) ou pode ser calculado. Por último, quando ambos bits $r_{i}[j]$ e $d_{i}\left[j+\tau\left(k_{i}\right)\right]$ são conhecidos devemos ter uma equivalência com $c_{3_{i}}$ para que $r_{i}[j]$ exista e seja conhecido, já que quando temos $\overline{c_{3_{i}}}$ acontece uma contradição portanto 
o valor de $r_{i}[j]$ é contraditório $(\nexists)$ e isso faz eliminar a raiz incorreta que está sendo analisada nesse instante.

Procedemos a determinar as probabilidades de $P\left(r_{i}\right)_{B}$ e $P\left(\bar{r}_{i}\right)_{B}$ de acordo com a Tabela 3.5 onde temos

$$
\begin{aligned}
P\left(r_{i}\right)_{B}=P\left(r_{i}[j] \leftarrow \text { known }\right)_{B}= & P\left(\overline{r_{i}}\right) P\left(d_{i}\right) P\left(c_{3_{i}}\right)+P\left(\overline{r_{i}}\right) P\left(d_{i}\right) P\left(\overline{c_{3_{i}}}\right)+P\left(r_{i}\right) P\left(\overline{d_{i}}\right) P\left(c_{3_{i}}\right)+ \\
& P\left(r_{i}\right) P\left(\overline{d_{i}}\right) P\left(\overline{c_{3_{i}}}\right)+P\left(r_{i}\right) P\left(d_{i}\right) P\left(c_{3_{i}}\right) \\
= & (1-\delta) \delta \frac{1}{2}+(1-\delta) \delta \frac{1}{2}+\delta(1-\delta) \frac{1}{2}+\delta(1-\delta) \frac{1}{2}+\delta \delta \frac{1}{2} \\
= & 2 \delta(1-\delta)+\frac{\delta^{2}}{2} \\
P\left(\overline{r_{i}}\right)_{B}=P\left(r_{i}[j] \leftarrow \text { unknown }\right)_{B}= & P\left(\overline{r_{i}}\right) P\left(\overline{d_{i}}\right) P\left(c_{3_{i}}\right)+P\left(\overline{r_{i}}\right) P\left(\overline{d_{i}}\right) P\left(\overline{c_{3_{i}}}\right) \\
= & (1-\delta)(1-\delta) \frac{1}{2}+(1-\delta)(1-\delta) \frac{1}{2} \\
= & (1-\delta)^{2} .
\end{aligned}
$$

Com essas probabilidades podemos definir que a probabilidade do evento $B_{u}$ ter $h$ bits desconhecidos em $\left\langle r_{1}[j], r_{2}[j], \ldots, r_{u}[j]\right\rangle$ em uma raiz incorreta é dada por

$$
\begin{aligned}
P\left(B_{u}=h\right)_{B} & =\left(\begin{array}{l}
u \\
h
\end{array}\right) P\left(\overline{r_{i}}\right)_{B}^{h} P\left(r_{i}\right)_{B}^{u-h} \\
& =\left(\begin{array}{l}
u \\
h
\end{array}\right)\left((1-\delta)^{2}\right)^{h}\left(2 \delta(1-\delta)+\frac{\delta^{2}}{2}\right)^{u-h} .
\end{aligned}
$$

Já analisado a probabilidades, voltamos a calcular $\mathbb{E}[B]$ que está definido em (3.23) onde temos que

$$
\begin{aligned}
\mathbb{E}[B]= & \sum_{i=1}^{u} 2^{h-1} P\left(B_{u}=h\right)_{B} P(\bar{d})\left[P\left(c_{1}\right) P\left(c_{2}\right)+P\left(c_{1}\right) P\left(\overline{c_{2}}\right)+P\left(\overline{c_{1}}\right) P\left(c_{2}\right)+P\left(\overline{c_{1}}\right) P\left(\overline{c_{2}}\right)\right]+ \\
& \sum_{i=1}^{u} 2^{h-1} P\left(B_{u}=h\right)_{B} P(d)\left[P\left(c_{1}\right) P\left(c_{2}\right)+P\left(\overline{c_{1}}\right) P\left(c_{2}\right)\right]+ \\
& 1 P\left(B_{u}=0\right)_{B} P(\bar{d})\left[P\left(c_{1}\right) P\left(c_{2}\right)+P\left(c_{1}\right) P\left(\overline{c_{2}}\right)\right]+1 P\left(B_{u}=0\right)_{B} P(d) P\left(c_{1}\right) P\left(c_{2}\right) .
\end{aligned}
$$


Onde substituindo as probabilidades $P\left(c_{1}\right), P\left(\overline{c_{1}}\right), P\left(c_{2}\right)$ e $P\left(\overline{c_{2}}\right)$ temos que

$$
\begin{aligned}
& \mathbb{E}[B]=\sum_{i=1}^{u} 2^{h-1} P\left(B_{u}=h\right)_{B} P(\bar{d})+\sum_{i=1}^{u} 2^{h-1} P\left(B_{u}=h\right)_{B} P(d) \frac{1}{2}+ \\
& 1 P\left(B_{u}=0\right)_{B} P(\bar{d}) \frac{1}{2}+1 P\left(B_{u}=0\right)_{B} P(d) \frac{1}{4} \\
& =\sum_{i=1}^{u} 2^{h-1} P\left(B_{u}=h\right)_{B} P(\bar{d})+\sum_{i=1}^{u} 2^{h-1} P\left(B_{u}=h\right)_{B} P(d) \frac{1}{2}+ \\
& 2^{0-1} P\left(B_{u}=0\right)_{B} P(\bar{d})+2^{0-1} P\left(B_{u}=0\right) P(d) \frac{1}{2} \\
& =\sum_{i=0}^{u} 2^{h-1} P\left(B_{u}=h\right)_{B} P(\bar{d})+\sum_{i=0}^{u} 2^{h-1} P\left(B_{u}=h\right)_{B} P(d) \frac{1}{2} \\
& =\left[\sum_{i=0}^{u} 2^{h-1} P\left(B_{u}=h\right)_{B}\right]\left[1-\delta+\frac{\delta}{2}\right] \\
& =\left[\sum_{i=0}^{u} 2^{h} P\left(B_{u}=h\right)_{B}\right] \frac{1}{2}\left[\frac{2-\delta}{2}\right] \\
& =\left[\sum_{i=0}^{u} 2^{h}\left(\begin{array}{l}
u \\
h
\end{array}\right)\left((1-\delta)^{2}\right)^{h}\left(2 \delta(1-\delta)+\frac{\delta^{2}}{2}\right)^{u-h}\right] \frac{1}{2}\left[\frac{2-\delta}{2}\right] \\
& =\left[\sum_{i=0}^{u}\left(\begin{array}{l}
u \\
h
\end{array}\right)\left(2(1-\delta)^{2}\right)^{h}\left(2 \delta(1-\delta)+\frac{\delta^{2}}{2}\right)^{u-h}\right] \frac{1}{2}\left[\frac{2-\delta}{2}\right] \\
& =\left(\left(2(1-\delta)^{2}+2 \delta(1-\delta)+\frac{\delta^{2}}{2}\right)^{u} \frac{1}{2}\left[\frac{2-\delta}{2}\right]\right. \\
& =\left(\frac{(2-2 \delta)^{2}+2 \delta(2-2 \delta)+\delta^{2}}{2}\right)^{u} \frac{1}{2}\left[\frac{2-\delta}{2}\right] \\
& =\left(\frac{(2-2 \delta+\delta)^{2}}{2}\right)^{u} \frac{1}{2}\left[\frac{2-\delta}{2}\right] \\
& =\frac{(2-\delta)^{2 u+1}}{2^{u+2}}
\end{aligned}
$$

Para entender a distribuição de probabilidade da variável discreta $B$ temos que calcular a variância e para isso devemos ter o valor esperado no momento de ordem 2 (veja no Apêndice C.8). Nesse casso podemos usar a fórmula para determinar já que uma solução incorreta só gera raízes incorretas e estas mais raízes incorretas (ver Figura 3.3) portanto $\mathbb{E}\left[B^{2}\right]$ é dado por

Momento de ordem 1

Momento de ordem 2

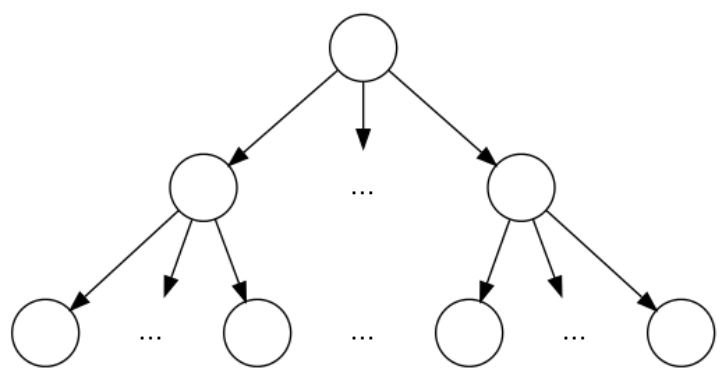

Figura 3.3: Representação dos momentos de ordem 1 e 2 da variável discreta $B$, onde podemos observar ambos momentos. 


$$
\begin{aligned}
\mathbb{E}\left[B^{2}\right]= & \sum_{i=1}^{u}\left(2^{h-1}\right)^{2} P\left(B_{u}=h\right)_{B} P(\bar{d})\left[P\left(c_{1}\right) P\left(c_{2}\right)+P\left(c_{1}\right) P\left(\overline{c_{2}}\right)+P\left(\overline{c_{1}}\right) P\left(c_{2}\right)+P\left(\overline{c_{1}}\right) P\left(\overline{c_{2}}\right)\right]+ \\
& \sum_{i=1}^{u}\left(2^{h-1}\right)^{2} P\left(B_{u}=h\right)_{B} P(d)\left[P\left(c_{1}\right) P\left(c_{2}\right)+P\left(\overline{c_{1}}\right) P\left(c_{2}\right)\right]+ \\
& 1^{2} P\left(B_{u}=0\right)_{B} P(\bar{d})\left[P\left(c_{1}\right) P\left(c_{2}\right)+P\left(c_{1}\right) P\left(\overline{c_{2}}\right)\right]+1 P^{2}\left(B_{u}=0\right)_{B} P(d) P\left(c_{1}\right) P\left(c_{2}\right),
\end{aligned}
$$

O qual pode ser expressado em função de $\mathbb{E}[B]$

$$
\begin{aligned}
\mathbb{E}\left[B^{2}\right]= & \sum_{i=1}^{u} 2^{h-1} P\left(B_{u}=h\right)_{B} P(\bar{d})\left[P\left(c_{1}\right) P\left(c_{2}\right)+P\left(c_{1}\right) P\left(\overline{c_{2}}\right)+P\left(\overline{c_{1}}\right) P\left(c_{2}\right)+P\left(\overline{c_{1}}\right) P\left(\overline{c_{2}}\right)\right]+ \\
& \sum_{i=1}^{u} 2^{h-1} P\left(B_{u}=h\right)_{B} P(d)\left[P\left(c_{1}\right) P\left(c_{2}\right)+P\left(\overline{c_{1}}\right) P\left(c_{2}\right)\right]+ \\
& 1 P\left(B_{u}=0\right)_{B} P(\bar{d})\left[P\left(c_{1}\right) P\left(c_{2}\right)+P\left(c_{1}\right) P\left(\overline{c_{2}}\right)\right]+1 P\left(B_{u}=0\right)_{B} P(d) P\left(c_{1}\right) P\left(c_{2}\right)+ \\
& \sum_{i=1}^{u}\left(2^{h-1}\right)\left(2^{h-1}-1\right) P\left(B_{u}=h\right)_{B} P(\bar{d})\left[P\left(c_{1}\right) P\left(c_{2}\right)+P\left(c_{1}\right) P\left(\overline{c_{2}}\right)+P\left(\overline{c_{1}}\right) P\left(c_{2}\right)+P\left(\overline{c_{1}}\right) P\left(\overline{c_{2}}\right)\right]+ \\
& \sum_{i=1}^{u}\left(2^{h-1}\right)\left(2^{h-1}-1\right) P\left(B_{u}=h\right)_{B} P(d)\left[P\left(c_{1}\right) P\left(c_{2}\right)+P\left(\overline{c_{1}}\right) P\left(c_{2}\right)\right]
\end{aligned}
$$

$\mathbb{E}\left[B^{2}\right]=\mathbb{E}[B]+$

$$
\begin{aligned}
& \sum_{i=1}^{u}\left(2^{h-1}\right)\left(2^{h-1}-1\right) P\left(B_{u}=h\right)_{B} P(\bar{d})\left[P\left(c_{1}\right) P\left(c_{2}\right)+P\left(c_{1}\right) P\left(\overline{c_{2}}\right)+P\left(\overline{c_{1}}\right) P\left(c_{2}\right)+P\left(\overline{c_{1}}\right) P\left(\overline{c_{2}}\right)\right]+ \\
& \sum_{i=1}^{u}\left(2^{h-1}\right)\left(2^{h-1}-1\right) P\left(B_{u}=h\right)_{B} P(d)\left[P\left(c_{1}\right) P\left(c_{2}\right)+P\left(\overline{c_{1}}\right) P\left(c_{2}\right)\right] .
\end{aligned}
$$

Onde substituindo as probabilidades $P\left(c_{1}\right), P\left(\overline{c_{1}}\right), P\left(c_{2}\right), P\left(\overline{c_{2}}\right), P(d)$ e $P(\bar{d})$ temos que

$$
\begin{aligned}
\mathbb{E}\left[B^{2}\right] & =\mathbb{E}[B]+\sum_{i=1}^{u}\left(2^{h-1}\right)\left(2^{h-1}-1\right) P\left(B_{u}=h\right)_{B}(1-\delta)+\sum_{i=1}^{u}\left(2^{h-1}\right)\left(2^{h-1}-1\right) P\left(B_{u}=h\right)_{B} \delta \frac{1}{2} \\
& =\mathbb{E}[B]+\sum_{i=1}^{u}\left(2^{h-1}\right)\left(2^{h-1}-1\right) P\left(B_{u}=h\right)_{B}\left[\frac{2-\delta}{2}\right]
\end{aligned}
$$

Fatorando

Por último substituímos as probabilidades $P\left(B_{u}=h\right)_{B}$ e o $\mathbb{E}[B]$ definido em (3.24).

$$
\mathbb{E}\left[B^{2}\right]=\frac{(2-\delta)^{2 u+1}}{2^{u+2}}+\sum_{i=1}^{u}\left(2^{h-1}\right)\left(2^{h-1}-1\right)\left(\begin{array}{l}
u \\
h
\end{array}\right)(1-\delta)^{2 h}\left(2 \delta(1-\delta)+\frac{\delta^{2}}{2}\right)^{u-h}\left[\frac{2-\delta}{2}\right]
$$

\section{Valor Esperado do Número de Raízes Incorretas no Nível $j$}

Para calcular o valor esperado do número de raízes incorretas no nível $j\left(\mathbb{E}\left[X_{j}\right]\right)$ temos que expressar tudo em relação de funções geradoras de probabilidades. Portanto uma FGP (veja a definição no Apêndice C.6.1) está definida pela função

$$
F(s)=\sum \operatorname{Pr}[X=k] s^{k}
$$


que representa a distribuição da variável aleatória discreta $X$ e que satisfaz as seguintes identidades:

$$
\begin{aligned}
F(1) & =1 \\
\mathbb{E}[X] & =F^{\prime}(1) \\
\operatorname{Var}[X] & =F^{\prime \prime}(1)+F^{\prime}(1)-F^{\prime}(1)^{2}
\end{aligned}
$$

Para isso vamos definir:

- Seja $F_{j}(s)$ a função geradora de probabilidades para v.a.d. $X_{j}$.

- Seja $g(s)$ a função geradora de probabilidades para v.a.d. $G$.

- Seja $b(s)$ a função geradora de probabilidades para v.a.d. $B$.

Onde temos que:

$$
\begin{aligned}
g^{\prime}(1) & =\mathbb{E}[G] & & \\
g^{\prime \prime}(1) & =\mathbb{E}\left[G^{2}\right]-\mathbb{E}[G] & & \text { Pela definição em C.14 } \\
b^{\prime}(1) & =\mathbb{E}[B] & & \\
b^{\prime \prime}(1) & =\mathbb{E}\left[B^{2}\right]-\mathbb{E}[B] & & \text { Pela definição em C.14 }
\end{aligned}
$$

\section{Calculando o Valor Esperado de $X_{j}$}

Por [HS09] e [KKY12] sabemos que a função $F_{j}$ determina a distribuição de probabilidade da v.a.d. $X_{j}$ e está definido por

$$
F_{j}(s)=F_{j-1}(b(s)) g(s),
$$

onde sua definição é porque o número de soluções incorretas geradas em cada passo é igual ao número de soluções incorretas geradas pelas soluções incorretas mais a soluções incorretas geradas pela solução correta. E além disso temos que $F_{0}(s)=1$ já que ao início não temos soluções incorretas.

Como queremos calcular o valor esperado da v.a.d $X_{j}$ então devemos calcular a derivada da função $F_{j}(s)$

$$
\begin{aligned}
& F_{j}(s)=F_{j-1}(b(s)) g(s) \\
& F_{j}^{\prime}(s)=F_{j-1}^{\prime}(b(s)) b^{\prime}(s) g(s)+F_{j-1}(b(s)) g^{\prime}(s) .
\end{aligned}
$$

Sabemos que $s=F_{j}(1)=b(1)=g(1)=F_{j-1}(1)=1$ por definição de FGP onde obtemos uma função de recorrência

$$
F_{j}^{\prime}(1)=F_{j-1}^{\prime}(1) b^{\prime}(1)+g^{\prime}(1) .
$$

Portanto procedemos a calcular a função fechada para essa função de recorrência

$$
\begin{array}{ll}
F_{j}^{\prime}(1)=F_{j-1}^{\prime}(1) b^{\prime}(1)+g^{\prime}(1) & \\
F_{j}^{\prime}(1)=\left(F_{j-2}^{\prime}(1) b^{\prime}(1)+g^{\prime}(1)\right) b^{\prime}(1)+g^{\prime}(1) & \text { Visto que } F_{j-1}^{\prime}=F_{j-2}^{\prime}(1) b^{\prime}(1)+g^{\prime}(1) \\
F_{j}^{\prime}(1)=F_{j-2}^{\prime}(1) b^{\prime}(1)^{2}+g^{\prime}(1) b^{\prime}(1)+g^{\prime}(1) & \\
F_{j}^{\prime}(1)=\left(F_{j-3}^{\prime}(1) b^{\prime}(1)+g^{\prime}(1)\right)(1) b^{\prime}(1)^{2}+g^{\prime}(1) b^{\prime}(1)+g^{\prime}(1) & \text { Visto que } F_{j-2}^{\prime}=F_{j-3}^{\prime}(1) b^{\prime}(1)+g^{\prime}(1) \\
F_{j}^{\prime}(1)=F_{j-3}^{\prime}(1) b^{\prime}(1)^{3}+g^{\prime}(1) b^{\prime}(1)^{2}+g^{\prime}(1) b^{\prime}(1)+g^{\prime}(1) . &
\end{array}
$$

Ao final obtemos

$$
F_{j}^{\prime}(1)=F_{0}^{\prime}(1) b^{\prime}(1)^{j}+g^{\prime}(1) b^{\prime}(1)^{j-1}+g^{\prime}(1) b^{\prime}(1)^{j-2}+\ldots+g^{\prime}(1) b^{\prime}(1)+g^{\prime}(1)
$$


onde $F_{0}^{\prime}(1)=0$ já que $F_{0}(1)=1$, portanto temos que

$$
\begin{array}{rlr}
F_{j}^{\prime}(1) & =g^{\prime}(1) \sum_{z=0}^{j-1} b^{\prime}(1)^{z} & \\
F_{j}^{\prime}(1) & =g^{\prime}(1) \sum_{z=1}^{j-1} b^{\prime}(1)^{z}+g^{\prime}(1) & \\
F_{j}^{\prime}(1)+g^{\prime}(1) b^{\prime}(1)^{j} & =g^{\prime}(1) \sum_{z=1}^{j} b^{\prime}(1)^{z}+g^{\prime}(1) & \\
F_{j}^{\prime}(1)+g^{\prime}(1) b^{\prime}(1)^{j} & =b^{\prime}(1) g^{\prime}(1) \sum_{z=0}^{j-1} b^{\prime}(1)^{z}+g^{\prime}(1) & \text { Propriedade de } \sum \\
g^{\prime}(1) b^{\prime}(1)^{j}+F_{j}^{\prime}(1) & =b^{\prime}(1)\left[F_{i}^{\prime}(1)\right]+g^{\prime}(1) & \text { Somando } g^{\prime}(1) b^{\prime}(1)^{j} \\
F_{j}^{\prime}(1)\left(1-b^{\prime}(1)\right) & =g^{\prime}(1)\left(1-b^{\prime}(1)^{j}\right) & \text { Fatorando } b^{\prime}(1) \\
F_{j}^{\prime}(1) & =g^{\prime}(1) \frac{\left(1-b^{\prime}(1)^{j}\right)}{\left(1-b^{\prime}(1)\right)} & \text { Definição de } F_{j}^{\prime}(1)
\end{array}
$$

onde substituindo pelos seus valores definidos em (3.26) temos que

$$
\mathbb{E}\left[X_{j}\right]=\mathbb{E}[G] \frac{\left(1-\mathbb{E}[B]^{j}\right)}{(1-\mathbb{E}[B])} .
$$

\section{Calculando a Variância de $X_{j}$}

Já calculado $\mathbb{E}\left[X_{j}\right]$ procedemos a determinar a variância $\mathbb{V} a r\left[X_{j}\right]$, o qual está definido por $\operatorname{Var}\left[X_{j}\right]=F_{j}^{\prime \prime}(1)+F_{j}^{\prime}(1)-F_{j}^{\prime}(1)^{2}$, portanto devemos calcular $F_{j}^{\prime \prime}(1)$ onde $F_{j}^{\prime \prime}(s)$ é a derivada da Função (3.27).

$$
\begin{aligned}
F_{j}^{\prime \prime}(s)= & F_{j-1}^{\prime \prime}(b(s)) b^{\prime}(s)\left(b^{\prime}(s) g(s)\right)+F_{j-1}^{\prime}(b(s))\left[b^{\prime \prime}(s) g(s)+b^{\prime}(s) g^{\prime}(s)\right]+ \\
& F_{j-1}^{\prime}(b(s)) b^{\prime}(s) g^{\prime}(s)+F_{j-1}(b(s)) g^{\prime \prime}(s)
\end{aligned}
$$

Onde sabemos que $s=F_{i}(1)=b(1)=g(1)=F_{j-1}(1)=1$, portanto temos que:

$$
\begin{aligned}
F_{j}^{\prime \prime}(1) & =F_{j-1}^{\prime \prime}(1) b^{\prime}(1)\left(b^{\prime}(1)+F_{j-1}^{\prime}(1)\left[b^{\prime \prime}(1)+b^{\prime}(1) g^{\prime}(1)\right]+F_{j-1}^{\prime}(1) b^{\prime}(1) g^{\prime}(1)+F_{j-1}(1) g^{\prime \prime}(1)\right. \\
& =F_{j-1}^{\prime \prime}(1) b^{\prime}(1)^{2}+F_{j-1}^{\prime}(1) b^{\prime \prime}(1)+2 F_{j-1}^{\prime}(1) b^{\prime}(1) g^{\prime}(1)+g^{\prime \prime}(1) \\
& =F_{j-1}^{\prime \prime}(1) b^{\prime}(1)^{2}+F_{j-1}^{\prime}(1)\left[b^{\prime \prime}(1)+2 b^{\prime}(1) g^{\prime}(1)\right]+g^{\prime \prime}(1)
\end{aligned}
$$

Na equação acima podemos substituir o valor de $F_{j-1}^{\prime}(1)$ já que ele é conhecido (Veja sua definição em (3.28))

$$
F_{j}^{\prime \prime}(1)=F_{j-1}^{\prime \prime}(1) b^{\prime}(1)^{2}+g^{\prime}(1) \frac{1-b^{\prime}(1)^{j-1}}{1-b^{\prime}(1)}\left[b^{\prime \prime}(1)+2 b^{\prime}(1) g^{\prime}(1)\right]+g^{\prime \prime}(1) .
$$

Para facilitar o cálculo da função fechada de $F_{j}^{\prime \prime}(1)$ vamos fazer algumas substituições. Substituindo $b^{\prime}(1)^{2}=a_{1}, g^{\prime}(1) \frac{1}{1-b^{\prime}(1)}\left[b^{\prime \prime}(1)+2 b^{\prime}(1) g^{\prime}(1)\right]=a_{2}$ e $a_{2}+g^{\prime \prime}(1)=a_{3}$ em $F_{j}^{\prime \prime}(1)$ obtemos então:

$$
F_{j}^{\prime \prime}(1)=F_{j-1}^{\prime \prime}(1) a_{1}-b^{\prime}(1)^{j-1} a_{2}+a_{3} .
$$


Calculando a função fechada

$F_{j}^{\prime \prime}(1)=F_{j-1}^{\prime \prime}(1) a_{1}-b^{\prime}(1)^{j-1} a_{2}+a_{3}$

$F_{j}^{\prime \prime}(1)=\left(F_{j-2}^{\prime \prime}(1) a_{1}-b^{\prime}(1)^{j-2} a_{2}+a_{3}\right) a_{1}-b^{\prime}(1)^{j-1} a_{2}+a_{3}$

Definição de $F_{j-1}^{\prime \prime}(1)$

$F_{j}^{\prime \prime}(1)=F_{j-2}^{\prime \prime}(1) a_{1}^{2}-b^{\prime}(1)^{j-2} a_{2} a_{1}+a_{3} a_{1}-b^{\prime}(1)^{j-1} a_{2}+a_{3}$

Definição de $F_{j-2}^{\prime \prime}(1)$

$F_{j}^{\prime \prime}(1)=\left(F_{j-3}^{\prime \prime}(1) a_{1}-b^{\prime}(1)^{j-3} a_{2}+a_{3}\right) a_{1}^{2}-b^{\prime}(1)^{j-2} a_{2} a_{1}+a_{3} a_{1}-b^{\prime}(1)^{j-1} a_{2}+a_{3}$

$F_{j}^{\prime \prime}(1)=F_{j-3}^{\prime \prime}(1) a_{1}^{3}-b^{\prime}(1)^{j-3} a_{2} a_{1}^{2}+a_{3} a_{1}^{2}-b^{\prime}(1)^{j-2} a_{2} a_{1}+a_{3} a_{1}-b^{\prime}(1)^{j-1} a_{2}+a_{3}$.

Continuando com as substituições ao final vamos obter que

$$
\begin{aligned}
& F_{j}^{\prime \prime}(1)=F_{1}^{\prime \prime}(1) a_{1}^{j-1}-b^{\prime}(1)^{1} a_{2} a_{1}^{j-2}+a_{3} a_{1}^{j-2}-b^{\prime}(1)^{2} a_{2} a_{1}^{j-3}+a_{3} a_{1}^{j-3}+\ldots-b^{\prime}(1)^{j-1} a_{2}+a_{3} \\
& F_{j}^{\prime \prime}(1)=F_{1}^{\prime \prime}(1) a_{1}^{j-1}+S_{1}+S_{2}
\end{aligned}
$$

onde temos que $S_{1}=-a_{2} \sum_{z=1}^{j-1} b^{\prime}(1)^{z} a_{1}^{j-1-z}$ e $S_{2}=a_{3} \sum_{z=0}^{j-2} a_{1}^{z}$

Resolvendo $S_{1}$

$$
\begin{array}{rlrl}
S_{1} & =-a_{2} \sum_{z=1}^{j-1} b^{\prime}(1)^{z} a_{1}^{j-1-z} & & \\
S_{1} & =-a_{2} \sum_{z=1}^{j-2} b^{\prime}(1)^{z} a_{1}^{j-1-z}-a_{2} b^{\prime}(1)^{j-1} & & \text { Propriedade de } \sum \\
S_{1} & =\frac{a_{1}}{b^{\prime}(1)}\left[-a_{2} \sum_{z=2}^{j-1} b^{\prime}(1)^{z} a_{1}^{j-1-z}\right]-a_{2} b^{\prime}(1)^{j-1} & & \text { Fatorando } \frac{a_{1}}{b^{\prime}(1)} \\
S_{1} & =\frac{a_{1}}{b^{\prime}(1)}\left[S_{1}+a_{2} b^{\prime}(1)^{1} a_{1}^{j-2}\right]-a_{2} b^{\prime}(1)^{j-1} & & \text { Definição de } S_{1} \\
S_{1}\left[1-\frac{a_{1}}{b^{\prime}(1)}\right] & =a_{2}\left[a_{1}^{j-1}-b^{\prime}(1)^{j-1}\right] & & \text { Fatorando } S_{1} \text { e } a_{2} \\
S_{1} & =a_{2} b^{\prime}(1) \frac{a_{1}^{j-1}-b^{\prime}(1)^{j-1}}{b^{\prime}(1)-a_{1}} &
\end{array}
$$

e $S_{2}$

$$
\begin{array}{rlr}
S_{2} & =a_{3} \sum_{z=0}^{j-2} a_{1}^{z} & \\
S_{2} & =a_{3} \sum_{z=1}^{j-2} a_{1}^{z}+a_{3} & \\
S_{2} & =a_{1}\left[a_{3} \sum_{z=0}^{j-1} a_{1}^{z}\right]+a_{3} & \text { Propriedade de } \sum \\
S_{2} & =a_{1}\left[S_{2}-a_{3} a_{1}^{j-2}\right]+a_{3} & \\
S_{2}\left(1-a_{1}\right) & =a_{3}\left(1-a_{1}^{j-1}\right) & \text { Fatorando } a_{1} \\
S_{2} & =a_{3} \frac{1-a_{1}^{j-1}}{1-a_{1}} &
\end{array}
$$


Substituindo $S_{1}$ e $S_{2}$ em $F_{j}^{\prime \prime}(1)$

$$
F_{j}^{\prime \prime}(1)=F_{1}^{\prime \prime}(1) a_{1}^{j-1}+a_{2} b^{\prime}(1) \frac{a_{1}^{j-1}-b^{\prime}(1)^{j-1}}{b^{\prime}(1)-a_{1}}+a_{3} \frac{1-a_{1}^{j-1}}{1-a_{1}}
$$

onde $F_{1}^{\prime \prime}(1)=g^{\prime \prime}(1)$ em $F_{1}^{\prime \prime}(1)=F_{0}^{\prime \prime}(1) b^{\prime}(1)^{2}+F_{0}^{\prime}(1) b^{\prime \prime}(1) g(1)+2 F_{0}^{\prime}(1) b^{\prime}(1) g^{\prime}(1)+g^{\prime \prime}(1)$ (ver sua definição em (3.30)) já que temos que $F_{0}^{\prime}(s)=F_{0}^{\prime \prime}(s)=0$ por ter $F_{o}(s)=1$, portanto

$$
F_{j}^{\prime \prime}(1)=g^{\prime \prime}(1) a_{1}^{j-1}+a_{2} b^{\prime}(1) \frac{a_{1}^{j-1}-b^{\prime}(1)^{j-1}}{b^{\prime}(1)-a_{1}}+a_{3} \frac{1-a_{1}^{j-1}}{1-a_{1}} .
$$

Substituindo $a_{1}=b^{\prime}(1)^{2}$ na equação acima.

$$
\begin{aligned}
& F_{j}^{\prime \prime}(1)=g^{\prime \prime}(1) b^{\prime}(1)^{2(j-1)}+a_{2} b^{\prime}(1) \frac{b^{\prime}(1)^{2(j-1)}-b^{\prime}(1)^{j-1}}{b^{\prime}(1)-b^{\prime}(1)^{2}}+a_{3} \frac{1-b^{\prime}(1)^{2(j-1)}}{1-b^{\prime}(1)^{2}} \\
& F_{j}^{\prime \prime}(1)=b^{\prime}(1)^{2(j-1)}\left[g^{\prime \prime}(1)+\frac{a_{2} b^{\prime}(1)}{b^{\prime}(1)-b^{\prime}(1)^{2}}-\frac{a_{3}}{1-b^{\prime}(1)^{2}}\right]-b^{\prime}(1)^{j-1}\left[\frac{a_{2} b^{\prime}(1)}{b^{\prime}(1)-b^{\prime}(1)^{2}}\right]+\frac{a_{3}}{1-b^{\prime}(1)^{2}} \\
& F_{j}^{\prime \prime}(1)=b^{\prime}(1)^{2(j-1)}\left[g^{\prime \prime}(1)+\frac{a_{2}}{1-b^{\prime}(1)}-\frac{a_{3}}{1-b^{\prime}(1)^{2}}\right]-b^{\prime}(1)^{j-1}\left[\frac{a_{2}}{1-b^{\prime}(1)}\right]+\frac{a_{3}}{1-b^{\prime}(1)^{2}}
\end{aligned}
$$

Substituindo $a_{3}=a_{2}+g^{\prime \prime}(1)$ na equação acima.

$$
\begin{aligned}
& F_{j}^{\prime \prime}(1)=b^{\prime}(1)^{2(j-1)}\left[g^{\prime \prime}(1)+\frac{a_{2}}{1-b^{\prime}(1)}-\frac{a_{2}+g^{\prime \prime}(1)}{1-b^{\prime}(1)^{2}}\right]-b^{\prime}(1)^{j-1}\left[\frac{a_{2}}{1-b^{\prime}(1)}\right]+\frac{a_{2}+g^{\prime \prime}(1)}{1-b^{\prime}(1)^{2}} \\
& F_{j}^{\prime \prime}(1)=b^{\prime}(1)^{2(j-1)}\left[g^{\prime \prime}(1)+\frac{a_{2}\left(1+b^{\prime}(1)\right)}{1-b^{\prime}(1)^{2}}-\frac{a_{2}+g^{\prime \prime}(1)}{1-b^{\prime}(1)^{2}}\right]-b^{\prime}(1)^{j-1}\left[\frac{a_{2}}{1-b^{\prime}(1)}\right]+\frac{a_{2}+g^{\prime \prime}(1)}{1-b^{\prime}(1)^{2}} \\
& F_{j}^{\prime \prime}(1)=b^{\prime}(1)^{2(j-1)}\left[g^{\prime \prime}(1)+\frac{a_{2} b^{\prime}(1)}{1-b^{\prime}(1)^{2}}-\frac{g^{\prime \prime}(1)}{1-b^{\prime}(1)^{2}}\right]-b^{\prime}(1)^{j-1}\left[\frac{a_{2}}{1-b^{\prime}(1)}\right]+\frac{a_{2}+g^{\prime \prime}(1)}{1-b^{\prime}(1)^{2}}
\end{aligned}
$$

Substituindo $a_{2}=g^{\prime}(1) \frac{1}{1-b^{\prime}(1)}\left[b^{\prime \prime}(1)+2 b^{\prime}(1) g^{\prime}(1)\right]$ na equação acima.

$$
\begin{aligned}
F_{j}^{\prime \prime}(1)= & b^{\prime}(1)^{2(j-1)}\left[g^{\prime \prime}(1)+\frac{g^{\prime}(1)\left[b^{\prime \prime}(1)+2 b^{\prime}(1) g^{\prime}(1)\right] b^{\prime}(1)}{\left(1-b^{\prime}(1)\right)\left(1-b^{\prime}(1)^{2}\right)}-\frac{g^{\prime \prime}(1)}{1-b^{\prime}(1)^{2}}\right] \\
& -b^{\prime}(1)^{j-1}\left[\frac{g^{\prime}(1)\left[b^{\prime \prime}(1)+2 b^{\prime}(1) g^{\prime}(1)\right]}{\left(1-b^{\prime}(1)\right)^{2}}\right] \\
& +\frac{1}{1-b^{\prime}(1)^{2}}\left[\frac{g^{\prime}(1)\left[b^{\prime \prime}(1)+2 b^{\prime}(1) g^{\prime}(1)\right]}{1-b^{\prime}(1)}+g^{\prime \prime}(1)\right]
\end{aligned}
$$

Com o valor $F_{j}^{\prime \prime}(1)$ já calculado retomamos o calculo de Variância de $X_{j}$

$$
\begin{aligned}
\operatorname{Var}\left[X_{j}\right]= & F_{j}^{\prime \prime}(1)+F_{j}^{\prime}(1)-F_{j}^{\prime}(1)^{2} \\
\operatorname{Var}\left[X_{j}\right]= & b^{\prime}(1)^{2(j-1)}\left[g^{\prime \prime}(1)+\frac{g^{\prime}(1)\left[b^{\prime \prime}(1)+2 b^{\prime}(1) g^{\prime}(1)\right] b^{\prime}(1)}{\left(1-b^{\prime}(1)\right)\left(1-b^{\prime}(1)^{2}\right)}-\frac{g^{\prime \prime}(1)}{1-b^{\prime}(1)^{2}}\right] \\
& -b^{\prime}(1)^{j-1}\left[\frac{g^{\prime}(1)\left[b^{\prime \prime}(1)+2 b^{\prime}(1) g^{\prime}(1)\right]}{\left(1-b^{\prime}(1)\right)^{2}}\right]+\frac{1}{1-b^{\prime}(1)^{2}}\left[\frac{g^{\prime}(1)\left[b^{\prime \prime}(1)+2 b^{\prime}(1) g^{\prime}(1)\right]}{1-b^{\prime}(1)}+g^{\prime \prime}(1)\right] \\
& +g^{\prime}(1) \frac{1-b^{\prime}(1)^{j}}{1-b^{\prime}(1)}-\left[g^{\prime}(1) \frac{1-b^{\prime}(1)^{j}}{1-b^{\prime}(1)}\right]^{2} .
\end{aligned}
$$

Com os valores calculados $\mathbb{E}\left[X_{j}\right]$ e $\operatorname{Var}\left[X_{j}\right]$ podemos determinar o número de raízes analisadas durante a execução do algoritmo. 


\section{Raízes Incorretas Analisadas Durante a Execução do Algoritmo}

Agora que sabemos a distribuição de probabilidade da variável aleatória discreta $X_{j}$, vamos determinar a distribuição do probabilidade do total de raízes incorretas analisadas na execução do programa. Como sabemos o algoritmo reconstrói a chave secreta $s k$ no nível $\frac{n}{u}$. Portanto devemos determinar o valor esperado e variância da somatória das raízes incorretas analisadas desde o nível 1 até o nível $\frac{n}{u}$, portanto devemos calcular $\mathbb{E}\left[\sum_{j=1}^{\frac{n}{u}} X_{j}\right]$ e $\operatorname{Var}\left[\sum_{j=1}^{\frac{n}{u}} X_{j}\right]$.

Calculando o Valor Esperado de $\mathbb{E}\left[\sum_{j=1}^{\frac{n}{u}} X_{j}\right]$

$$
\begin{array}{lr}
\mathbb{E}\left[\sum_{j=1}^{\frac{n}{u}} X_{j}\right]=\sum_{j=1}^{\frac{n}{u}} \mathbb{E}\left[X_{j}\right] & \text { Propriedade C.4 de valor esperado } \\
\mathbb{E}\left[\sum_{j=1}^{\frac{n}{u}} X_{j}\right]=\sum_{j=1}^{\frac{n}{u}} \mathbb{E}[G] \frac{1-\mathbb{E}[B]^{j}}{1-\mathbb{E}[B]} & \text { Definição (3.29) de } \mathbb{E}\left[X_{j}\right] \\
\mathbb{E}\left[\sum_{j=1}^{\frac{n}{u}} X_{j}\right]=\sum_{j=1}^{\frac{n}{u}} \frac{\mathbb{E}[G]}{1-\mathbb{E}[B]}-\sum_{j=1}^{\frac{n}{u}} \frac{\mathbb{E}[G] \mathbb{E}[B]^{j}}{1-\mathbb{E}[B]} & \text { Propriedade de } \sum \\
\mathbb{E}\left[\sum_{j=1}^{\frac{n}{u}} X_{j}\right]=\frac{n}{u} \frac{\mathbb{E}[G]}{1-\mathbb{E}[B]}+\frac{\mathbb{E}[G]}{\mathbb{E}[B]-1} \sum_{j=1}^{\frac{n}{u}} \mathbb{E}[B]^{j} & \text { Propriedade de } \sum
\end{array}
$$

Propriedade de $\sum$

Fatorando $\mathbb{E}[B]$

Propriedade de $\sum$

Fatorando $\sum_{j=1}^{\frac{n}{u}} \mathbb{E}[B]^{j}$ e $\mathbb{E}[B]$

$$
\sum_{j=1}^{\frac{n}{u}} \mathbb{E}[B]^{j}=\frac{\mathbb{E}[B]\left(1-\mathbb{E}[B]^{\frac{n}{u}}\right)}{1-\mathbb{E}[B]}=\frac{\mathbb{E}[B]\left(\mathbb{E}[B]^{\frac{n}{u}}-1\right)}{\mathbb{E}[B]-1}
$$

Portanto o valor esperado para o número total de raízes incorretas analisadas na execução do algoritmo é dado por

$$
\mathbb{E}\left[\sum_{j=1}^{\frac{n}{u}} X_{j}\right]=\frac{n}{u} \frac{\mathbb{E}[G]}{1-\mathbb{E}[B]}+\frac{\mathbb{E}[G] \mathbb{E}[B]\left(\mathbb{E}[B]^{\frac{n}{u}}-1\right)}{(\mathbb{E}[B]-1)^{2}} \quad \text { Substituindo } \sum_{j=1}^{\frac{n}{u}} \mathbb{E}[B]^{j}
$$


Calculando a Variância de $\operatorname{Var}\left[\sum_{j=1}^{\frac{n}{u}} X_{j}\right]$

Procedemos a calcular a variância do número de raízes incorretas analisadas pelo algoritmo.

$$
\begin{aligned}
\mathbb{V a r}\left[\sum_{j=1}^{\frac{n}{u}} X_{j}\right] & =\mathbb{E}\left[\left(\sum_{j=1}^{\frac{n}{u}} X_{j}\right)^{2}\right]-\mathbb{E}\left[\sum_{j=1}^{\frac{n}{u}} X_{j}\right]^{2} \\
& =\mathbb{E}\left[\sum_{l=1}^{\frac{n}{u}} \sum_{j=1}^{\frac{n}{u}} X_{l} X_{j}\right]-\mathbb{E}\left[\sum_{l=1}^{\frac{n}{u}} X_{l}\right] \mathbb{E}\left[\sum_{j=1}^{\frac{n}{u}} X_{j}\right] \\
& =\sum_{l=1}^{\frac{n}{u}} \sum_{j=1}^{\frac{n}{u}} \mathbb{E}\left[X_{l} X_{j}\right]-\sum_{l=1}^{\frac{n}{u}} \sum_{j=1}^{\frac{n}{u}} \mathbb{E}\left[X_{l}\right]\left[X_{j}\right] \\
& =\sum_{l=1}^{\frac{n}{u}} \sum_{j=1}^{\frac{n}{u}}\left(\mathbb{E}\left[X_{l} X_{j}\right]-\mathbb{E}\left[X_{l}\right]\left[X_{j}\right]\right) \\
& =\sum_{l=1}^{\frac{n}{u}} \sum_{j=1}^{\frac{n}{u}} \operatorname{Cov}\left(X_{l}, X_{j}\right) \\
& \leq \sum_{l=1}^{\frac{n}{u}} \sum_{j=1}^{\frac{n}{u}} \sqrt{\mathbb{V a r}\left[X_{l}\right] \mathbb{V a r}\left[X_{j}\right]} \\
& \leq \sum_{l=1}^{\frac{n}{u}} \sum_{j=1}^{\frac{n}{u}} \sqrt{\max \left(\mathbb{V}\left[X_{1}\right], \ldots, \mathbb{V a r}\left[X_{\frac{n}{u}}\right]\right)^{2}} \\
& \leq\left(\frac{n}{u}\right)^{2} \max \left(\mathbb{V a r}\left[X_{1}\right], \ldots, \mathbb{V a r}\left[X_{\frac{n}{u}}\right]\right)
\end{aligned}
$$

Definição C.5 de Variância

Propriedade C.4 de valor esperado Propriedade de $\sum$

Definição C.9 de Covariância

Pelo Coeficiente de Pearson (C.10)

Observa-se que a Definição (3.32)de valor esperado e a Definição (3.33) da sua respectiva variância do número de raízes analisadas durante a execução do Algoritmo 2 são dados por fórmulas exponenciais em função a $n$. Mas essa exponencialidade pode ser limitada dependendo do valor da base $\mathbb{E}[B]$. Quando temos que o valor $\mathbb{E}[B]>1$ podemos observar que o valor esperado e a variância são totalmente exponenciais já que $\lim _{n \rightarrow \infty} \mathbb{E}[B]^{\frac{n}{u}}=\infty$, mas quando o valor de $\mathbb{E}[B]$ é menor que 1 temos que o valor esperado e a variância do número de raízes analisadas na execução do algoritmo está limitado por funções polinomiais em função de $\frac{n}{u}$, e tudo isso acontece porque $\lim _{n \rightarrow \infty} \mathbb{E}[B]^{\frac{n}{u}}=0<1$. Em outras palavras, em (3.32) temos que

$$
\mathbb{E}\left[\sum_{j=1}^{\frac{n}{u}} X_{j}\right]=\frac{n}{u} \frac{\mathbb{E}[G]}{1-\mathbb{E}[B]}+\frac{\mathbb{E}[G] \mathbb{E}[B]\left(\mathbb{E}[B]^{\frac{n}{u}}-1\right)}{(\mathbb{E}[B]-1)^{2}} \leq \frac{n}{u} \frac{\mathbb{E}[G]}{1-\mathbb{E}[B]}
$$

e em (3.33)

$$
\begin{aligned}
\operatorname{Var}\left[\sum_{j=1}^{\frac{n}{u}} X_{j}\right] & \leq\left(\frac{n}{u}\right)^{2} \max \left(\operatorname{Var}\left[X_{1}\right], \ldots, \operatorname{Var}\left[X_{\frac{n}{u}}\right]\right) \\
& \leq\left(\frac{n}{u}\right)^{2}\left(\frac{\mathbb{E}[G]\left[\mathbb{E}\left[B^{2}\right]-\mathbb{E}[B]+2 \mathbb{E}[B] \mathbb{E}[G]\right]}{\left(1-\mathbb{E}[B]^{2}\right)(1-\mathbb{E}[B])}+\frac{\mathbb{E}[G]}{1-\mathbb{E}[B]}\right)
\end{aligned}
$$

onde o valor de $\left(\frac{\mathbb{E}[G]\left[\mathbb{E}\left[B^{2}\right]-\mathbb{E}[B]+2 \mathbb{E}[B] \mathbb{E}[G]\right]}{\left(1-\mathbb{E}[B]^{2}\right)(1-\mathbb{E}[B])}+\frac{\mathbb{E}[G]}{1-\mathbb{E}[B]}\right)$ é maior que $\max \left(\mathbb{V} \operatorname{ar}\left[X_{1}\right], \ldots, \operatorname{Var}\left[X_{\frac{n}{u}}\right]\right)$ e foi calculado a partir da Definição (3.31).

Já calculados o valor esperado e a variância do número de raízes incorretas analisadas durante 
a execução do algoritmo, podemos usar a definição da desigualdade de Chebyshev (ver o Apêndice C.3.3) para calcular a probabilidade em que nosso algoritmo analise um determinado número de raízes incorretas. Segundo à desigualdade de Chebychev temos que

$$
P(|X-\mathbb{E}[X]| \geq c \sigma) \leq \frac{1}{c^{2}}
$$

onde $c$ é uma constante e $\sigma$ é a desvio padrão $(\sigma=\sqrt{\operatorname{Var}[X]})$. Aplicando a desigualdade de Chebyshev para calcular a complexidade do algoritmo de reconstrução obtemos a seguinte expressão

$$
P\left(\left|\sum_{j=1}^{\frac{n}{u}} X_{j}-\mathbb{E}\left[\sum_{j=1}^{\frac{n}{u}} X_{j}\right]\right| \geq c \sqrt{\operatorname{Var}\left[\sum_{j=1}^{\frac{n}{u}} X_{j}\right]}\right) \leq \frac{1}{c^{2}}
$$

O valor para a constante $c$ é dado por $n$ para assim obter uma probabilidade em função a $n$, portanto temos que:

$$
P\left(\left|\sum_{j=1}^{\frac{n}{u}} X_{j}-\mathbb{E}\left[\sum_{j=1}^{\frac{n}{u}} X_{j}\right]\right| \geq n \sqrt{\operatorname{Var}\left[\sum_{j=1}^{\frac{n}{u}} X_{j}\right]}\right) \leq \frac{1}{n^{2}}
$$

O qual especifica que a probabilidade de analisar mais de $\left.\mathbb{E}\left[\sum_{j=1}^{\frac{n}{u}} X_{j}\right]+n \sqrt{\operatorname{Var}\left[\sum_{j=1}^{\frac{n}{u}} X_{j}\right.}\right]$ raízes incorretas é menor que $\frac{1}{n^{2}}$ ou pelo seu complemento podemos dizer que a probabilidade de analisar menos de

$$
\mathbb{E}\left[\sum_{j=1}^{\frac{n}{u}} X_{j}\right]+n \sqrt{\operatorname{Var}\left[\sum_{j=1}^{\frac{n}{u}} X_{j}\right]} \leq \frac{n}{u} \frac{\mathbb{E}[G]}{1-\mathbb{E}[B]}+\frac{n^{2}}{u} \sqrt{\max \left(\operatorname{Var}\left[X_{1}\right], \ldots, \operatorname{Var}\left[X_{\frac{n}{u}}\right]\right)}
$$

raízes incorretas é maior que $1-\frac{1}{n^{2}}$. Portanto podemos afirmar que a complexidade do algoritmo é de ordem $O\left(n^{2}\right)$ com uma probabilidade maior que $1-\frac{1}{n^{2}}$. Em conclusão, podemos reconstruir a chave secreta $s k$ do criptossistema RSA $(u \geq 2)$ em tempo polinomial com uma probabilidade maior que $1-\frac{1}{n^{2}}$, se temos uma porcentagem $\delta$ de bits de $s k$ em $\tilde{s k}$ maior que $2-2^{\frac{u+2}{2 u+1}}$. O valor de $\delta$ foi limitado quando foi definido que $\mathbb{E}[B]<1$.

$$
\begin{aligned}
\mathbb{E}[B]=\frac{(2-\delta)^{2 u+1}}{2^{u+2}} & <1 \\
(2-\delta)^{2 u+1} & <2^{u+2} \\
2-\delta & <2^{\frac{u+2}{2 u+1}} \\
\delta & >2-2^{\frac{u+2}{2 u+1}}
\end{aligned}
$$

\subsubsection{Alguns Resultados}

Para o criptossistema RSA básico $(u=2)$ temos que os valores esperados são dados por:

$$
\begin{aligned}
\mathbb{E}[G] & =(1-\delta)^{4} & & \text { por }(3.21) \\
\mathbb{E}\left[G^{2}\right] & =(1-\delta)^{4} & & \text { por }(3.22) \\
\mathbb{E}[B] & =\frac{(2-\delta)^{5}}{2^{4}} & & \text { por }(3.24) \\
\mathbb{E}\left[B^{2}\right] & =\frac{(2-\delta)^{5}}{2^{4}}+(2-\delta)(1-\delta)^{4} & & \text { por }(3.25)
\end{aligned}
$$


onde o valor de $\delta$ está limitado por $\delta \geq 2-2^{\frac{4}{5}} \approx 0.2589$. Os resultados do análise mostram que para um $\delta=0.26$, o número de raízes analisadas é menor que

$$
\mathbb{E}\left[\sum_{j=1}^{\frac{n}{u}} X_{j}\right]+n \sqrt{\operatorname{Var}\left[\sum_{j=1}^{\frac{n}{u}} X_{j}\right]} \leq \frac{n}{u} \frac{\mathbb{E}[G]}{1-\mathbb{E}[B]}+\frac{n^{2}}{u} \sqrt{\operatorname{Var}_{\max }\left[X_{j}\right]} \approx 48 n+45 n^{2}
$$

com uma probabilidade maior que $1-\frac{1}{n^{2}}$. Os valores apresentados acima podem ser corroborados no trabalho desenvolvido por Heninger e Shacham em [HS09].

Para o criptossistema RSA 3-primos temos os seguintes valores esperados

$$
\begin{aligned}
\mathbb{E}[G] & =3(1-\delta)^{4}\left(2 \delta(1-\delta)+\delta^{2}\right)+3(1-\delta)^{6} & & \text { por }(3.21) \\
\mathbb{E}\left[G^{2}\right] & =3(1-\delta)^{4}\left(2 \delta(1-\delta)+\delta^{2}\right)+3(1-\delta)^{6} & & \text { por }(3.22) \\
\mathbb{E}[B] & =\frac{(2-\delta)^{7}}{2^{5}} & & \text { por }(3.24) \\
\mathbb{E}\left[B^{2}\right] & =\frac{(2-\delta)^{7}}{2^{5}}+3(2-\delta)(1-\delta)^{4}\left(2 \delta(1-\delta)+\delta^{2} / 2\right)+6(2-\delta)(1-\delta)^{6} & & \text { por }(3.25)
\end{aligned}
$$

onde temos que $\delta \geq 2-2^{\frac{5}{7}} \approx 0.3593$. Portanto calculando o número de raízes analisadas para $\delta=0.36$ temos que é menor $59 n+62 n^{2}$ com uma probabilidade maior que $1-\frac{1}{n^{2}}$.

Por último, para o criptossistema RSA 4-primos temos os valores esperados

$$
\begin{aligned}
\mathbb{E}[G] & =\sum_{h=1}^{4}\left(2^{h-1}-1\right)\left(\begin{array}{l}
4 \\
h
\end{array}\right)(1-\delta)^{2 h}\left(2 \delta(1-\delta)+\delta^{2}\right)^{4-h} \\
\mathbb{E}\left[G^{2}\right] & =\sum_{h=1}^{4}\left(2^{h-1}-1\right)\left(\begin{array}{l}
4 \\
h
\end{array}\right)(1-\delta)^{2 h}\left(2 \delta(1-\delta)+\delta^{2}\right)^{4-h} \\
\mathbb{E}[B] & =\frac{(2-\delta)^{9}}{2^{6}} \\
\mathbb{E}\left[B^{2}\right] & =\frac{(2-\delta)^{9}}{2^{6}}+\sum_{i=1}^{4}\left(2^{h-1}\right)\left(2^{h-1}-1\right)\left(\begin{array}{l}
4 \\
h
\end{array}\right)(1-\delta)^{2 h}\left(2 \delta(1-\delta)+\frac{\delta^{2}}{2}\right)^{4-h}\left[\frac{2-\delta}{2}\right]
\end{aligned}
$$

com o valor $\delta$ limitado $\delta \geq 2-2^{\frac{6}{9}} \approx 0.4126$. Portanto calculando o número de raízes analisadas para $\delta=0.42$ temos que é menor que $5 n+5 n^{2}$. 


\section{Capítulo 4}

\section{Implementação e Resultados}

\subsection{Implementação}

Para a implementação do Algoritmo 2 usamos linguagem de programação C e a biblioteca criptográfica Relic Toolkit [AG] pelas seguintes razões a seguir

1. Não existe limite de precisão do tamanho dos inteiros

2. Tem implementado aritmética para corpos binários e primos.

3. Máxima eficiência.

4. Configuração flexível

Além disso, o RELIC é uma das poucas bibliotecas focada à criptografia e é distribuída gratuitamente sob a GNU Lesser General Public License (veja [GNU] e [AG]).

O algoritmo de reconstrução foi implementado para a reconstrução de chaves secretas dos criptossistemas RSA básico, 3-primos e 4-primos. A implementação foi feita em menos de 500 linhas de código e os testes foram executados sobre o servidor (brucutu) das instalações do IME-USP ${ }^{1}$. O servidor tem as seguintes especificações: Um total de 22 processadores Intel(R) Xeon(R) $2.40 \mathrm{GHz}$, $12 \mathrm{MB}$ de cache e 126 GB de memória DDR3. Mas para nossos experimentos só foi usado um único processador.

Os experimentos realizados foram feitos para chaves de 2048, onde para cada valor de $u$ foi dado um intervalo de valores para $\delta$ :

- Para o criptossistema RSA básico o valores de $\delta$ estão entre [0.24, 0.45].

- Para o criptossistema RSA multi-primo $(u=3)$, o valores de $\delta$ estão entre $[0.34,0.45]$.

- Para o criptossistema RSA multi-primo $(u=4)$, o valores de $\delta$ estão entre $[0.40,0.45]$.

Todos eles têm como valor máximo de $\delta$, o valor de 0.45 para fins de comparação entre eles. Para cada valor de $\delta$ e criptossistema RSA foram geradas um total de 100 criptossistemas RSA aleatoriamente e para cada criptossistema RSA foi gerado um total de 100 chaves deterioradas com uma fração $\delta$ de bits corretos fazendo um total de 400000 experimentos para chaves de 2048 bits.

Em todos os criptossistemas RSA gerados o expoente de encriptação sempre foi o valor $e=$ 65537. Além disso para evitar dados desnecessários, os experimentos foram executados com os valores $\left\langle k, k_{1}, \ldots, k_{u}\right\rangle$ já calculados.

\footnotetext{
${ }^{1}$ Instituto de Matemáticas e Estatística - Universidade de São Paulo
} 


\subsection{Resultados para Chaves de 2048 Bits}

Os resultados dos experimentos do algoritmo de reconstrução da chave secreta do criptossistema RSA $(2 \leq u \leq 4)$ feitos para um módulo $N$ de 2048 bits, estão sintetizados nas tabelas 4.1, 4.2 e 4.3 e na Imagem 4.1. As tabelas estão organizadas do seguinte jeito: A primeira coluna especifica o valor da fração de bits corretos em $\tilde{s k}$, dos experimentos a realizar. A segunda e terceira colunas mostram o mínimo e o máximo número de raízes analisadas em todos os experimentos realizados. A quarta e a quinta colunas contêm o valor médio e a variância do número de raízes analisadas dos experimentos que tiveram sucesso em relação à reconstrução da chave secreta A quinta coluna mostra quantos experimentos foram cancelados por ultrapassar um milhão de raízes analisadas. Onde um milhão de raízes analisadas é uma quantidade bastante grande, e segundo a desigualdade de Chebyshev isso acontece com uma probabilidade muito baixa. Por último, na sétima coluna temos o tempo médio que o Algoritmo 2 demorou na reconstrução das chaves secretas.

\subsubsection{Resultados dos Experimentos do Criptossistema RSA Básico}

\begin{tabular}{|c||c|c|c|c|r|c|}
\hline \multicolumn{1}{|c||}{} & \multicolumn{2}{c|}{ Quantidade de soluções analisadas } & \# exp. & Tempo \\
\hline$\delta$ & Mínimo & Máximo & Média & Variância & $(>1 \mathrm{M})$ & Média (s) \\
\hline \hline 0.45 & 1127 & 1638 & 1228 & $3.569 \times 10^{3}$ & 0 & 0.019169 \\
0.44 & 1135 & 1720 & 1264 & $4.594 \times 10^{3}$ & 0 & 0.019984 \\
0.43 & 1145 & 1767 & 1294 & $4.847 \times 10^{3}$ & 0 & 0.020577 \\
0.42 & 1182 & 2016 & 1330 & $6.083 \times 10^{3}$ & 0 & 0.021478 \\
0.41 & 1195 & 1959 & 1362 & $8.290 \times 10^{3}$ & 0 & 0.022426 \\
0.40 & 1202 & 2170 & 1399 & $1.202 \times 10^{4}$ & 0 & 0.023212 \\
0.39 & 1224 & 4310 & 1453 & $1.078 \times 10^{4}$ & 0 & 0.024319 \\
0.38 & 1273 & 3583 & 1531 & $1.437 \times 10^{4}$ & 0 & 0.025600 \\
0.37 & 1309 & 5803 & 1616 & $2.461 \times 10^{4}$ & 0 & 0.028092 \\
0.36 & 1354 & 15867 & 1676 & $3.063 \times 10^{4}$ & 0 & 0.029307 \\
0.35 & 1385 & 5915 & 1797 & $5.723 \times 10^{4}$ & 0 & 0.031717 \\
0.34 & 1438 & 5444 & 1954 & $9.889 \times 10^{4}$ & 0 & 0.035030 \\
0.33 & 1519 & 19939 & 2152 & $1.291 \times 10^{5}$ & 0 & 0.037946 \\
0.32 & 1581 & 26863 & 2406 & $3.374 \times 10^{5}$ & 0 & 0.044103 \\
0.31 & 1693 & 21654 & 2606 & $7.748 \times 10^{5}$ & 0 & 0.050284 \\
0.30 & 1747 & 123021 & 3191 & $5.463 \times 10^{6}$ & 0 & 0.058877 \\
0.29 & 2036 & 571178 & 4012 & $1.098 \times 10^{7}$ & 0 & 0.078551 \\
0.28 & 2283 & 776810 & 5060 & $5.633 \times 10^{7}$ & 0 & 0.110014 \\
0.27 & 2555 & 850244 & 7788 & $2.589 \times 10^{8}$ & 3 & 0.149914 \\
\hline \hline 0.26 & 2994 & 977055 & 14624 & $8.257 \times 10^{8}$ & 10 & 0.271213 \\
0.25 & 4029 & 982756 & 26879 & $4.147 \times 10^{9}$ & 49 & 0.623392 \\
0.24 & 4939 & 996729 & 60232 & $1.108 \times 10^{10}$ & 245 & 1.299131 \\
\hline
\end{tabular}

Tabela 4.1: Resultados dos experimentos da Reconstrução da Chave Secreta RSA Básico $(u=2)$.

Os resultados dos experimentos com relação ao criptossistema RSA básico mostram que é possível a reconstrução das chaves secretas RSA de 2048 bits, com um tempo médio menor que 1 segundo para valores $\delta \geq 0.26$. Para valores $\delta \leq 0.26$, na Tabela 4.1 é visto um crescimento do tipo exponencial no tempo médio, a média e na variância do número de raízes analisadas. Além disso temos que o número de experimentos que são eliminados cresce quanto o valor de $\delta$ fica longe de 0.27 .

Segundo os resultados da Seção 3.4.2 sabemos que teoricamente vamos analisar uma quantidade de raízes incorretas menor que $48 n+45 n^{2}$ para um valor $\delta=0.26$, o qual é ainda uma quantidade 
bastante grande (os resultados dos experimentos corroboram esses dados). Mas para um $\delta=0.27 \mathrm{o}$ número de raízes analisadas diminui quadraticamente, seguindo a equação $5 n+5 n^{2}$, o qual é uma quantidade bastante aceitável com relação a 0.26. Por isso Heninger e Shacham no seu artigo [HS09] declaram que é preciso um $\delta \geq 0.27$ para reconstruir a chave secreta do criptossistema RSA básico em tempo polinomial.

Para a reconstrução da chave secreta no criptossistema RSA básico tivemos um total de 307 experimentos onde a reconstrução de $s k$ não foi possível de um total de 220000 de experimentos, tendo uma efetividade de $99.86 \%$ para valores de $0.24 \leq \delta \leq 0.45$. Para valores de $0.27 \leq \delta \leq 0.45$ só obtivemos 3 experimentos cancelados de um total de 190000 (99.99\% de efetividade).

\subsubsection{Resultados dos Experimentos do Criptossistema RSA 3-primos}

Com relação ao criptossistema RSA 3-primos os resultados dos experimentos estão descritos na Tabela 4.2. Onde de acordo com os resultados da Seção 3.4.2 sabemos que teoricamente o número de raízes incorretas analisadas é menor que $59 n+62 n^{2}$ para um valor $\delta=0.36$, o qual é uma quantidade bastante grande (os resultados dos experimentos corroboram esses dados). Mas para um $\delta=0.37$ o número de raízes analisadas diminui quadraticamente, seguindo a equação $4 n+4 n^{2}$, o qual é aceitável com relação a 0.37. Por isso vamos declarar que para reconstruir a chave secreta do criptossistema RSA 3-primos em tempo polinomial é preciso um $\delta \geq 0.37$.

Para a reconstrução da chave secreta no criptossistema RSA 3-primos foram feitos um total de 120000 experimentos, onde 200 foram cancelados porque ultrapassam um milhão de raízes incorretas analisadas, obtendo uma efetividade do $99.83 \%$ para valores de $0.34 \leq \delta \leq 0.45$. Para valores de $0.37 \leq \delta \leq 0.45$ só foram 2 experimentos cancelados de um total de 90000 (99.99\% de efetividade).

A reconstrução da chave secreta RSA 3-primos para $\delta \geq 0.37$ é feita com um tempo médio menor que 2 segundos.

\begin{tabular}{|c||c|c|c|c|r|c|}
\hline \multicolumn{1}{|c||}{} & \multicolumn{9}{c|}{ Quantidade de soluções analisadas } & \# exp. & Tempo \\
\hline$\delta$ & Mínimo & Máximo & Média & Variância & $(>1 \mathrm{M})$ & Média (s) \\
\hline \hline 0.45 & 1128 & 6595 & 1542 & $8.348 \times 10^{4}$ & 0 & 0.091892 \\
0.44 & 1201 & 13701 & 1700 & $2.380 \times 10^{5}$ & 0 & 0.103603 \\
0.43 & 1221 & 35902 & 1972 & $2.202 \times 10^{5}$ & 0 & 0.115990 \\
0.42 & 1420 & 16786 & 2295 & $6.925 \times 10^{5}$ & 0 & 0.147067 \\
0.41 & 1503 & 24375 & 2538 & $2.533 \times 10^{6}$ & 0 & 0.175805 \\
0.40 & 1625 & 500611 & 3406 & $1.351 \times 10^{7}$ & 0 & 0.218010 \\
0.39 & 1711 & 147153 & 4116 & $7.520 \times 10^{7}$ & 0 & 0.304466 \\
0.38 & 2140 & 388317 & 5638 & $1.385 \times 10^{8}$ & 1 & 0.412533 \\
0.37 & 2288 & 713089 & 8645 & $6.597 \times 10^{8}$ & 1 & 0.635832 \\
\hline \hline 0.36 & 2613 & 928901 & 1345 & $1.506 \times 10^{9}$ & 14 & 1.134340 \\
0.35 & 3878 & 964553 & 24107 & $3.201 \times 10^{9}$ & 28 & 1.868184 \\
0.34 & 4218 & 997646 & 53173 & $1.082 \times 10^{10}$ & 156 & 4.199117 \\
\hline
\end{tabular}

Tabela 4.2: Resultados dos experimentos da Reconstrução da Chave Secreta RSA 3-primos.

\subsubsection{Resultados dos Experimentos do Criptossistema RSA 4-primos}

Os resultados dos experimentos em relação ao criptossistema RSA 4-primos estão contidos na Tabela 4.3 onde a reconstrução da chave secreta $s k$ acontece em tempo médio menor que 5 segundos para valores de $\delta \geq 0.42$. Do total de 60000 experimentos, só 202 experimentos não tiveram sucesso na reconstrução da chave secreta já que foram cancelados ( $99.66 \%$ de efetividade). Para valores de $0.42 \leq \delta \leq 0.45$, a reconstrução da chave secreta teve uma efetivada de $99.99 \%$ (3 experimentos cancelados de um total de 40000 experimentos). 


\begin{tabular}{|c||c|c|c|c|r|c|}
\hline \multicolumn{1}{|c||}{} & \multicolumn{9}{c|}{ Quantidade de soluções analisadas } & \# exp. & Tempo \\
\hline$\delta$ & Mínimo & Máximo & Média & Variância & $(>1 \mathrm{M})$ & Média (s) \\
\hline \hline 0.45 & 1750 & 172975 & 4781 & $2.022 \times 10^{7}$ & 0 & 0.355657 \\
0.44 & 2251 & 642073 & 6714 & $1.838 \times 10^{8}$ & 0 & 0.572618 \\
0.43 & 2084 & 344425 & 9216 & $1.993 \times 10^{8}$ & 0 & 0.774536 \\
0.42 & 2882 & 776803 & 16484 & $1.524 \times 10^{8}$ & 3 & 1.497140 \\
\hline \hline 0.41 & 3802 & 981081 & 30423 & $4.425 \times 10^{9}$ & 72 & 2.640792 \\
0.40 & 5796 & 986662 & 63909 & $8.758 \times 10^{9}$ & 127 & 4.220306 \\
0.39 & 8052 & 999313 & 90386 & $1.763 \times 10^{10}$ & 310 & 7.316338 \\
\hline
\end{tabular}

Tabela 4.3: Resultados dos experimentos da Reconstrução da Chave Secreta RSA 4-primos.

\subsubsection{Comparações}

Os resultados dos experimentos feitos e explicados para chaves de 2048 bits corroboram experimentalmente nossa análise da complexidade do algoritmo de reconstrução da chave secreta RSA (ver Seção 3.4.2). Nas tabelas observamos que o tempo para reconstruir uma chave secreta sk é maior enquanto o valor de $u$ cresce. Isso acontece porque o tempo em gerar e analisar uma raiz é mais demorado, já que uma raiz gerada é trabalhada com relação a $2 u+1$ variáveis da chave secreta e devem ser verificados um total de $u+2$ equivalências (equações (3.16), (3.17) e (3.18) de Hensel). Além disso, nos experimentos para os criptossistemas RSA $(2 \leq u \leq 4)$ tendo um mesmo valor $\delta$, observamos que o valor médio do número de raízes analisadas cresce enquanto o valor de $u$ for maior, e isso acontece porque os valores esperados de $G$ e $B(\mathbb{E}[G]$ e $\mathbb{E}[B])$ também crescem. Portanto temos que para um RSA multi-primo vamos analisar uma quantidade maior de raízes onde cada uma delas vai demorar um tempo maior.

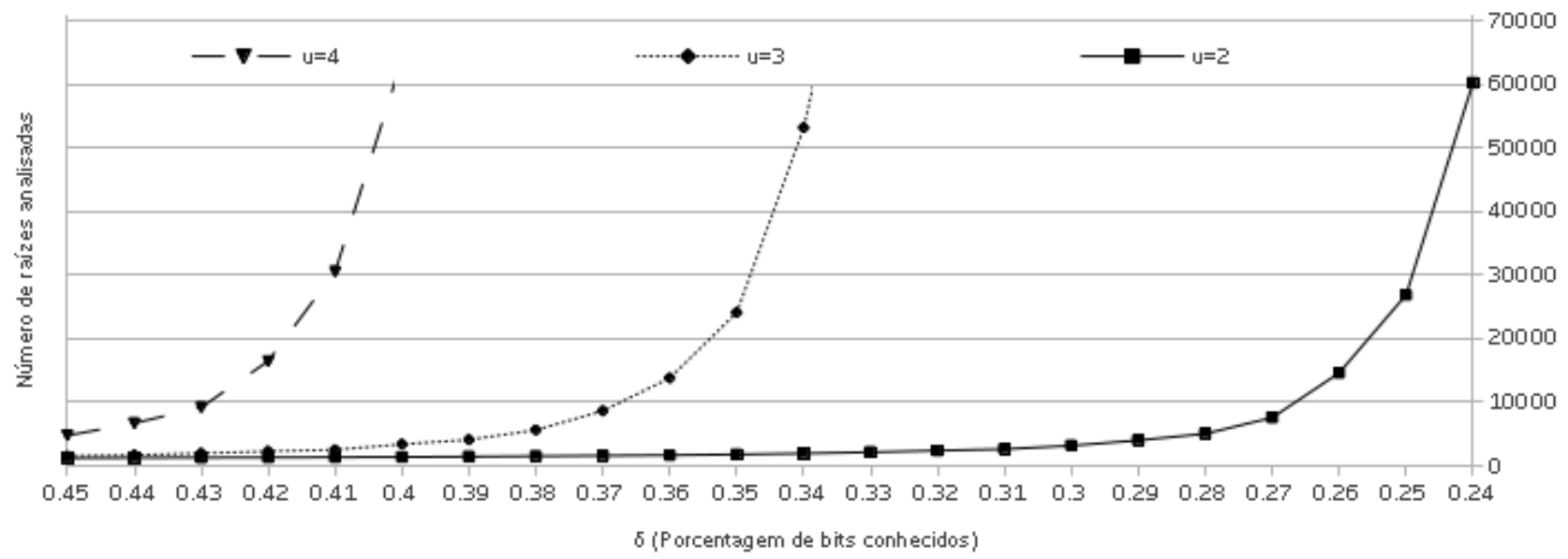

Figura 4.1: Comparação das médias da quantidade de raízes analisadas para chaves de 2048 bits.

Na Imagem 4.1 podemos observar a comparação entre a média de raízes analisadas das tabelas 4.1, 4.2 e 4.3 onde é fácil observar o comportamento do algoritmo com relação a cada criptossistema RSA, onde podemos concluir que é possível a reconstrução das chaves secretas do criptossistema:

- RSA básico tendo um valor $\delta \geq 0.27$

- RSA 3-primos tendo um valor $\delta \geq 0.37$

- RSA 4-primos tendo um valor $\delta \geq 0.42$

em tempo eficiente. Mostrando assim que a segurança oferecida pelo criptossistema: RSA 4-primos é maior com relação ao RSA 3-primos e básico, RSA 3-primos é maior com relação ao RSA básico. 


\subsection{Experimentos Extras}

O experimentos que foram analisados foram feitos para chaves secretas de 2048 bits para os criptossistemas RSA básico, 3-primos e 4-primos, Nesta seção serão mencionados os resultados dos experimentos com relação a chaves secretas de 3072 e 4096 bits nas tabelas 4.4 e 4.5 respectivamente. Os resultados obtidos não diferem com os obtidos anteriormente feitos para chaves de 2048 bits, portanto não necessita explicação auxiliar.

O número total de experimentos que foram executados está dado pelas especificações dos experimentos para chaves de 2048 bits. Foram realizados um total de 800000 experimentos extras (400000 experimentos com chaves de 3072 bits e 400000 experimentos com chaves de 4096 bits).

\subsubsection{Experimentos para chaves de 3072 bits}

Os resultados dos experimentos realizados para chaves de 3072 bits mostram:

- Um comportamento similar em relação aos valores de $\delta$

- Que a razão entre o número médio de raízes analisadas e o tempo médio de reconstrução das chaves apresenta uma proporção igual a $\frac{3072}{2048}=\frac{3}{2}$

com relação aos experimentos realizados para chaves de 2048 bits.

\begin{tabular}{|c|c|c|c|c|c|c|c|c|c|}
\hline & \multicolumn{3}{|c|}{ Criptossistema RSA básico } & \multicolumn{3}{|c|}{ Criptossistema RSA 3-primos } & \multicolumn{3}{|c|}{ Criptossistema RSA 4-primos } \\
\hline & \multicolumn{2}{|c|}{ \# exp. } & \multirow{2}{*}{$\begin{array}{c}\text { Tempo } \\
\text { Média (s) }\end{array}$} & \multicolumn{2}{|c|}{ \# exp. } & \multirow{2}{*}{$\begin{array}{c}\text { Tempo } \\
\text { Média (s) }\end{array}$} & \multicolumn{2}{|c|}{ " \# exp. } & \multirow{2}{*}{$\begin{array}{c}\text { Tempo } \\
\text { Média (s) }\end{array}$} \\
\hline$\delta$ & Média & $(>1 \mathrm{M})$ & & Média & $(>1 \mathrm{M})$ & & Média & $(>1 \mathrm{M})$ & \\
\hline 0.45 & 1850 & 0 & 0.045115 & 2446 & 0 & 0.239377 & 7512 & 0 & 0.992764 \\
\hline 0.44 & 1900 & 0 & 0.045115 & 2577 & 0 & 0.277263 & 9904 & 0 & 1.389699 \\
\hline 0.43 & 1948 & 0 & 0.048160 & 2950 & 0 & 0.317987 & 15273 & 4 & 2.227851 \\
\hline 0.42 & 1975 & 0 & 0.049974 & 3389 & 0 & 0.387352 & 19684 & 14 & 3.342511 \\
\hline 0.41 & 2047 & 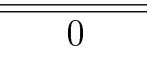 & 0.052617 & 3879 & 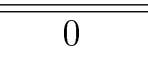 & 0.628048 & 38416 & 114 & 6.023370 \\
\hline 0.40 & 2101 & 0 & 0.054874 & 5076 & 0 & 0.776249 & 101256 & 166 & 10.215604 \\
\hline 0.39 & 2178 & 0 & 0.058049 & 6006 & 0 & 0.952096 & 135886 & 532 & 18.036749 \\
\hline 0.38 & 2292 & 0 & 0.060858 & 8738 & 1 & 1.139050 & - & - & - \\
\hline 0.37 & 2379 & 0 & 0.066534 & 11578 & 4 & 1.654584 & - & - & - \\
\hline$\overline{0.36}$ & 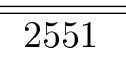 & $\overline{00}$ & 0.070770 & $\overline{24383}$ & $\overline{21}$ & 2.904537 & - & - & - \\
\hline 0.35 & 2717 & 0 & 0.075242 & 41542 & 96 & 5.194400 & - & - & - \\
\hline 0.34 & 2855 & 0 & 0.082025 & 63867 & 254 & 8.248176 & - & - & - \\
\hline 0.33 & 3170 & 0 & 0.091566 & - & - & - & - & - & - \\
\hline 0.32 & 3472 & 0 & 0.103102 & - & - & - & - & - & - \\
\hline 0.31 & 4022 & 0 & 0.120170 & - & - & - & - & - & - \\
\hline 0.30 & 4753 & 0 & 0.147201 & - & - & - & - & - & - \\
\hline 0.29 & 5733 & 0 & 0.182123 & - & - & - & - & - & - \\
\hline 0.28 & 7804 & 1 & 0.250031 & - & - & - & - & - & - \\
\hline 0.27 & 12224 & 4 & 0.379845 & - & - & - & - & - & - \\
\hline 0.26 & 20834 & 11 & 0.685417 & - & - & - & - & - & - \\
\hline 0.25 & 40398 & 78 & 1.364460 & - & - & - & - & - & - \\
\hline 0.24 & 96582 & 372 & 3.138895 & - & - & - & - & - & - \\
\hline
\end{tabular}

Tabela 4.4: Resultados dos experimentos da Reconstrução da Chave Secreta RSA módulo 3072.

\subsubsection{Experimentos para chaves de 4096 bits}

Os resultados dos experimentos realizados para chaves de 4096 bits corroboram: 


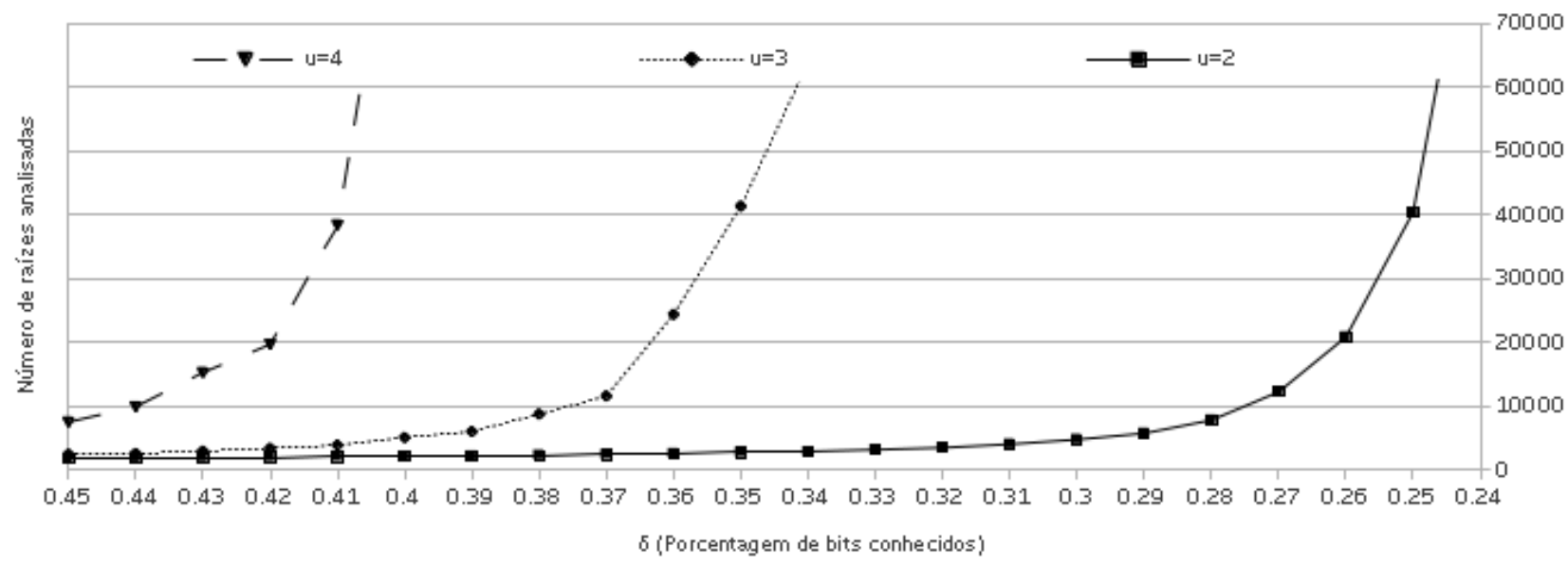

Figura 4.2: Comparação das médias da quantidade de raízes analisadas para chaves de 3072 bits.

- Um comportamento similar em relação aos valores de $\delta$

- Que a razão entre o número médio de raízes analisadas e o tempo médio de reconstrução das chaves é o dobro $\left(\frac{4096}{2048}=2\right)$

com relação aos experimentos realizados para chaves de 2048 bits.

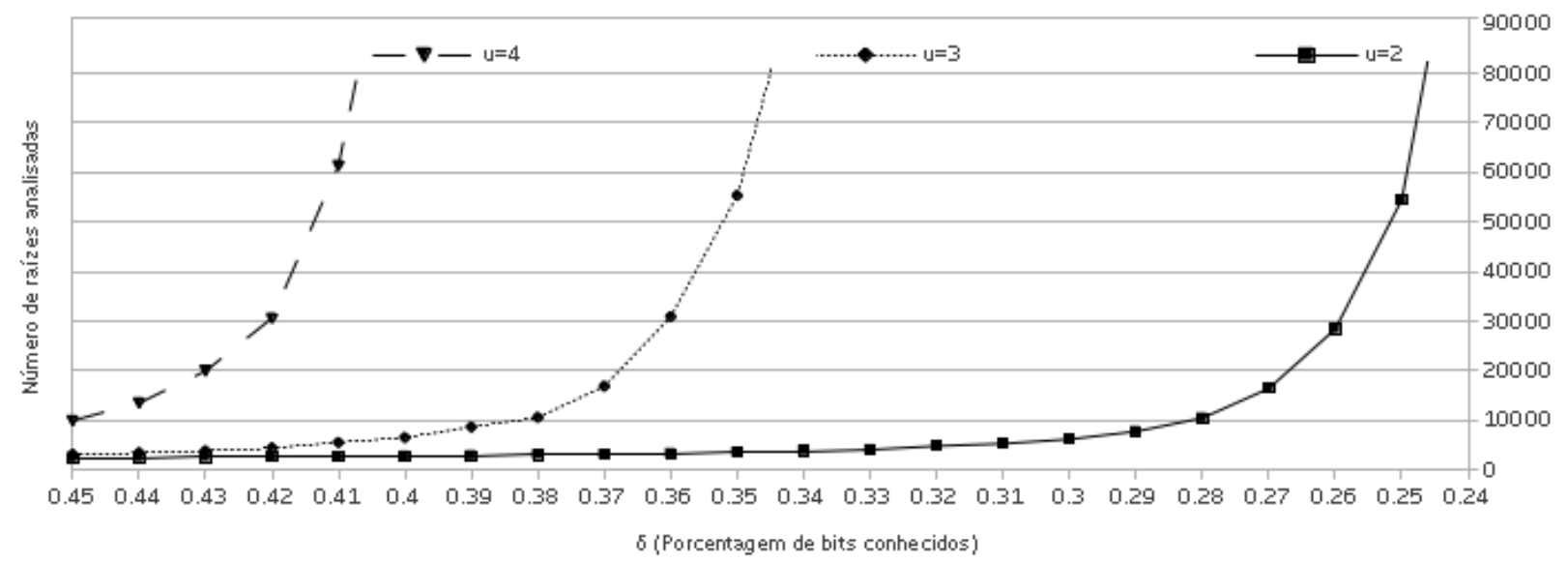

Figura 4.3: Comparação das médias da quantidade de raízes analisadas para chaves de 4096 bits. 


\begin{tabular}{|c|c|c|c|c|c|c|c|c|c|}
\hline & \multicolumn{3}{|c|}{ Criptossistema RSA básico } & \multicolumn{3}{|c|}{ Criptossistema RSA 3-primos } & \multicolumn{3}{|c|}{ Criptossistema RSA 4-primos } \\
\hline & \multicolumn{2}{|c|}{ \# exp. } & \multirow{2}{*}{$\begin{array}{l}\text { Tempo } \\
\text { Média }\end{array}$} & \multicolumn{2}{|c|}{ \# exp. } & \multirow{2}{*}{$\begin{array}{l}\text { Tempo } \\
\text { Média } \\
\end{array}$} & \multicolumn{2}{|c|}{ \# exp. } & Tempo \\
\hline$\delta$ & Média & $(>1 \mathrm{M})$ & & Média & $(>1 \mathrm{M})$ & & Média & $(>1 \mathrm{M})$ & Média \\
\hline 0.45 & 2435 & 0 & 0.086116 & 3146 & 0 & 0.490644 & 9969 & 0 & 2.223734 \\
\hline 0.44 & 2495 & 0 & 0.088632 & 3483 & 0 & 0.591384 & 13554 & 0 & 2.757694 \\
\hline 0.43 & 2556 & 0 & 0.092682 & 3853 & 0 & 0.675294 & 19964 & 11 & 4.321382 \\
\hline 0.42 & 2611 & 0 & 0.095857 & 4445 & 0 & 0.839434 & 30638 & 22 & 7.422394 \\
\hline 0.41 & 2726 & 0 & 0.100541 & 5568 & 0 & 0.974277 & 61439 & 89 & 12.667805 \\
\hline 0.40 & 2793 & 0 & 0.104544 & 6537 & 0 & 1.209522 & 120405 & 244 & 21.301735 \\
\hline 0.39 & 2876 & 0 & 0.109690 & 8686 & 1 & 1.768709 & 165025 & 707 & 34.527866 \\
\hline 0.38 & 3016 & 0 & 0.116647 & 10602 & 2 & 2.726161 & - & - & - \\
\hline 0.37 & 3144 & 0 & 0.125312 & 16877 & 4 & 3.986017 & - & - & - \\
\hline 0.36 & 3299 & 0 & 0.136678 & 30930 & 43 & 7.189447 & - & - & - \\
\hline 0.35 & 3540 & 0 & 0.145749 & 55364 & 147 & 11.668104 & - & - & - \\
\hline 0.34 & 3848 & 0 & 0.160085 & 104914 & 360 & 19.296354 & - & - & - \\
\hline 0.33 & 4084 & 0 & 0.176935 & - & - & - & - & - & - \\
\hline 0.32 & 4833 & 0 & 0.196175 & - & - & - & - & - & - \\
\hline 0.31 & 5318 & 0 & 0.230283 & - & - & - & - & - & - \\
\hline 0.30 & 6202 & 0 & 0.272566 & - & - & - & - & - & - \\
\hline 0.29 & 7775 & 0 & 0.357924 & - & - & - & - & - & - \\
\hline 0.28 & 10484 & 1 & 0.470226 & - & - & - & - & - & - \\
\hline 0.27 & 16448 & 8 & 0.720842 & - & - & - & - & - & - \\
\hline 0.26 & 28436 & 17 & 1.333051 & - & - & - & - & - & - \\
\hline 0.25 & 54493 & 117 & 2.621471 & - & - & - & - & - & - \\
\hline 0.24 & 126144 & 674 & 5.910489 & - & - & - & - & - & - \\
\hline
\end{tabular}

Tabela 4.5: Resultados dos experimentos da Reconstrução da Chave Secreta RSA módulo 4096. 


\section{Capítulo 5}

\section{Conclusões}

\subsection{Conclusões}

Com a análise feita no Capítulo 3, conseguimos calcular a porcentagem $\delta$ de bits corretos em $\tilde{s k}$ que é preciso para reconstruir a chave secreta RSA sk em tempo polinomial com uma probabilidade bastante grande. Onde o valor de $\delta$ é dado por

$$
\delta>2-2^{\frac{u+2}{2 u+1}}
$$

sendo $u$ o número de fatores primos do módulo $N$. Sabemos que enquanto o valor de $u$ for maior o valor mínimo de $\delta$ também é crescente, portanto a segurança vai se incrementando, mas tudo isso tem um limite. Se o atacante Carlos conseguir em algum ataque cold boot mais de um $59 \%$ de bits corretos em $\tilde{s k}$, ele vai conseguir quebrar o criptossistema RSA básico ou multi-primo em tempo polinomial sem importar o valor de $u$, isso devido a que

$$
\delta>\lim _{u \rightarrow \infty} 2-2^{\frac{u+2}{2 u+1}}=0.5858 .
$$

Em outras palavras, a complexidade de Algoritmo 2 para valores $\delta \geq 0.59$ sempre vai ser polinomial com uma probabilidade bastante grande. Mas essa contribuição é só teórica já que não sabemos realmente como é o seu comportamento experimental para quando o valor de $u$ tende ao infinito.

Com os resultados obtidos da análise do algoritmo feito em Seção 3.4.2 e dos experimentos no Capítulo 4 podemos asseverar que podemos reconstruir em tempo polinomial com uma grande probabilidade a chave secreta do criptossistema:

- RSA básico tendo uma porcentagem $\delta \geq 0.27$,

- RSA 3-primos tendo uma porcentagem $\delta \geq 0.37$,

- RSA 4-primos tendo uma porcentagem $\delta \geq 0.42$,

de bits corretos de $s k$ em $\tilde{s k}$ onde é clara a vantagem do criptossistema RSA multi-primo sobre o RSA básico com relação à quantidade de bits.

$$
2-2^{\frac{u+2}{2 u+1}}>2-2^{\frac{4}{5}} \text { para } u \geq 3
$$

Podemos concluir que enquanto o valor de $u$ for maior o Algoritmo 2 precisa de uma porcentagem maior de bits corretos $\left(2-2^{\frac{u+2}{2 u+1}}\right)$ em $\tilde{s k}$ para ter uma complexidade polinomial. Além disso o número máximo de vezes que o Algoritmo 2 deve ser executado também cresce e é dado pelo número de soluções possíveis para os valores de $\left\langle k, k_{1}, \ldots, k_{u}\right\rangle$. A seguir na Tabela 5.1 temos dados mais específicos que foram obtidos no percorrer desse trabalho e são comparados com o criptossistema RSA básico. 


\begin{tabular}{|c|c|c|c|}
\hline RSA & RSA básico & RSA 3-primos & RSA 4-primos \\
\hline$N=\prod_{i=1}^{\infty} r_{i}$ & $N=r_{1} r_{2}$ & $N=\prod_{i=1} r_{i}$ & $N=\prod_{i=1}^{4} r_{i}$ \\
\hline \multicolumn{4}{|c|}{ Número de soluções para $\left\langle k, k_{1}, \ldots, k_{u}\right\rangle$ sem conhecer o valor de $k$} \\
\hline$\alpha(u) \leq(e-2)^{u-1}$ & $\alpha(2) \leq(e-2)$ & $\alpha(2)<\alpha(3) \leq(e-2)^{2}$ & $\alpha(3)<\alpha(4) \leq(e-2)^{3}$ \\
\hline \multicolumn{4}{|c|}{ Número de bits mais significativos de $\tilde{d}$ que são precisos para calcular $k$} \\
\hline$n / u$ & $n / 2$ & $n / 3$ & $n / 4$ \\
\hline \multicolumn{4}{|c|}{ Número de soluções para $\left\langle k_{1}, \ldots, k_{u}\right\rangle$ conhecendo o valor de $k$} \\
\hline$\beta(u) \leq 2(e-2)^{u-2}$ & $\beta(2)=2$ & $\beta(3) \leq 2(e-2)$ & $\beta(4) \leq 2(e-2)^{2}$ \\
\hline \multicolumn{4}{|c|}{ Reconstrução da Chave Secreta RSA em tempo polinomial } \\
\hline$\delta>2-2^{\frac{u+2}{2 u+1}}$ & $\delta \geq 2-2^{\frac{4}{5}} \approx 0.27$ & $\delta \geq 2-2^{\frac{5}{7}} \approx 0.37$ & $\delta \geq 2-2^{\frac{6}{9}} \approx 0.42$ \\
\hline
\end{tabular}

Tabela 5.1: Comparação entre os criptossistemas RSA básico, 3-primos e 4-primos.

Em 2012, Jon Kogure fez a análise de reconstrução da chave secreta sk do criptossistema RSA multi-potência ${ }^{1}$ com bits aleatórios descrito em [KKY12]. Os resultados de Kogure mostram que temos uma única vantagem com respeito à reconstrução da chave secreta do RSA básico. Essa vantagem está relacionada com o cálculo de valor da variável $k$ o qual pela estrutura do RSA multi-potência só permite seu cálculo com os $\frac{n}{m+1}$ MSB do expoente de decriptação $d$. Em outras palavras, o atacante Carlos está limitado a usar os $\frac{n}{m+1}$ MSB onde esse valor é menor que os $\frac{n}{2}$ MSB que temos para calcular o valor de $k$ em um criptossistema RSA básico. Com relação aos outros tópicos mencionados na Tabela 5.2 temos que o criptossistema RSA multi-potência é equivalente ao criptossistema RSA básico. Com esses resultados podemos concluir que o criptossistema RSA multi-primo oferece mais segurança que os criptossistemas RSA básico e multi-potência com relação à reconstrução da sua chave secreta com bits aleatórios.

\begin{tabular}{|c|c|c|c|c|}
\hline \multicolumn{4}{|c|}{ Criptossistema RSA } \\
\hline RSA multi-potência & & RSA básico & & RSA multi-primo \\
\hline$N=r_{1}^{m} r_{2}$ & & $N=r_{1} r_{2}$ & & $N=\prod_{i=1}^{u} r_{i}$ \\
\hline$m \geq 2$ & $>$ & $m=1$ & & - \\
\hline- & & $u=2$ & $<$ & $u \geq 3$ \\
\hline \hline
\end{tabular}

Número de soluções para $\left\langle k, k_{1}, \ldots, k_{u}\right\rangle$ sem conhecer o valor de $k$

\begin{tabular}{|l|l|l|l|l|}
$\leq(e-2)$ & $\equiv$ & $\alpha(2) \leq(e-2)$ & $<$ & $\alpha(u) \leq(e-2)^{u-1}$ \\
\hline
\end{tabular}

Número de bits mais significativos de $\tilde{d}$ que são precisos para calcular $k$

\begin{tabular}{|c|c|c|c|c|}
\hline$n /(m+1)$ & $>$ & $n / 2$ & $<$ & $n / u$ \\
\hline \hline Número de soluções para $\left\langle k_{1}, \ldots, k_{u}\right\rangle$ conhecendo o valor de $k$ \\
\hline 2 & $\equiv$ & $\beta(2) \leq 2$ & $<$ & $\beta(u) \leq 2(e-2)^{2}$ \\
\hline \hline \multicolumn{6}{|c|}{ Reconstrução da Chave Secreta RSA em tempo polinomial } \\
\hline$\delta \geq 2-2^{\frac{4}{5}} \approx 0.27$ & $\equiv$ & $\delta \geq 2-2^{\frac{4}{5}} \approx 0.27$ & $<$ & $\delta>2-2^{\frac{u+2}{2 u+1}}$ \\
\hline
\end{tabular}

Tabela 5.2: Comparação entre os criptossistemas RSA multi-potência, básico e multi-primo.

\footnotetext{
${ }^{1}$ Variante do criptossistema RSA onde o modulo $N$ está dado por $N=r_{1}^{m} r_{2}$ com $m \geq 2$. A vantagem dessa variante é seu processo rápido de decriptação.
} 


\subsection{Recomendações}

Sabemos que temos duas representações da chave secreta segundo o PKCS \# 1 e que a chave secreta $s k$ está dado pela união de ambos. Um método para evitar que o atacante Carlos consiga reconstruir a chave secreta é o uso da primeira representação para o processo de decriptação dado por $s k\langle N, d\rangle$. Portanto, o atacante Carlos só vai obter uma chave secreta $\tilde{s} k\langle N, \tilde{d}\rangle$ onde em $\tilde{d}$ temos uma porcentagem $\delta$ de bits corretos de $d$. E segundo a análise do Algoritmo 2 (veja Subseção 3.4.1) temos que a probabilidade de ser polinomial é bastante grande quanto o valor esperado de raízes incorretas geradas por uma raiz incorreta é menor que $1(\mathbb{E}[B]<1)$. Portanto procedemos a calcular $\mathbb{E}[B]$.

\begin{tabular}{|c||c|c|c|c|}
\hline$d[j+\tau(k)]$ & $\left(c_{1}, c_{2}\right)$ & $\left(c_{1}, \overline{c_{2}}\right)$ & $\left(\overline{c_{1}}, c_{2}\right)$ & $\left(\overline{c_{1}}, \overline{c_{2}}\right)$ \\
\hline \hline desconhecido & $2^{u-1}$ & $2^{u-1}$ & $2^{u-1}$ & $2^{u-1}$ \\
conhecido & $2^{u-1}$ & 0 & $2^{u-1}$ & 0 \\
\hline
\end{tabular}

Tabela 5.3: Número de raízes incorretas geradas a partir de uma raiz incorreta quando temos só uma porcentagem de bits conhecidos de $d$.

O número de raízes incorretas geradas a partir de uma raiz incorreta quanto só temos conhecimento de alguns bits de $d$ são mostrados na Tabela 5.3, esses dados forma tomados da Tabela 3.4 tendo em consideração o valor $h=u$ já que não temos conhecimentos de nenhum bit dos fatores primos de $N$. Portanto procedemos a calcular o valor esperado de $B$.

$$
\begin{aligned}
\mathbb{E}[B]= & 2^{u-1} P(\bar{d})\left[P\left(c_{1}\right) P\left(c_{2}\right)+P\left(c_{1}\right) P\left(\overline{c_{2}}\right)+P\left(\overline{c_{1}}\right) P\left(c_{2}\right)+P\left(\overline{c_{1}}\right) P\left(\overline{c_{2}}\right)\right]+ \\
& 2^{u-1} P(d)\left[P\left(c_{1}\right) P\left(c_{2}\right)+P\left(\overline{c_{1}}\right) P\left(c_{2}\right)\right]
\end{aligned}
$$

Onde substituindo as probabilidades $P\left(c_{1}\right), P\left(\overline{c_{1}}\right), P\left(c_{1}\right), P\left(\overline{c_{1}}\right), P(d)$ e $P(\bar{d})$ vamos obter

$$
\begin{aligned}
\mathbb{E}[B] & =2^{u-1}(1-\delta)+2^{u-1} \delta \frac{1}{2} \\
& =2^{u-1} \frac{(2-\delta)}{2} \\
& =2^{u-2}(2-\delta) .
\end{aligned}
$$

Já determinado o $\mathbb{E}[B]$, então podemos afirmar que a reconstrução da chave secreta $s k$ vai acontecer em tempo polinomial se temos que

$$
\begin{aligned}
\mathbb{E}[B]=2^{u-2}(2-\delta) & <1 \\
(2-\delta) & <2^{2-u} \\
\delta & >2-2^{2-u}
\end{aligned}
$$

Onde podemos afirmar que

$$
\delta>2-2^{2-u} \geq 1 \text { para } u \geq 2 .
$$

Em conclusão, o atacante Carlos vai precisar de uma porcentagem $\delta>1$ de bits corretos em $d$ para reconstruir a chave secreta em tempo polinomial. Em outras palavras, não é possível a reconstrução da chave secreta em tempo polinomial, tendo bits aleatórios de $d$ e usando o método apresentado nesse trabalho.

\subsection{Trabalhos Futuros e Problemas Abertos}

Nesta seção apresentamos os trabalhos que podem complementar o trabalho desenvolvido. 
- Fatoração de um inteiro $N u$-primos tendo bits aleatórios de só $u-1$ ou menos primos (problema aberto)

Sabemos pela trabalho desenvolvido em [HS09], que não é possível a fatoração do inteiro $N$ 2-primos, só tendo bits aleatórios de $p$. Isso devido a que o desconhecimento total de $q$ faz do algoritmo, apresentado em $[\mathrm{TV}]$, de complexidade exponencial $(\mathbb{E}[B]>1)$. Em outras palavras, não é possível a fatoração do inteiro $N$ em tempo polinomial. Fazendo a mesma análise para um inteiro $N u$-primos, podemos concluir que não é possível a fatoração de $N$ $u$-primos em tempo polinomial só tendo uma fração de bits aleatórios de $u-1$ ou menos primos.

\section{- Diminuir a complexidade do algoritmo de reconstrução (problema aberto)}

Sabemos que o algoritmo de reconstrução é de força bruta, ou seja estamos testando todas as possíveis soluções para depois escolher a melhor, o qual não é prático. Portanto seria interessante o uso de reticulados(lattice reduction) ou alguns outros métodos para poder diminuir a complexidade do algoritmo.

- Usar toda a chave secreta $\tilde{s k}\left\langle\tilde{d}, \tilde{r_{1}}, \tilde{r_{2}}, \tilde{d_{1}}, \tilde{d_{2}}, \tilde{r_{2}}{ }^{-1},\left\langle\tilde{r_{3}}, \tilde{d_{3}}, \tilde{t_{3}}\right\rangle, \ldots,\left\langle\tilde{r_{u}}, \tilde{d_{u}}, \tilde{t_{u}}\right\rangle\right\rangle$ na execução do algoritmo de reconstrução (problema aberto)

Como podem ser usados a fração de bits corretos das variáveis $\left\langle{\tilde{r_{2}}}^{-1}, \tilde{t_{3}}, \ldots, \tilde{t_{u}}\right\rangle$ de $\tilde{s k}$ para a reconstrução da chave secreta? 


\section{Apêndice A}

\section{Teoria de Números}

\section{A.1 Divisibilidade e Congruências}

\section{A.1.1 Divisibilidade}

O conjunto dos inteiros ..., -3, -2, -1, - , 1, 2, 3, .. é denotado pelo símbolo $\mathbb{Z}$.

Definição 1. Sejam a e b inteiros. Então a divide $b$ se existe um inteiro $c$ tal que $b=a c$. a divide $b$ é denotado por a $\mid b$ e poder ser dito como a é um divisor de $b$, ou a é um fator de $b$.

Definição 2. Um inteiro c é um divisor comum de a e b se $c \mid a$ e $c \mid b$.

Definição 3. Um inteiro positivo d denotado por mdc(a,b) é o máximo divisor comum dos inteiros a e b se d é um divisor comum de a e b e sempre que c|a e c $\mid b$ então $c \mid d$, a exceção de $m d c(0,0)=0$.

Definição 4. Dois inteiros a e b são ditos relativamente primos ou coprimos se $m d c(a, b)=1$.

Definição 5. Um inteiros $p \geq 2$ é dito primo, se seus únicos divisores positivos são 1 e p. Caso contrário $p$ é chamado composto.

Definição 6. Seja $N \geq 2$ um inteiro natural. Podemos escrever $N$ de uma única forma como um produto $N=r_{1} r_{2} \ldots r_{u}$ onde $u \geq 1$ é um natural e $r_{1} \leq r_{2} \leq \ldots \leq r_{u}$ são primos.

\section{A.1.2 Congruência}

Se $a$ e $p$ são inteiros e $p>0$, o resto de $a$ dividido por $p$ é o menor inteiro $r \geq 0$ que difere de $a$ por um múltiplo de $p$ e será representado por $a \bmod p$ ( $a$ módulo $p$ ). Exemplo: $6,36,46$ e -4 possuem resto 6 quando são divididos por 10. Escrevemos $a=q b+r$ ou $a \equiv(r \bmod b)$, onde o inteiro $q$ é chamado é chamado quociente.

Carl F. Gauss em 1801 definiu que $a$ e $b$ são congruentes ou equivalentes módulo $p$ se $p \mid(a-b)$ para $p>0$, ou seja, $a-b=k p$ para algum inteiro $k$ [Rhe94]. Observe que isso é o mesmo que dizer que $a$ e $b$ são congruentes módulo $p$ se possuem o mesmo resto sobre $p$, assim $6,36,46,-4$ são congruentes módulo 10. Observe ainda, que existem apenas $p$ inteiros que não são equivalentes módulo $p$, pois o resto da divisão por $p$ é tal que $0 \leq r \leq p-1$. Definimos então:

Definição 7. O conjunto dos inteiros módulo $p$ é denotado por $\mathbb{Z}_{p}$. Observe que $\mathbb{Z}_{p}$ forma o conjunto dos inteiros 0, 1, 2, ..., p-1 onde a adição, a subtração e a multiplicação são executadas módulo p.

Definição 8. O conjunto de todos os elementos em $\mathbb{Z}_{p}$ que são relativamente primos a p é denotado por $\mathbb{Z}_{p}^{*}$. 


\section{A.1.3 Algoritmo de Euclides Estendido}

\section{Algoritmo de Euclides}

Como foi mencionado antes, o máximo divisor comum de dois inteiros $a$ e $b$, é o maior inteiro $d$ que pode ser dividir $a$ e $b$. O valor de $d$ pode ser calculado eficientemente pelo algoritmo de Euclides (Veja o Algoritmo 3).

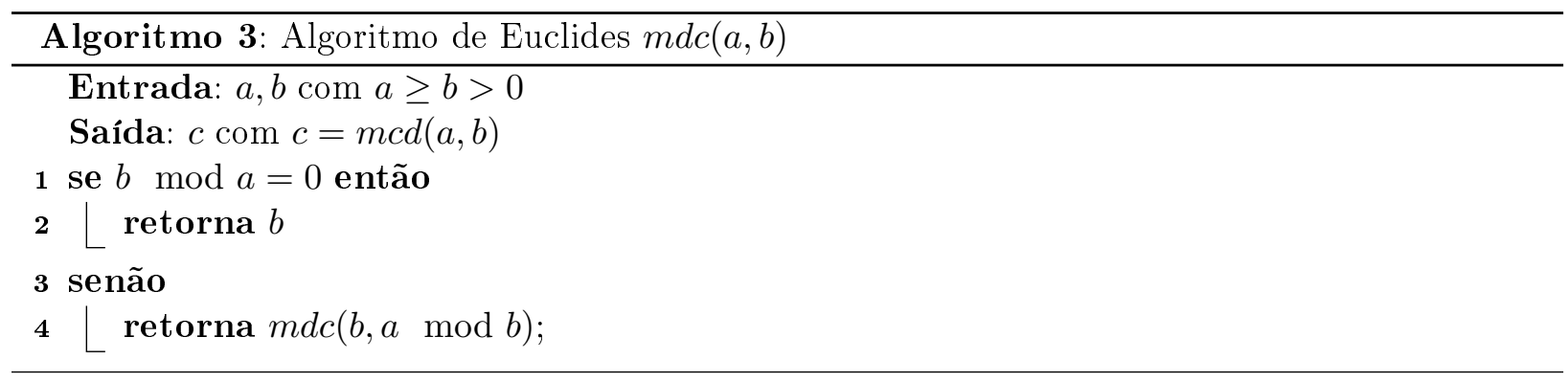

\section{Algoritmo de Euclides Estendido}

Para dois inteiros $a$ e $b$ existem inteiros $X$ e $Y$ onde $a X+b Y=m d c(a, b)$. Uma simples modificação do algoritmo de Euclides, chamado o algoritmo de Euclides Estendido, pode ser usado para calcular $X, Y$ e $m d c(a, b)$; veja o Algoritmo 4.

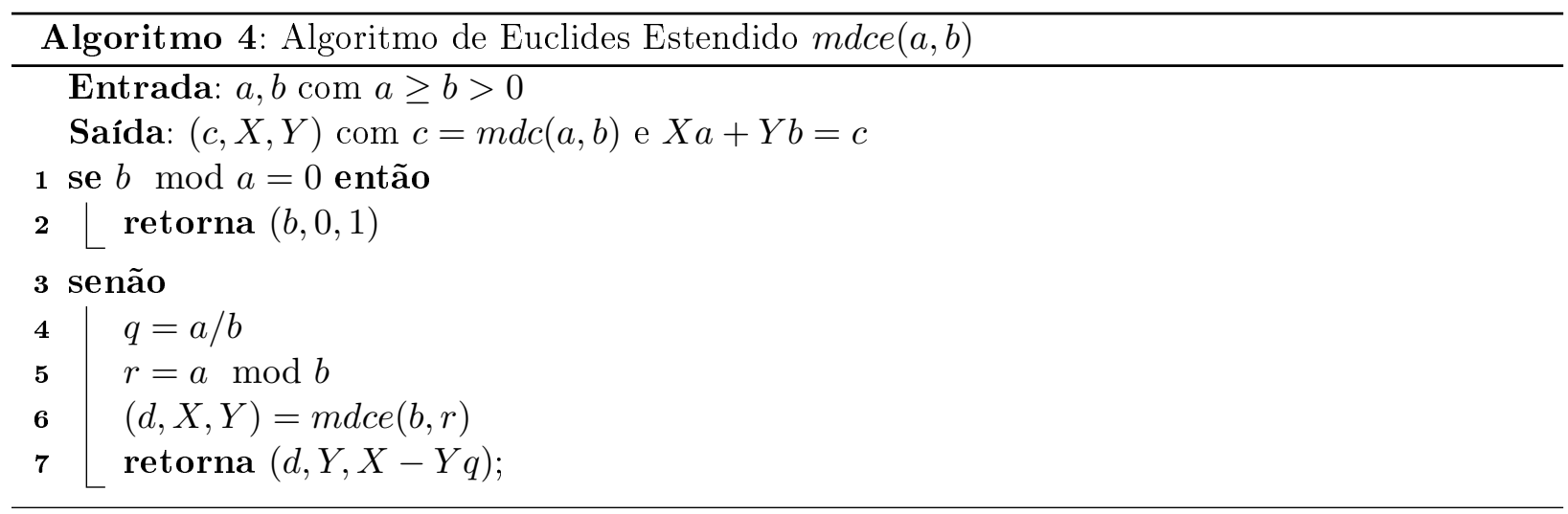

A demostração da corretude dos algoritmos de Euclides e Euclides Estendido podem ser encontrado em [KL08].

\section{A.1.4 Inversa Multiplicativa Módulo}

Seja $a \in \mathbb{Z}_{N}$. A inversa multiplicativa de $a$ módulo $N$ é um inteiro $x \in \mathbb{Z}_{N}$ tal que $a x \equiv 1$ $\bmod N$. Se tal $x$ existir ele é único, neste caso dizemos que $a$ é inversível e sua inversa é denotada por $a^{-1}$. Sabemos que $a$ é inversível se e somente se $m d c(a, N)=1$, e podemos calcular a inversa de $a$ pelo algoritmo de Euclides-Estendido. O resultado de aplicar o Algoritmo 4 a os inteiros $a$ e $N$ é $(\operatorname{mdc}(a, N), X, Y)$, onde aplicando módulo $\mathrm{N}$ temos que

$$
\begin{aligned}
a X & =m d c(a, N) \bmod N & \text { sabemos que } \operatorname{mdc}(a, N)=1 \\
X & =a^{-1} \bmod N . &
\end{aligned}
$$

Em outras palavras, o valor da inversa multiplicativa de $a$ está dado pelo valor $X$ calculado pelo algoritmo de Euclides Estendido. 


\section{A.1.5 Grupo e Anel}

As semelhanças entre as relações de congruência módulo $p$ e igualdade não são mero fruto do acaso, ambas são instâncias de relações de equivalências em $\mathbb{Z}$. Para entender melhor vamos definir os seguinte conceitos.

\section{Grupo}

Um grupo $\left(\mathbb{G},{ }^{*}\right)$ é um conjunto $\mathbb{G}$ com uma operação binária * (produto) que satisfaz os seguintes três axiomas:

1. (Associatividade) $\forall a, b, c \in \mathbb{G}$, temos $a *(b * c)=(a * b) * c$.

2. (Existência de elemento neutro) Existe um elemento $0 \in \mathbb{G}$, chamado identidade, tal que $\forall a \in \mathbb{G}$ temos que $a * 0=0 * a=a$.

3. (Existência de inverso) $\forall a \in \mathbb{G}$ existe um elemento $a^{-1} \in \mathbb{G}$, chamado o inverso de $a$, tal que $a * a^{-1}=a^{-1} * a=1$.

Se além dos três axiomas acima, o grupo $\mathbb{G}$ satisfaz

1. (Comutatividade) $\forall a, b \in \mathbb{G}$ temos que $a * b=b * a$.

então $\mathbb{G}$ é chamado de grupo abeliano.

Um exemplo é o grupo $(\mathbb{Z},+)$ ou seja, o grupo formado pelos inteiros $\mathbb{Z}$ com a operação soma usual. Este grupo é conhecido como grupo aditivo, a identidade é representada por 0 e o inverso de $a$ é representada por $-a$.

\section{Anel}

Um Anel $\left(\mathbb{A},+,{ }^{*}\right)$ é um conjunto $\mathbb{A}$ com duas operações binárias: $+($ soma $)$ e $\left(^{*}\right)$ produto satisfazendo axiomas que abstraem as propriedades usuais dos inteiros (por exemplo). Estes axiomas são:

1. $(\mathrm{A},+)$ é um grupo abeliano com elemento neutro 0 .

2. (Associativa do produto) $\forall a, b, c \in \mathbb{A}$ temos que $(a * b) * c=a *(b * c)$.

3. (Elemento neutro do produto) Existe um elemento $1 \in \mathbb{A}$ tal que $a * 1=a * 1=a \forall a \in \mathbb{A}$.

4. (Distributividade) $\forall a, b, c \in \mathbb{A}$ temos que $a *(b+c)=(a * b)+(a * c)$ e $(b+c) * a=(b * a)+(c * a)$.

Se $a * b=b * a$ para todo $a, b \in \mathbb{A}$, dizemos que o anel é comutativo o conjunto $\mathbb{Z}$ dos inteiros com as operações de soma e multiplicação usuais. Um outro anel importante ao nosso trabalho é o conjunto $\mathbb{Z}_{N}$ com a soma e multiplicação módulo $N$, pois é sobre este anel que o criptossistema RSA atua. Por outro lado, se em um anel comutativo $\mathbb{A}$ todo elemento não nulo possui inverso multiplicativo então dizemos que o anel $\mathbb{A}$ forma um corpo, ou seja, um anel comutativo no qual todos os elementos não nulos possuem inverso multiplicativo (veja na secção A.1.4).

Definição 9. $O$ anel $\mathbb{Z}_{p}$ é um corpo se, e somente se, p é primo.

\section{A.1.6 A Função Euler e o Teorema de Euler-Fermat}

Dizemos que um conjunto de $N$ números inteiros $a_{1}, a_{2}, \ldots, a_{N}$ forma um sistema completo de restos (scr) módulo $N$ se:

$$
\left\{a_{1}, a_{2}, \ldots, a_{N}\right\}=\mathbb{Z}_{N}
$$


isto é, que os $a_{i}$ representam todas as classes de congruência módulo $N$. Por exemplo, $0,1, \ldots, N-$ 1 formam um scr módulo $N$. Equivalentemente podemos dizer que $a_{1}, a_{2}, \ldots, a_{N}$ formam um scr módulo $N$ se, e somente se, $a_{i} \equiv a_{j} \bmod N$ implicar $i=j$.

De igual forma, dizemos que os números inteiros $b_{1}, b_{2}, \ldots, b_{\phi(N)}$ formam um sistema completo de invertíveis (sci) módulo $N$ se

$$
\left\{b_{1}, b_{2}, \ldots, b_{\phi(N)}\right\}=\mathbb{Z}_{N}^{*}
$$

onde $\phi(N)$ representa o número de elementos de $\mathbb{Z}_{N}^{*}$. Em outras palavras, $b_{1}, b_{2}, \ldots, b_{\phi(N)}$ formam um sci módulo $N$ se, e somente se, representam todas as classes de congruência invertíveis módulo $N$ ou, equivalentemente, $\operatorname{mdc}\left(b_{i}, N\right)=1$ para todo $i$ e $b_{i} \equiv b_{j} \bmod N$ implicar $i=j$. O conjunto $\{k \in \mathbb{Z} \mid 1 \leq k \leq N$ e $\operatorname{mdc}(N, k)=1\}$ é um sci módulo $N$.

Teorema 1. $\mathbb{Z}_{N}^{*}$ é fechado sobre a multiplicação módulo $N$ (veja em [Ter0o]).

Definição 10. A função $\phi(N)=\left|\mathbb{Z}_{N}^{*}\right|$ é chamada de função phi de Euler. Em particular para $N=\prod_{i=1}^{u} r_{i}^{\alpha_{i}}$ onde $N$ é o produto de primos distintos $\phi(N)=\prod_{i=1}^{u}\left(r_{i}^{\alpha_{i}}-r_{i}^{\alpha_{i}-1}\right)=N \prod_{i=1}^{u}\left(1-\frac{1}{r_{i}}\right)$.

Teorema 2 (Euler-Fermat). Sejam $r_{1}, r_{2} \ldots r_{u}, u$ primos distintos e $N=\prod_{i=1}^{u} r_{i}$. Então para todo $a \in \mathbb{Z}_{N}^{*}$ temos $a^{\phi(N)} \equiv 1 \bmod N$.

Através do Teorema de Euler chegamos ao seguinte corolário.

Corolário 1. Sejam $r_{1}, r_{2} \ldots r_{u}$, u primos distintos e $N=\prod_{i=1}^{u} r_{i}$. Então para todo a $\in \mathbb{Z}_{N}^{*}$ e um inteiro positivo $k$ temos $a^{k \phi(N)+1} \equiv a \bmod N$. Pois, $a^{k \phi(N)+1}=a^{k \phi(N)} a=1^{k} a=a \bmod N$.

\section{A.1.7 Polinômios}

Dado um anel comutativo $\mathbb{K}$, definimos o anel comutativo $\mathbb{K}[x]$ como o conjunto das expressões da forma $f(x)=a_{0}+a_{1} x+a_{2} x^{2}+a_{3} x^{3}+\ldots+a_{m} x^{m}$ com $a_{i} \in \mathbb{K}$, chamados de polinômios com coeficientes em $\mathbb{K}$. A soma e produto em $\mathbb{K}[x]$ são definidos da maneira usual: dados $f(x)=\sum_{i} a_{i} x^{i}$ e $g(x)=\sum_{i} b_{i} x^{i}$ elementos de $\mathbb{K}[x]$ temos

$$
\begin{aligned}
f(x)+g(x) & =\sum_{i}\left(a_{i}+b_{i}\right) x^{i} \\
f(x) . g(x) & =\sum_{i} c_{k} x^{k} \text { onde } c_{k}=\sum_{i+j=k} a_{i} b_{j}
\end{aligned}
$$

Definimos o grau $\operatorname{deg} f(x)$ de um polinômio $f(x)=a_{0}+a_{1} x+a_{2} x^{2}+a_{3} x^{3}+\ldots+a_{m} x^{m}$ como o maior $i$ tal que $a_{i} \neq 0$ : o grau do polinômio nulo 0 é definido como $-\infty$. Tal convenção visa a tornar válidas as seguintes identidades para todos os polinômios $f(x), g(x) \in \mathbb{K}[x]$ :

$$
\begin{gathered}
\operatorname{deg}(f(x) \cdot g(x))=\operatorname{deg} f(x)+\operatorname{deg} g(x) \mathrm{e} \\
\operatorname{deg}(f(x)+g(x)) \leq \max \{\operatorname{deg} f(x), \operatorname{deg} g(x)\}
\end{gathered}
$$

O Coeficiente do termo de maior grau de um polinômio é chamado de coeficiente líder. Um polinômio cujo coeficiente líder é igual a 1 é chamado de mônico.

Observe que nas definições acima $x$ é um simbolo formal e não um elemento de $\mathbb{K}$. A pesar disso, cada polinômio $f(x)=a_{0}+a_{1} x+a_{2} x^{2}+a_{3} x^{3}+\ldots+a_{m} x^{m}$ define uma função polinomial

$$
\begin{aligned}
f: \mathbb{K} & \rightarrow \mathbb{K} \\
c & \mapsto f(c)=a_{0}+a_{1} c+a_{2} c^{2}+a_{3} c^{3}+\ldots+a_{m} c^{m}
\end{aligned}
$$


também chamada de $f$. A distinção entre um polinômio e uma função polinomial é bem ilustrada pelo polinômio $f(x)=x p-x \in \mathbb{Z}_{\mathbb{N}}[x]$ : este polinômio é não nulo pois seus coeficientes são não nulos mas para todo $c \in \mathbb{Z}_{p}$ temos $f(c)=0$ pelo pequeno teorema de Euler-Fermat (veja o teorema 2). Dado um polinômio $f(x) \in \mathbb{K}[x]$, qualquer $c \in \mathbb{K}$ tal que $f(c)=0$ é chamado de raiz ou zero de $f(x)$.

Definição 11. Seja $\mathbb{K}$ um corpo. Dados polinômios $f(x), g(x) \in \mathbb{K}[x]$, com $g(x) \neq 0$, existem $q(x), r(x) \in \mathbb{K}[x]$ (chamados respectivamente de quociente e resto da divisão de $f(x)$ por $g(x)$ ), unicamente determinados, tais que

$$
f(x)=g(x) q(x)+r(x) \operatorname{com} \operatorname{deg} r(x)<\operatorname{deg} q(x) .
$$

Definição 12. Seja $\mathbb{K}$ um corpo, $f(x) \in \mathbb{K}[x]$ e $a \in \mathbb{K}$. Então

$$
x-a \mid f(x) \Longleftrightarrow f(a)=0 .
$$

Definição 13. Seja $\mathbb{K}$ um corpo. Um polinômio $f(x) \in \mathbb{K}[x]$ não nulo de grau $m$ tem no máximo $m$ raizes em $\mathbb{K}$

\section{A.2 Equações Módulo $m$}

Neste seção serão estudados equações do tipo

$$
a x=b \bmod m
$$

na variável $x$, onde $f(x)$ é um polinômio com coeficientes inteiros.

\section{A.2.1 Equações Lineares Módulo $m$}

Definição 14. Se $m d c(a, m)=1$, como a é inversivel módulo $m$, a equação

$$
a x \equiv b \quad \bmod m
$$

tem solução única módulo $p$, dada por $x \equiv a^{\phi(p)-1} b \bmod m$ (utilizando o teorema de Euler-Fermat para encontrar o inverso de $a \in \mathbb{Z}_{m}$

Definição 15. A congruência linear

$$
a x \equiv b \quad \bmod m
$$

admite solução se, e somente se, $m d c(a, m) \mid b$. Neste caso, há exatamente $m d c(a / m)$ soluções distintas módulo $m$.

Definição 16. Se $\operatorname{mdc}(a, p)=1$ e $m d c(b, p)=1$, então $a x+b y=c \bmod p$ tem exatamente $p$ soluções distintas.

Onde a definição anterior pode ser generalizada na seguinte definição.

Definição 17. Se $\operatorname{mdc}\left(a_{i}, p\right)=1$ para $1 \leq i \leq h$, então $\sum_{i=1}^{h} a_{i} x_{i}=b \bmod p$ tem exatamente $p^{h-1}$ soluçôes distintas.

\section{Teorema Chinês dos Restos - TCR}

O teorema chinês do resto é um algoritmo que permite resolver sistemas de congruências lineares. Possui este nome porque um dos primeiros lugares em que apareceu foi no livro Manual de Aritmética de Mestre Sun, escrito em torno de 100 D.C, pelo matemático Sun-Tsu. O matemático resolveu o problema de calcular inteiros $x$ que possuem restos $2,3,2$ quando divididos 
respectivamente por $3,5,7$. A solução desse problema está dada por $x=23$ ou especificamente $x=23+105 s$ para qualquer inteiro $s$ (observe que 105 é o produto de 3,5 e 7 ).

O problema pode ser enunciado da seguinte forma: Seja os inteiros $r_{1}, r_{2}, \ldots, r_{u}$ são relativamente primos entre si, então o sistema de congruências

$$
\begin{aligned}
& x \equiv a_{1} \quad \bmod r_{1} \\
& x \equiv a_{2} \quad \bmod r_{2} \\
& \text { : } \\
& x \equiv a_{u} \quad \bmod r_{u}
\end{aligned}
$$

Admite solução, que é única módulo $N=r_{1} r_{2} \ldots r_{u}$.

A solução do sistema para $u \geq 2$ é descrita da seguinte forma. Sejam $N_{1}=\left(\prod_{i=1}^{u} r_{i}\right) / r_{1}, \ldots, N_{u}=$ $\left(\prod_{i=1}^{u} r_{i}\right) / r_{u}$. Pelo algoritmo de Euclides Estendido (veja [KL08] secção B.1.2) calculamos os únicos inteiros

$$
\begin{array}{cc}
N_{1}^{\prime} & =N_{1}^{-1} \bmod r_{1}, \\
\vdots & \\
N_{k}^{\prime} & =N_{u}^{-1} \bmod r_{u} .
\end{array}
$$

Como cada $r_{i}$ é co-primo ao seu correspondente $N_{i}$ e utilizando o Teorema Chinês do Resto como foi mostrado, obtemos :

$$
x=\sum_{i=1}^{u} a_{i} N_{i}\left(N_{i}^{-1} \bmod r_{i}\right) \bmod N .
$$

Enunciamos o resultado formalmente a seguir:

Teorema 3 (TCR). Suponha $r_{1}, \ldots, r_{u}$ inteiros positivos relativamente primos entre si e sejam $a_{1}$, $\ldots, a_{u}$ inteiros. O sistema de u congruências $x \equiv a_{i} \bmod n_{i}(1 \geq i \geq u)$ possui uma única soluçẫo módulo $N=n_{1} n_{2} \ldots n_{k}$ que é dada por

$$
x=\sum_{i=1}^{k} a_{i} N_{i} b_{i} \quad \bmod N
$$

onde $N_{i}=\frac{N}{r_{i}}$ e $b_{i}=N_{i}^{-1} \bmod n_{i}$, para $1 \leq i \leq u$.

\section{A.2.2 Congruências de Grau 2}

seja $p \geq 2$ um número primo e $a, b \in \mathbb{Z} \operatorname{com} a$ não divisível por $p$. Resolver a equação quadrática

$$
a x^{2}+b x+c \equiv 0 \quad \bmod p
$$

é o mesmo que resolver (completando quadrados)

$$
(2 a x+b)^{2} \equiv b^{2}-4 a c \quad \bmod p
$$

(Note que 2 e $a$ são invertíveis módulo $p$ ). Assim, estamos interessados em encontrar critérios de existência de soluções da equação

$$
(2 a x+b)^{2} \equiv Y \quad \bmod p
$$

onde $Y=b^{2}-4 a c$. Se a equação acima admite solução (i.e. se $Y$ é um quadrado perfeito no anel comutativo $\mathbb{Z}_{p}^{*}$ ) então dizemos que o $Y$ é um resíduo quadrado ou resto quadrático módulo $p$. Há 
exatamente $(p+1) / 2$ resíduos quadráticos módulo $p$, a saber

$$
0^{2}, 1^{2}, 2^{2}, 3^{2}, \ldots\left(\frac{p-1}{2}\right)^{2} \bmod p
$$

já que todo inteiro $d$ é congruente a $\pm i \bmod p$ para algum $i$ tal que $0 \leq i \leq \frac{p-1}{2}$, de modo que $d^{2}$ é congruente a um dos números da lista acima. Portanto a solução para a equação quadrática é dado por

$$
2 a x+b \equiv \pm i \quad \bmod p
$$

onde temos que $( \pm i)^{2}=Y \bmod p$.

Embora saibamos a lista completa de resíduos quadráticos, na prática pode ser difícil reconhecer se um número é ou não resíduo quadrado. Para o entendimento disso vamos a definir o Símbolo de Legendre.

Definição 18 (Simbolo de Legendre). Seja $p>2$ um número primo e a um inteiro qualquer. $O$ Símbolo de Legendre está definido

$$
\left(\frac{a}{p}\right)= \begin{cases}1 & \text { se } p \text { \a e a é um resíduo quadrado módulo } p \\ 0 & \text { se } p \mid a \\ -1 & \text { Caso contrário }\end{cases}
$$

Definição 19 (Critério de Euler). Seja $p>2$ um número primo e a um inteiro qualquer. Então

$$
\left(\frac{a}{p}\right) \equiv a^{\frac{p-1}{2}} \bmod p
$$

\section{A.3 Conceitos Extras}

Teorema 4. O número de combinaçôes possiveis de a elementos escolhendo só b elementos está dado por $\left(\begin{array}{l}a \\ b\end{array}\right)$ [CC98]

$$
C_{b}^{a}=\left(\begin{array}{l}
a \\
b
\end{array}\right)=\frac{a !}{b !(a-b) !}
$$

Teorema 5. Em matemática, o binômio de Newton permite escrever na forma canônica o polinômio correspondente à potência de um binômio [Bin]. O binômio de Newton está definido da seguinte forma

$$
(x+y)^{a}=\sum_{b=0}^{a}\left(\begin{array}{l}
a \\
b
\end{array}\right) x^{a-b} y^{b} .
$$




\section{Apêndice B}

\section{Probabilidade}

\section{B.1 Experimentos Aleatórios}

Os primeiros estudos sob probabilidade foram motivados pela possibilidade de sucesso ou fracasso nos jogos de azar. A probabilidade é um mecanismo para estudar os eventos aleatórios, ou seja, operações cujo resultado não podem ser previstos com fiabilidade.

\section{B.1.1 Introdução}

Definição 20 (Experimento Aleatório ou Experimento). Um E.A. é qualquer operação cujo resultado não pode ser previsto com fiabilidade.

Exemplo:

1. Lançamento de uma moeda.

2. Lançamento de um dado.

3. Extração de uma carta de baralho de 52 cartas.

\section{B.1.2 Espaço Amostral e Eventos}

Definição 21 (Espaço Amostral). Um Espaço Amostral é o conjunto de todos os possíveis resultados relacionados a um experimento aleatório. Seu símbolo é $\Omega$ e se o espaço amostral tem um número finito de elementos ou um infinito numerável é chamado de discreto e se tem como elementos puntos de algum intervalo, então é chamado de contínuo.

Exemplo:

1. Lançamento de um dado. $\Omega=\{1,2,3,4,5,6\}$

2. Duração de um tubo fluorescente. $\Omega=\{t, t \geq 0\}$

Definição 22 (Evento). Um evento é um subconjunto $A$ de um Espaço Amostral $\Omega$, ou seja é um conjunto de resultados possiveis. Se o resultado do evento é um elemento de $\Omega$ dizemos que o evento evento A tem acontecido. Tudo subconjunto A é um evento, em particular $\Omega$ é um evento, chamado evento sucesso e o conjunto vazio $\not$, também é um evento chamado evento fracasso ou impossivel.

Exemplo:

1. $A=\{$ Obter um número impar no lançamento de um dado $\}$ $A=\{1,3,5\}$

2. $B=\{$ Obter ao menos uma cara no lançamento de uma moeda duas vezes $\}$ $B=\{c s, s c, c c\}$ 


\section{B.1.3 Probabilidades}

No momento de realização de um experimento aleatório sempre temos uma incerteza sob o resultado, o Conceito de probabilidade define uma medida numérica para ter uma ideia de qual fácil ou difícil pode um evento acontecer.

A ideia geral da probabilidade é frequentemente dividida em dois conceitos relacionados:

1. (Definição de Frequência - A posteriori) Seja um Experimento Aleatório repetível, ou seja, depois de uma tentativa é possível voltar ao estado inicial e repetir o experimento de jeito que o resultado não seja afetado por los resultados prévios. Se o experimento é feito $L$ vezes e o evento $A$ acontece $L_{A}$ das $L$ vezes, temos que a proporção de acontecimentos do evento $A$ en $L$ tentativas é igual a $\frac{L_{A}}{L}$. Se $L$ for grande esperamos que a proporção seja aproximadamente constante. Segundo ao conceito de frequência de probabilidade, a probabilidade do que o evento $A$ aconteça é o limite do cociente $\frac{L_{A}}{L}$ a medida que $L$ se faz infinitamente grande.

$$
P(A)=\lim _{L \rightarrow \infty} \frac{L_{A}}{L}
$$

2. (Definição clássica - A priori) Seja um Espaço Amostral $\Omega$ com $L$ resultados possíveis (todos eles equiprováveis). Seja o evento $A \mathrm{com} L_{A}$ resultados favoráveis, a probabilidade do evento $A$ é igual ao cociente:

$$
P(A)=\frac{\text { resultados favoráveis }}{\text { resultados possíveis }}=\frac{L_{A}}{L}
$$

\section{Propriedades}

1. Seja $A$ um evento e $\bar{A}$ seu evento complemento

$$
P(\bar{A})=1-P(A)
$$

2. Sejam $A$ e $B$ dois eventos quaisquer,

$$
P(A \cup B)=P(A)+P(B)-P(A \cap B)
$$

(Essa propriedade pode ser generalizada para $m$ eventos quaisquer)

\section{Probabilidade Condicional e Independência}

Supondo que estamos interessados em dois eventos $A$ e $B$, e temos uma informação adicional do que o evento $A$ tem acontecido. As vezes pode interessar a pregunta: Qual é a probabilidade que se verifique o evento $A$ dado que o evento $A$ já tem acontecido? Esse tipo de problema pode ser acatado a través da noção de probabilidade condicional.

Definição 23. Em um Experimento Aleatório, dados dos eventos $A$ e $B$, chama-se probabilidade condicional de $A$ dado que o evento $B$ tem acontecido a:

$$
P(A / B)=\frac{P(A \cap B)}{P(B)}
$$

sempre que $P(B)>0$.

Definição 24. Disse-se que dois eventos $A$ e $B$ são estatisticamente independentes se:

$$
P(A / B)=P(A)
$$


Intuitivamente, podemos ver como a verificação do evento $B$ não altera em absoluto a probabilidade do evento $A$. Portanto podemos asseverar que dois eventos são estatisticamente independentes se e só se:

$$
P(A \cap B)=P(A) \cdot P(B)
$$




\section{Apêndice C}

\section{Estatística}

\section{C.1 Variável Aleatória}

Temos visto como os resultados de um Experimento Aleatório não são necessariamente numéricos (obter cara ao lançar uma moeda, obter uma bola branca, ...). Por outro Jeito não temos por que estudar aqueles resultados (eventos) que não nos interessa. As variáveis aleatórias nós permitem centrar nossa atenção em aqueles eventos importantes de nosso estudo, os associando números.

\section{C.1.1 Variável Aleatória}

Dado um E.A com Espaço Amostral $\Omega$, se chama variável aleatória (v.a) del experimento a qualquer aplicação del E.M. no conjunto dos números reais, ou seja, a qualquer resultado del experimento é atribuído um número real.

$$
\begin{aligned}
X: \Omega & \rightarrow \mathbb{R} \\
A_{i} & \rightarrow X\left(A_{i}\right)=x_{i}
\end{aligned}
$$

É importante distinguir a variável aleatória dos valores que podem ser atribuídos a ela. As letras maiúsculas, tais como $X$ representam às v.a. enquanto as letras minúsculas representam os valores números que toma a v.a. $X$.

\section{Variável Aleatória Discreta}

Uma v.a. é discreta se e só se pode tomar uma quantidade numerável de valores.

Se a v.a. tem um número discreto de valores então é uma v.a. discreta. Por exemplo, número de caras obtidas ao lançar 5 vezes uma moeda. Também é discreto se tem um número infinitos numerável de valores. Por exemplo, el número de lançamentos de una moeda hasta obter cara.

\section{C.2 Função de Probabilidade de uma Variável Aleatória}

Definição 25. Seja uma v.a.d. X para um E.A., chama-se função de probabilidade dessa variável à aplicação onde a cada número $x_{i}$ faz corresponder uma probabilidade de sucesso $X=x_{i}$.

$$
\begin{aligned}
& \Omega \stackrel{X}{\rightarrow} \mathbb{R} \quad \stackrel{P_{x}}{\rightarrow}[0,1] \subseteq \mathbb{R} \\
& A_{i} \quad \rightarrow \quad X\left(A_{i}\right)=x_{i} \quad \rightarrow \quad P_{X}\left(x_{i}\right)=P\left(X=x_{i}\right)
\end{aligned}
$$

A função de probabilidade toma valor 0 em todos os demais valores da variável, que corresponde a sucessos impossiveis. 


\section{C.3 Distribuição de Probabilidade}

A distribuição da probabilidade contem toda a informação sob as propriedades de uma variável aleatória. $\mathrm{O}$ valor esperado de uma v.a. é apresentada com a finalidade de obter uma medida de centralização de uma distribuição de probabilidade. Se um mesmo E.A. é feito $l$, o valor esperado da v.a. é a medida dos valores quando $l$ tende ao infinito $l \rightarrow \infty$. A variância de uma v.a. é a medida da dispersão de uma distribuição de probabilidade. Intuitivamente, a variância pode se entender como o valor promédio que tomará o quadrado das desviações respeito ao valor esperado sob um número grande de repetições do experimento. Los momentos de uma v.a. ajudam a estudar o jeito da distribuição de probabilidade.

\section{C.3.1 Valor Esperado}

Definição 26. Dada uma v.a.d. $X$ com a função de probabilidade $P_{X}(x)$, se define o valor esperado de $X$ como:

$$
\mathbb{E}[X]=\sum_{x} x P_{x}(x)=\sum_{x} x P_{x}(X=x)
$$

O valor esperado também é conhecido como valor promédio e é representado como $\mu_{X}$. Tendo em conta a definição anterior é fácil demostrar que para duas variáveis aleatórias discretas $X$ e $Y$ temos que:

$$
\mathbb{E}[X+Y]=\mathbb{E}[X]+\mathbb{E}[Y]
$$

\section{C.3.2 Variância}

Definição 27. Dada uma v.a. X, define-se como a variância como o valor esperado do quadrado das desviaçôes com relação ao valor esperado.

$$
\operatorname{Var}(X)=\mathbb{E}\left[\left(X-\mu_{X}\right)^{2}\right]=\mathbb{E}\left[(X-\mathbb{E}[X])^{2}\right]
$$

O variância de uma v.a. é representado $\sigma_{X}^{2}$.

Se a v.a. é discreta temos

$$
\begin{aligned}
\sigma_{X}^{2}=\operatorname{Var}(X) & =\sum_{x}\left(x-\mu_{X}\right)^{2} P_{X}(x) \\
& =\sum_{x} x^{2} P_{X}(x)-\mu_{X}^{2} \\
& =\mathbb{E}\left[X^{2}\right]-\mathbb{E}[X]^{2}
\end{aligned}
$$

\section{C.3.3 Desigualdade de Chebyshev}

O teorema de Chebyshev o a desigualdade de Chebyshev proporciona um intervalo para a probabilidade de que uma v.a. fique longe de certo número de desviações estândar do valor esperado. A cota não sempre gera um intervalo reduzido; embora, requere-se muito poca informação da variável aleatória para gerar o intervalo, de fato, só precisamos conhecer o valor esperado e a desviação estândar.

Teorema 6. A probabilidade de que qualquer v.a. tenha um valor dentro das c desviações estândar do valor esperado é pelo menos de $1-\frac{1}{c^{2}}$; ou seja,

$$
P\left(\mathbb{E}[X]-c \sigma_{X}<X<\mathbb{E}[X]+c \sigma_{X}\right) \geq 1-\frac{1}{c^{2}}
$$


De fato, a desigualdade de Chebyshev pode ser escrita como:

$$
P\left(|X-\mathbb{E}[X]|<c \sigma_{X}\right) \geq 1-\frac{1}{c^{2}}
$$

ou bem, utilizando o complemento

$$
P\left(|X-\mathbb{E}[X]| \geq c \sigma_{X}\right) \leq \frac{1}{c^{2}}
$$

\section{C.3.4 Momentos}

Definição 28. Dada uma v.a. $X$, chama-se como momento de ordem $r$ com relação ao origem ao valor esperado da potencia r-ésima da variável.

$$
a_{r}=\mathbb{E}\left[X^{r}\right]
$$

Se a v.a. é discreta temos

$$
a_{r}=\mathbb{E}\left[X^{r}\right]=\sum_{x} x^{r} \cdot P_{X}(x)
$$

\section{C.4 Distribuição Conjunta de Duas V.A.}

No estudo conjunto de duas v.a. temos um estatístico que resulta muito útil: A covariância.

\section{C.4.1 Covariância}

Definição 29. Sejam $X$ e $Y$ duas variáveis com valor esperado $\mu_{X}$ e $\mu_{Y}$ respetivamente, chama-se covariância de $X$ e $Y$ ao valor esperado do produto das desviações repeito aos valores esperados.

$$
\begin{aligned}
\operatorname{Cov}(X, Y) & =\mathbb{E}\left[\left(X-\mu_{X}\right)\left(Y-\mu_{Y}\right)\right] \\
& =\mathbb{E}[X Y]-\mu_{X} \mu_{Y} \\
& =\mathbb{E}[X Y]-\mathbb{E}[X] \mathbb{E}[Y]
\end{aligned}
$$

\section{C.5 Coeficiente de Correlação de Pearson}

Definição 30. O coeficiente de correlação de Pearson entre duas v.a. X e Y está definido por

$$
\rho=\frac{\mathbb{E}[X-\mathbb{E}[X]] \mathbb{E}[Y-\mathbb{E}[Y]]}{\sqrt{\mathbb{E}\left[(X-\mathbb{E}[X])^{2}\right] \mathbb{E}\left[(Y-\mathbb{E}[Y])^{2}\right]}}=\frac{\operatorname{Cov}(X, Y)}{\sqrt{\operatorname{Var}[X] \operatorname{Var}[Y]}} .
$$

O coeficiente de correlação de Pearson $\rho$ é uma medida que mostra o comportamento das v.a. $X$ e $Y$ indicando a situação relativa dos seus eventos, em outras palavras, é um valor numérico que indica o grau de relação existente entre as duas v.a. e como elas estão relacionadas. O valor de $\rho$ varia nos limites de -1 a 1 , onde seu valor indica o grau de associação das v.a. $O$ valor 0 indica que não existe relação. $\mathrm{O}$ valores positivos indican uma correlação perfeita e os valores negativos indica uma correlação imperfeita (ver [CC98] e [Pea]).

Para nosso análise, precisamos a seguinte resultado. Sabemos que o valor de $\rho$ está entre -1 e 1 então podemos afirmar que

$$
\begin{aligned}
-1 & \leq \frac{\operatorname{Cov}(X, Y)}{\sqrt{\operatorname{Var}[X] \mathbb{V a r}[Y]}} \leq 1 \\
-\sqrt{\operatorname{Var}[X] \operatorname{Var}[Y]} \leq \quad \operatorname{Cov}(X, Y) & \leq \sqrt{\operatorname{Var}[X] \mathbb{V a r}[Y]}
\end{aligned}
$$


a covariância de $X$ e $Y$ é menor e igual à raiz quadrada positiva da multiplicação das suas variâncias.

\section{C.6 Funções Geratrizes}

As funções geratrizes são um método simbólico. É muito utilizado para tratar sequências de números. A função generatriz tem a seguinte forma:

$$
G(z)=g_{o}+g_{1} s+g_{2} s^{2}+\ldots=\sum_{i \geq 0} g_{i} s^{i}
$$

Disse-se que $G(s)$ é a função generatriz para a sequência $\left\langle g_{0}, g_{1}, g_{2}, \ldots.\right\rangle$, o qual também é denominado como $\left\langle g_{i}\right\rangle$.

\section{C.6.1 Funções Geratrizes de Probabilidade}

Seja $X$ uma v.a. que toma somente valores de inteiros no negativos, a função generatriz de probabilidade ou FGP de $X$ é:

$$
G_{X}(s)=\sum_{i \geq 0} P(X=i) s^{i}
$$

Essa serie de potencias em $s$ contem toda a informação acerca da v.a.d. $X$.

Os coeficientes de $G_{X}(s)$ são no negativos y sua somatória é 1 , ou seja:

$$
G_{X}(1)=1
$$

A vantagem das funções geratrizes de probabilidade é que normalmente simplificam o cálculo de valor esperados y variância. O valor esperado está definido como:

$$
\begin{aligned}
\mathbb{E}[X] & =\sum_{i \geq 0} i P(X=i) \\
& =\sum_{i \geq 0} P(X=i) i .\left.s^{i-1}\right|_{s=1} \\
& =G_{X}^{\prime}(1)
\end{aligned}
$$

Ou seja, é a derivada da FGP em relação a $s$ e atribui-se $s=1$.

A variância está definido como:

$$
\begin{aligned}
\mathbb{E}\left[X^{2}\right] & =\sum_{i \geq 0} i^{2} P(X=i) \\
& =\left.\sum_{i \geq 0} P(X=i)\left(i(i-1) s^{i-2}+i s^{i-1}\right)\right|_{s=1} \\
& =G_{X}^{\prime \prime}(1)+G_{X}^{\prime}(1)
\end{aligned}
$$

Portanto:

$$
\operatorname{Var}(X)=G_{X}^{\prime \prime}(1)+G_{X}^{\prime}(1)+G_{X}^{\prime}(1)^{2}
$$

As equações C.13 e C.15 indicam que podemos calcular o valor esperado e a variância a partir das derivadas $G_{X}^{\prime}(1)$ e $G_{X}^{\prime \prime}(1)$. 


\section{Referências Bibliográficas}

[AG] D. F. Aranha e C. P. L. Gouvêa. RELIC is an Efficient LIbrary for Cryptography. http://code.google.com/p/relic-toolkit/. 51

[BDF98] Dan Boneh, Glenn Durfee e Yair Frankel. An attack on rsa given a small fraction of the private key bits. Em Advances in CryptologyâASIACRYPTâ98, páginas 25-34. Springer, 1998. 1,20

[BE03] H. Bar-El. Introduction to side channel attacks. White Paper. Discretix Technologies Ltd, 2003. 12

[Bin] Binomial theorem. Disponível em http://en.wikipedia.org/wiki/Binomial_theorem. 69

[CC98] Geoffrey Mallin Clarke e Dennis Cooke. A basic course in statistics. Arnold New York, 1998. 69,77

[Con] Scott Contini. General purpose factoring records. Disponível em http://www. crypto-world.com/FactorRecords.html. 1, 10

[Cop97] D. Coppersmith. Small solutions to polynomial equations, and low exponent rsa vulnerabilities. Journal of Cryptology, 10(4):233-260, 1997. 1

[Cor] Compaq Computer Corporation. Cryptography using compaq multi-prime technology in a parallel processing environment. Disponível em ftp://ftp.compaq.com/pub/solutions/ CompaqMultiPrimeWP.pdf. 10

[DH76] Whitfield Diffie e Martin E. Hellman. New directions in cryptography, 1976. 3

[Feo] Paulo Feofiloff. Introdução informal à complexidade de problemas. 1

[GNU] Gnu lesser general public license. http://www.gnu.org/licenses/lgpl.html. 51

[HM08] Mathias Herrmann e Alexander May. Solving linear equations modulo divisors: On factoring given any bits. Em Advances in Cryptology-ASIACRYPT 2008, páginas 406424. Springer, 2008. 1

[Hoo05] David Hook. Beginning cryptography and PKI in Java. 2005. 3

[HS09] Nadia Heninger e Hovav Shacham. Reconstructing rsa private keys from random key bits, 2009. 1, 13, 20, 23, 25, 38, 42, 49, 53, 62

$\left[\mathrm{HSH}^{+} 09\right]$ J.A. Halderman, S.D. Schoen, N. Heninger, W. Clarkson, W. Paul, J.A. Calandrino, A.J. Feldman, J. Appelbaum e E.W. Felten. Lest we remember: cold-boot attacks on encryption keys. Communications of the ACM, 52(5):91-98, 2009. 12, 13, 14

[JK03] J. Jonsson e B. Kaliski. Public-key cryptography standards (pkcs)\# 1: Rsa cryptography specifications version 2.1. Relatório técnico, RFC 3447, February, 2003. 4, 6, 10 
[KKY12] Jun Kogure, Noboru Kunihiro e Hirosuke Yamamoto. Generalized security analysis of the random key bits leakage attack. Em Information Security Applications, páginas 13-27. Springer, 2012. 38, 42, 60

[KL08] J. Katz e Y. Lindell. Introduction to modern cryptography. Chapman \& Hall/CRC cryptography and network security. Chapman \& Hall/CRC, 2008. 64, 68

[Lab91a] RSA Labs. Public-key cryptography standards (pkcs), 1991. Disponível em http://www. rsa.com/rsalabs/node.asp?id=2124. 10

[Lab91b] RSA Labs. Syslinux project, 1991. Disponível em http://www.syslinux.org/wiki/index. php/The_Syslinux_Project. 13

[Len01] Arjen K Lenstra. Unbelievable security matching aes security using public key systems. Em Advances in CryptologyâASIACRYPT 2001, páginas 67-86. Springer, 2001. 9, 10

[Mau92] U.M. Maurer. Factoring with an oracle. Em Proceedings of the 11th annual international conference on Theory and application of cryptographic techniques, páginas 429436. Springer-Verlag, 1992. 1

[MSSG10] S. Maitra, S. Sarkar e S. Sen Gupta. Publishing upper half of rsa decryption exponent. Advances in Information and Computer Security, páginas 25-39, 2010. 20, 38

[Pai03] Cesar Alison Monteiro Paixao. Implementaç ao e análise comparativa de variaç oes do criptossistema RSA. Tese de Doutorado, Masterâs thesis, Inst. de Matemática e Estatıstica, Univ. de Sao Paulo, 2003. 1

[Pea] Pearson product moment correlation coefficient. Disponível em http://en.wikipedia.org/ wiki/Pearson_product-moment_correlation_coefficient. 77

[Per05] Colin Percival. Cache missing for fun and profit. Em Proc. of BSDCan 2005, 2005. 17

[QC82] J.J. Quisquater e C. Couvreur. Fast decipherment algorithm for rsa public-key cryptosystem. Electronics letters, 18(21):905-907, 1982. 5

[Rhe94] M.Y. Rhee. Cryptography and secure communications. McGraw-Hill series on computer communications. McGraw-Hill, 1994. 63

[RSA78] R.L. Rivest, A. Shamir e L. Adleman. A method for obtaining digital signatures and public-key cryptosystems. Communications of the ACM, 21(2):120-126, 1978. 1, 4

[Sko02] S. Skorobogatov. Low temperature data remanence in static ram. University of Cambridge Computer Laborary Technical Report, 536, 2002. 12

[SS98] Adi Shamir e Nicko Van Someren. Playing hide and seek with stored keys. Em Lecture Notes in Computer Science, páginas 118-124, 1998. 14

[Ter00] R. Terada. Segurança de dados: criptografia em redes de computador. Edgard Blucher, 2000. $9,12,66$

[TV] Routo Terada e Reynaldo C. Villena. Factoring the multi-prime modulus n with random bits. Disponível em http://www.ime.usp.br/ reynaldo/sem/FactoringN.pdf. 32, 62

[Zim] Paul Zimmermann. Integer factoring records. Disponível em http://www.loria.fr/ zimmerma/records/factor.html. 10 


\section{Índice Remissivo}

Algoritmos de Euclides e Euclides Estendido, 64

Anel, 65

Ataques cold-boot, 12

Ataques side-channel, 11

Congruência, 63

Correção do PKCS, 9

Criptografia, 3

Criptografia de Chave Pùblica, 3

divisibilidade, 63

Divisibilidade e Congruências, 63

exemplo QC-RSA, 7

exemplo RSA, 5

exemplo RSA multi-primo, 5

Função Euler, 65

Grupo, 65

Grupo e Anel, 65

Inversa Multiplicativa Módulo N, 64

PKCS, 10

PKCS \#1, 10

Probabilidade, 71

QC-RSA, 5

RSA, 4

Segurança do RSA, 9

Teorema Chinês do Resto - TCR, 67 